Integração numérica de sistemas

não lineares semi-implícitos

via teoria de controle geométrico

Celso Bernardo da Nóbrega de Freitas

\author{
DissertaÇÃO APRESENTADA \\ AO \\ Instituto DE MATEMÁticA E EstatísticA \\ DA \\ Universidade DE SÃo PAUlo \\ PARA \\ OBTENÇÃO DO TÍTULO \\ $\mathrm{DE}$ \\ Mestre em CiÊnCIAS
}

Programa: Matemática Aplicada

Orientador: Prof. Dr. Paulo Sérgio Pereira da Silva 


\title{
Integração numérica de sistemas não lineares semi-implícitos via teoria de controle geométrico
}

\author{
Esta tese contém as correções e alterações \\ sugeridas pela Comissão Julgadora durante a defesa \\ realizada por Celso Bernardo da Nóbrega de Freitas em 04/11/2011. \\ O original encontra-se disponível no Instituto de \\ Matemática e Estatística da Universidade de São Paulo.
}

Comissão Julgadora:

- Prof. Dr. Paulo Sérgio Pereira da Silva (orientador) - Poli-USP

- Prof. Dr. Pedro Aladar Tonelli - IME-USP

- Prof. Dr. Carlos Corrêa Filho - FEI-SP 


\section{Resumo}

Neste trabalho aprimorou-se um método para aproximar soluções de uma classe de equações diferenciais algébricas (DAEs), conhecida como sistemas semi-implícitos quadrados. O método, chamado aqui de MII, fundamenta-se na teoria geométrica de desacoplamento para sistemas não lineares, aliada a técnicas eficientes de análise numérica. Ele usa uma estratégia mista com cálculos simbólicos e numéricos para construir um sistema explícito, cujas soluções convergem exponencialmente para as soluções do sistema implícito original. Duas versões do método são apresentadas. Com a primeira, chamada de MIIcond, procura-se obter matrizes numericamente estáveis, através de balanceamentos. E a segunda, MIIproj, aproveita uma interpretação geométrica para o campo vetorial obtido. As implementações foram desenvolvidas em Matlab/simulink com o pacote de computação simbólica. Através dos benchmarks, realizando inclusive comparações com outros métodos atualmente disponíveis, constatou-se que o MIIcond foi inviável em alguns casos, devido ao tempo de processamento muito extenso. Por outro lado, o MIIproj mostrou-se uma boa alternativa para esta classe de problemas, em especial para sistemas de alto índex.

Palavras-chave: teoria de controle geométrico, DAEs, sistemas semi-implícitos quadrados, integração numérica. 


\section{Abstract}

This work improves a method to approximate solutions for a class of differential algebraic equations (DAEs), known as systems semi-implicit square. The method, called here MII, is based on geometric theory of decoupling for nonlinear systems combined with efficient techniques numerical analysis. It uses an algorithum that mixes symbolic and numerical calculations to build an explicit system, whose solutions converge exponentially to solutions of the original implicit system. Two versions of the method are given. The first one is called MIIcond, trying to obtain numerically stable matrices through balancing. The second one is the MIIproj, taking advantage of a geometric interpretation of the vector field there obtained. The implementations were developed in Matlab/Simulink with the symbolic toolbox. Through benchmarks, including performing comparisons with other methods currently available, it was found that the MIIcond was not feasible in some cases, due to processing time too long. On the other hand, the MIIproj presented itself as good alternative to this class of problems, especially for systems of high index.

Keywords: geometric control theory, DAEs, semi-implicit square systems, numerical integration. 


\section{Sumário}

Lista de Figuras $\quad$ vii

$\begin{array}{lc}\text { Lista de Tabelas } & \text { ix }\end{array}$

1 Introdução $\quad 1$

1.1 Considerações Preliminares . . . . . . . . . . . . . . . . . . . . . . . 3

1.2 Notações . . . . . . . . . . . . . . . . . . . . . . . . . . . . . . . 6

1.3 Histórico e Objetivos . . . . . . . . . . . . . . . . . . . . . . 7

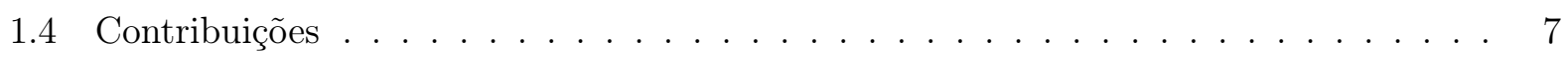

1.5 Organização do Trabalho . . . . . . . . . . . . . . . . . . 7

2 Sistema Explícito Teórico $\quad 9$

2.1 Teoria do Desacoplamento . . . . . . . . . . . . . . . . . . . . 9

2.2 Existência de Coordenadas Complementares . . . . . . . . . . . . . . . . . 17

2.3 Método por Realimentação de Estado Estática - MI . . . . . . . . . . . . . . . . . 18

2.4 Sistemas Explícitos versus Semi-Implícitos . . . . . . . . . . . . . . . . . 19

2.5 Método por Injeção de Saída Generalizada - MII . . . . . . . . . . . . . . . . . . 23

2.6 Teoremas de Proximidade . . . . . . . . . . . . . . . . . . . . . . 24

3 Sistema Explícito Numérico $\quad 31$

3.1 Coordenadas por Derivação de Saída . . . . . . . . . . . . . . . . . . . . 31

3.2 Complemento Ortogonal . . . . . . . . . . . . . . . . . . . . 33

3.3 Coordenadas Complementares via Complemento Ortogonal - $\mathrm{MII}_{\mathrm{proj}} \ldots \ldots$. . . . . . 35

3.4 Coordenadas Complementares via Preservação de Condicionamento - MII $_{\text {cond }}$. . . . . 38

3.5 Sistemas Variantes no Tempo . . . . . . . . . . . . . . . . . . . . . . . . 40

3.6 Construção da Implementação . . . . . . . . . . . . . . . . . . . . . . . . . . . . 41

4 Integração Numérica $\quad 45$

4.1 Estrutura dos Problemas e Características da Simulação . . . . . . . . . . . . . . 45

4.2 Problema MatrizCond . . . . . . . . . . . . . . . . . . . . . . 49

4.3 Problema Pêndulo . . . . . . . . . . . . . . . . . . . . . . . 52

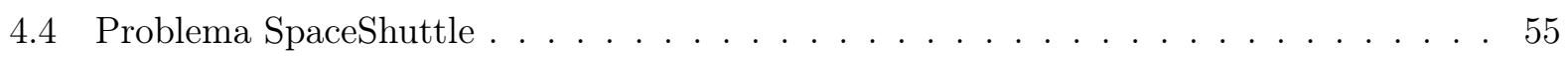

4.5 Problema Chemakzo . . . . . . . . . . . . . . . . . . . 57

4.6 Problema Andrews . . . . . . . . . . . . . . . . . . . . . . 59

4.7 Problema TransAmp . . . . . . . . . . . . . . . . . . . . . . . 61

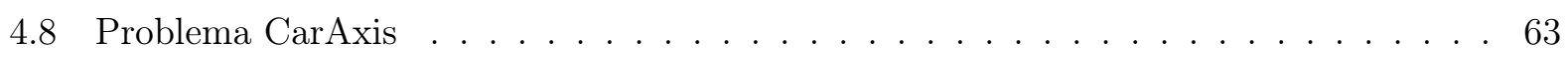


4.9 Conclusões sobre as Implementações . . . . . . . . . . . . . . . . . . . 66

5 Conclusões $\quad 67$

5.1 Sugestões para Pesquisas Futuras . . . . . . . . . . . . . . . 68

6 Apêndice $\quad \mathbf{7 1}$

6.1 Código-Fonte Construção da Implementação . . . . . . . . . . . . . . . . . . . . . . . 71

6.2 Código-Fonte MatrizCond ． . . . . . . . . . . . . . . . . . . 78

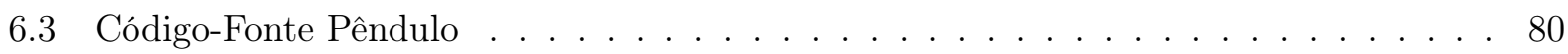

6.4 Código-Fonte SpaceShuttle . . . . . . . . . . . . . . . . . . . . . . 81

6.5 Código-Fonte Chemakzo . . . . . . . . . . . . . . . . . . . . . . . 84

6.6 Código-Fonte Andrews . . . . . . . . . . . . . . . . . . . . . 86

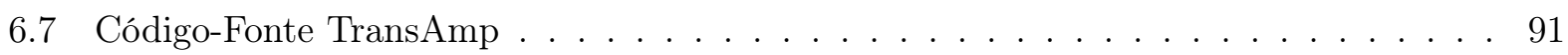

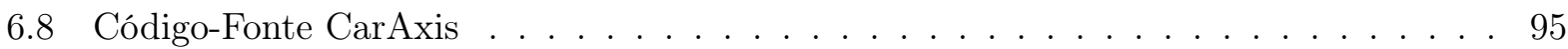

$\begin{array}{ll}\text { Referências Bibliográficas } & 99\end{array}$ 


\section{Lista de Figuras}

1.1 Soluções do Sistema Implícito evoluindo na variedade $\Gamma \ldots \ldots$. . . . . . . . . . . . 2

1.2 A trajetória de $(\bar{x}(t), \bar{u}(t))$ aproximando $(x(t), u(t))$ permanece perto de $\Gamma \ldots \ldots$

1.3 Esquema com estratégia para aproximação das soluções do Sistema Implícito. . . . . 5

2.1 Exemplo de comportamento indesejado para o erro $y^{(0)}$ utilizando o MI. . . . . . . . 22

3.1 Interpretação geométrica das componentes do campo vetorial $\tau_{x}$ do $\mathrm{MII}_{\text {proj. }}$. . . . . 36

3.2 Diagrama de simulação, padrão Matlab. . . . . . . . . . . . . . . . . . . . . 42 


\section{Lista de Tabelas}

4.1 Problemas Simulados: abreviatura, nome, área e referência . . . . . . . . . . . . 48

4.2 Problema MatrizCond: MII, rtol $=$ atol $=h_{0}=10^{-4} \ldots \ldots \ldots \ldots \ldots$

4.3 Problema MatrizCond: MII, condicionamento . . . . . . . . . . . . . . . 50

4.4 Problema Pêndulo: MII, rtol $=$ atol $=h_{0}=10^{-4} \ldots \ldots \ldots \ldots \ldots \ldots$

4.5 Problema Pêndulo: MII, rtol $=$ atol $=h_{0}=10^{-6} \ldots \ldots \ldots \ldots \ldots \ldots$

4.6 Problema SpaceShuttle: MII, rtol $=$ atol $=h_{0}=10^{-4} \ldots \ldots \ldots \ldots \ldots$

4.7 Problema SpaceShuttle: $\mathrm{MII}_{\mathrm{proj}}$, rtol $=$ atol $=h_{0}$, refinamento de passo $\ldots \ldots \ldots 5$

4.8 Problema Chemakzo: MII, rtol $=$ atol $=h_{0}=10^{-10} \ldots \ldots \ldots \ldots \ldots$

4.9 Problema Chemakzo: Outros Solveres . . . . . . . . . . . . . . . . . 58

4.10 Problema Chemakzo: $\mathrm{MII}_{\text {proj }}$, rtol $=$ atol $=h_{0}=10^{-10}$, ode113 $\ldots \ldots \ldots$. . . 58

4.11 Problema Andrews: MII, rtol $=$ atol $=h_{0}=10^{-4} \ldots \ldots \ldots \ldots \ldots$. . . . . 59

4.12 Problema Andrews: MII, rtol $=$ atol $=h_{0}=10^{-7} \ldots \ldots \ldots \ldots \ldots$. . . . . 59

4.13 Problema Andrews: $\mathrm{MII}_{\mathrm{proj}}, \mathrm{rtol}=$ atol $=h_{0}$, refinamento de passo $\ldots \ldots \ldots$. . . . 59

4.14 Problema Andrews: Outros Solveres . . . . . . . . . . . . . . . . . 60

4.15 Problema Transamp: MII, rtol $=$ atol $=10^{-4}, h_{0}=10^{-6} \ldots \ldots \ldots \ldots$. . . . . 61

4.16 Problema Transamp: MII, rtol $=$ atol $=10^{-7}, h_{0}=10^{-9} \ldots \ldots \ldots \ldots$. . . . . 62

4.17 Problema TransAmp: Outros Solveres . . . . . . . . . . . . . . . . . . . 62

4.18 Problema CarAxis: MII, rtol $=$ atol $=h_{0}=10^{-4} \ldots \ldots \ldots \ldots$. . . . . . 63

4.19 Problema CarAxis: MII, rtol $=$ atol $=h_{0}=10^{-7} \ldots \ldots \ldots \ldots$

4.20 Problema CarAxis: MII, rtol $=$ atol $=h_{0}=10^{-10} \ldots \ldots \ldots \ldots \ldots$. . . . . . 64

4.21 Problema CarAxis: Outros Solveres . . . . . . . . . . . . . . . . . . . . 64

4.22 Problema CarAxis: $\mathrm{MII}_{\mathrm{proj}}, \mathrm{rtol}=$ atol $=h_{0}$, ode113 $\ldots \ldots \ldots \ldots \ldots$ 


\section{Capítulo 1}

\section{Introdução}

Equações diferenciais ordinárias surgem na modelagem de sistemas em muitas áreas da ciência e tecnologia. No entanto, muitos modelos matemáticos em engenharia mecânica, elétrica, química, etc. [Cam82, Dai89, Cam90, KD99, Sto98, HW96, HLR89, Sch94] são construídos naturalmente como equações diferenciais ordinárias com restrições algébricas. Tal formulação, conhecida como equação diferencial algébrica (DAE), pode ser vista como uma generalização do conceito de equação diferenciais ordinária. As DAEs são sistemas da forma:

$$
F(\dot{x}(t), x(t), t)=0
$$

onde $x \in \mathbb{R}^{n}$ é um vetor de variáveis independentes e $F=\left(F_{1}, \ldots, F_{n}\right): \mathbb{R}^{2 n+1} \rightarrow \mathbb{R}^{n}$ é uma aplicação diferenciável que expressa a dinâmica do sistema.

O problema de integração numérica das DAEs é ainda um campo ativo de pesquisa e parte do estado da arte pode ser encontrado nos livros textos [BCP96, AP98]. A dificuldade de integração é em geral medida por um inteiro, denominado index, que avalia o número suficiente de derivadas da equação original para que se possa, ao menos teoricamente, explicitar a dinâmica.

Existem vários pacotes de integração numérica de DAEs, chamados aqui de solveres ${ }^{1}$, como por exemplo em [BMM06, BCP96, IM98, Cas03, SLV97, HW99], podendo ser encontrados também no sítio do projeto Test Set for Initial Value Problem Sovers ${ }^{2}$, [MM08].

Este trabalho explora um método para resolução numérica de uma classe importante de DAEs chamada de sistemas semi-implícitos, da forma:

$$
\left\{\begin{aligned}
\dot{x} & =f(x, u) \\
y & =h(x) \equiv 0 \\
x\left(t_{0}\right) & =x_{0}
\end{aligned}\right.
$$

Utilizando-se nomenclatura usual na teoria de controle, $x(t) \in \mathbb{R}^{n}$ representa o estado do sistema, para $t$ pertencente a um intervalo $I \doteq\left[t_{0}, t_{f}\right] \subset \mathbb{R}$, condição inicial $x_{0}$ tal que $h\left(x_{0}\right)=0$, $u \in \mathbb{R}^{m}$ representa sua entrada e $y \in \mathbb{R}^{l}$ sua saída.

A primeira parte do sistema (1.1a) corresponde à sua dinâmica, dada por uma equação diferencial ordinária na variável independente $t$, regida pelo campo vetorial diferenciável $f: \mathcal{A} \times \mathbb{R}^{m} \rightarrow \mathbb{R}^{n}$, onde $\mathcal{A}$ é um aberto de $\mathbb{R}^{n}$. A segunda parte (1.1b), com $h: \mathcal{A} \rightarrow \mathbb{R}^{l}$ sendo também uma função diferenciável, expressa as chamadas "restrições algébricas", impondo que o estado do sistema deve sempre pertencer à variedade $\{x \in \mathcal{A}: h(x)=0\}$.

Fixada uma função suave $u: I \rightarrow \mathbb{R}^{m}$ qualquer, diz-se que uma função diferenciável $x: I \rightarrow \mathbb{R}^{n}$ é uma solução do sistema se satisfizer ao mesmo tempo (1.1a), (1.1b) e (1.1c). Será visto que (1.1b) induz restrições no espaço $(x, u)$ fazendo com que $u$ não seja uma verdadeira entrada do sistema.

\footnotetext{
${ }^{1}$ Emprega-se neste trabalho o estrangeirismo comumente utilizado solver.

${ }^{2}$ http://pitagora.dm.uniba.it/ testset/
} 
Assim, como as entradas que geram soluções do sistema também não são arbitrárias, justifica-se o uso do termo pseudo-estado e pseudo-entrada para essas funções. Por esse motivo, é admissível o abuso de nomenclatura ao dizer que o par $(x(t), u(t))$ é uma solução de (1.1).

Será mostrado no decorrer do trabalho que existe uma subvariedade $\Gamma \subset \mathcal{A} \times \mathbb{R}^{m}$ na qual evoluem as soluções de (1.1).

Denote-se por $\varphi\left(t, x_{0}, u(t)\right)$ a solução de (1.1) no instante $t$, partindo-se de $x_{0}$ com entrada $u$. Para uma mesma condição inicial $x_{0}$ podem existir diferentes entradas $u(t)$ e $\tilde{u}(t)$ tais que $\varphi\left(t, x_{0}, u(t)\right)$ e $\varphi\left(t, x_{0}, \tilde{u}(t)\right)$ são soluções de (1.1), como ilustrado na Figura 1.1. Neste caso, o sistema não é completamente determinado e há alguma liberdade de escolha em $u(t)$.

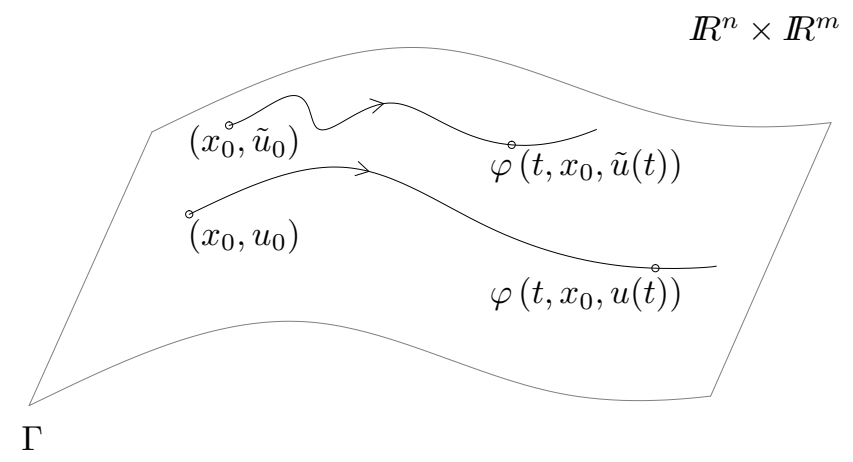

Figura 1.1: Soluções do Sistema Implícito evoluindo na variedade $\Gamma$.

Contudo, o presente trabalho lidará somente com os sistemas ditos quadrados, nos quais a dimensão da entrada é a mesma da saída, i.e., com $l=m$, implicando determinação completa das soluções como será visto adiante.

Por vezes aqui, sistemas semi-implícitos quadrados serão chamados simplesmente de sistemas implícitos, quando não prejudicar o entendimento.

O método objeto deste trabalho denomina-se MII. Ele é baseado em teoria geométrica de desacoplamento para sistemas não lineares, aliada a métodos numéricos eficientes, permitindo construir, através de um algoritmo simbólico/numérico, um campo vetorial $\tau(x, u)$, de forma que a aproximação das soluções de (1.1) pode ser feita através da equação diferencial:

$$
\left(\begin{array}{c}
\dot{x} \\
\dot{u}
\end{array}\right)=\tau(x, u) ; \quad x\left(t_{0}\right)=x_{0} ; \quad u\left(t_{0}\right)=u_{0}
$$

onde $u_{0} \in \mathbb{R}^{m}$ é completamente determinado, podendo ser visto como a única escolha para a qual o vetor $f\left(x_{0}, u_{0}\right)$ pertence ao espaço tangente à superfície que anula identicamente as saídas no ponto $x_{0}$. E, $\tau: \mathcal{A} \times \mathbb{R}^{m} \rightarrow \mathbb{R}^{n \times m}$ é um campo vetorial suave explícito, de maneira que a solução $(x(t), u(t))$ de (1.2) também é solução de (1.1).

Observa-se que, diferentemente das técnicas empregadas pelos demais solveres em [MM08], é possível aplicar diretamente os métodos usuais para integração numérica de equações diferenciais ordinárias ao Sistema Explícito (1.2).

Contudo, em geral, métodos numéricos geram erros em relação à solução analítica. Ou ainda, devido à precisão finita, pode ocorrer que a condição inicial $\left(\bar{x}_{0}, \bar{u}_{0}\right)$ não pertença exatamente a $\Gamma$, estando sim à uma distância $\varepsilon>0$ pequena de $\Gamma$. Torna-se necessário construir um campo $\tau(x, u)$ robusto, no sentido de que se $(\bar{x}(t), \bar{u}(t))$ é uma solução de (1.2) definida para $t$ num intervalo $I_{1}$ compacto, partindo da condição inicial $\left(\bar{x}_{0}, \bar{u}_{0}\right)$, então $(\bar{x}(t), \bar{u}(t))$ ficará próxima de soluções do 
Sistema Implícito; de forma que $\|h(\bar{x}(t))\|$ ainda será pequeno para todo $t \in I_{1}$, guardadas certas condições. A Figura 1.2. indica o conceito.

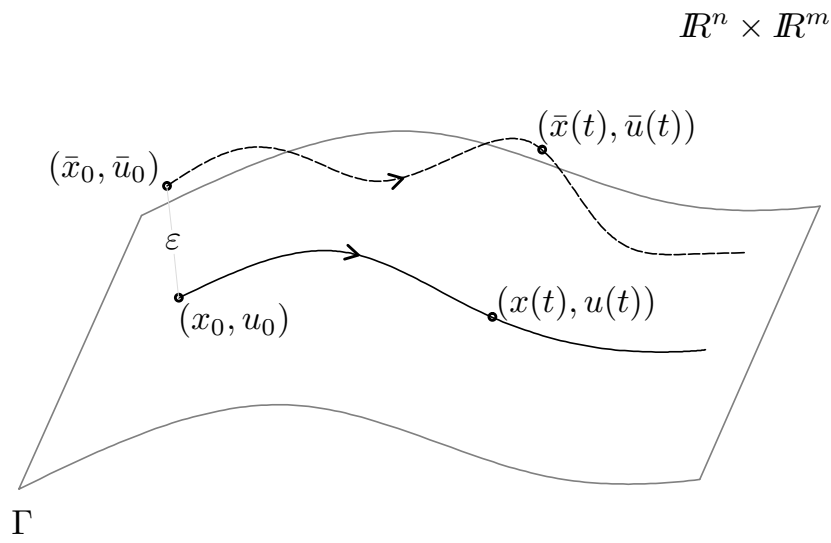

Figura 1.2: A trajetória de $(\bar{x}(t), \bar{u}(t))$ aproximando $(x(t), u(t))$ permanece perto de $\Gamma$.

\subsection{Considerações Preliminares}

Esta seção destina-se apenas a fornecer uma primeira ideia sobre o método, com o intuito de facilitar o posterior entendimento do papel desempenhado por cada tópico da teoria.

A estratégia utilizada pelo MII para a aproximação das solução do sistema (1.1) consiste nas etapas:

\section{Etapa 1: Transformação do Sistema Implícito Original em um Sistema Explícito Teórico}

O primeiro resultado importante para esta etapa é a construção do conjunto $\Gamma$ no qual evoluem as soluções do Sistema Implícito. Ora, se $(x(t), u(t))$, definida num intervalo $I_{1} \subset \mathbb{R}$, é uma solução para o (1.1), em particular $y=h(x(t)) \equiv 0, \forall t \in I_{1}$. Mais ainda, as derivadas de $y=h(x(t))$ em relação à $t$ de todas as ordens também serão nulas, i.e., $\forall k>0, \frac{d}{d t^{k}}(y) \equiv 0$. Para $k=1$, por exemplo, a derivada da primeira componente da saída $y_{1}^{(0)} \doteq y_{1}$ é dada por $\frac{d}{d t}\left(y_{1}\right)=\frac{\partial h_{1}}{\partial x} \dot{x}=\frac{\partial h_{1}}{\partial x} f(x, u) \doteq y_{1}^{(1)}$. Observando que se a derivada parcial de $y_{1}^{(1)}$ em relação à entrada $u$ for não nula numa vizinhança $\mathcal{V}$ da condição inicial $\left(x_{0}, u_{0}\right)$, pode-se dizer que $y_{1}^{(1)}=y_{1}^{(1)}(x, u)$ depende explicitamente de $u$ em $\mathcal{V}$, caso contrário, diz-se que $y_{1}^{(1)}=y_{1}^{(1)}(x)$ não depende explicitamente de $u$.

A derivada de ordem $k$ da i-ésima componente da saída pode ser construída iterativamente, denotando-se $y_{i}^{(k+1)} \doteq \frac{d}{d t}\left(y_{i}^{(k)}\right)$. Portanto, pode-se derivar sucessivamente cada uma das componentes de saída, $y_{i}=h_{i}(x)$, para $i \in\{1, \ldots, m\}$, até que $y_{i}^{\left(\rho_{i}\right)}$ dependa explicitamente da $u$ em $\mathcal{V}_{i}$ (vide Observação 2.15). Para fixar ideias, assume-se que a vizinhança $\mathcal{V}_{i}$ na qual $y_{i}^{\left(\rho_{i}\right)}$ depende explicitamente de $u$ é igual a $\mathcal{A}$ para todo $i \in\{1, \ldots, m\}$.

O inteiro $\rho \doteq \sum_{i=1}^{m} \rho_{i}$ é chamado de grau relativo do sistema. O valor máximo entre as constantes $\left\{\rho_{1}, \ldots, \rho_{m}\right\}$ recebe o nome de index de uma DAE. ${ }^{3}$

Originam-se então $\rho$ funções suaves, denotadas por $Y^{(\rho-1)}: \mathcal{A} \rightarrow \mathbb{R}^{\rho}$, escritas como:

$$
Y^{(\rho-1)}(x)=\left(y_{1}^{(0)}(x), \ldots, y_{1}^{\left(\rho_{1}-1\right)}(x), \ldots, y_{m}^{(0)}(x), \ldots, y_{m}^{\left(\rho_{m}-1\right)}(x)\right)
$$

\footnotetext{
${ }^{3}$ Como sistemas explícitos podem ser vistos como DAEs de index zero e o MII obtém uma representação explícita para sistemas semi-implícitos quadrados, o MII pertence à classe dos métodos de redução de index.
} 
Definem-se também $m$ funções suaves $y^{(\rho)}: \mathcal{A} \times \mathbb{R}^{m} \rightarrow \mathbb{R}^{m}$, dadas por:

$$
y^{(\rho)}(x, u)=\left(y_{1}^{\left(\rho_{1}\right)}(x, u), \ldots, \ldots, y_{m}^{\left(\rho_{m}\right)}(x, u)\right)
$$

Vetorialmente, indicar-se-á $Y^{(\rho)}(x, u)=\left(Y^{(\rho-1)}(x), y^{(\rho-1)}(x, u)\right)$.

Como $h(x, u) \equiv 0$ implica $Y^{(\rho)}(x, u)=0$, pois ambas expressões impõem que a saída do Sistema Implícito seja identicamente nula, vale que $\Gamma \doteq\left\{(x, u) \in \mathcal{A} \times \mathbb{R}^{m}: Y^{(\rho)}(x, u)=0\right\}$. É claro que a norma $\left\|Y^{(\rho)}(\bar{x}, \bar{u})\right\|$ é uma medida da distância do ponto $(\bar{x}, \bar{u})$ em relação à variedade $\Gamma$.

Cabe frisar do ponto de vista computacional que a construção da função $Y^{(\rho)}(x, u)$ será feita pelo Algoritmo (3.2), implementado em Matlab utilizando cálculos simbólicos.

O segundo resultado importante para a definição do método, apresentado no Corolário 2.20, diz que sob certas condições existe uma função suave $\hat{x}: \mathcal{A} \rightarrow \mathbb{R}^{n-\rho}$, aqui chamada de coordenadas complementares, tal que a aplicação $\Psi: \mathcal{A} \times \mathbb{R}^{m} \rightarrow \mathbb{R}^{n+m}$, dada por $\Psi(x, u)=\left(\hat{x}(x), Y^{(\rho)}(x, u)\right)$ é um difeomorfismo entre uma vizinhança $\mathcal{W}$ de $\left(x_{0}, u_{0}\right)$ e $\Psi(W)$. Como consequência, sobre as soluções $(x(t), u(t))$ do Sistema Implícito vale que $\Psi(x(t), u(t))=(\hat{x}(x), 0)$.

Portanto, pode-se expressar em coordenadas $\left(\hat{x}, Y^{(\rho)}\right)$ o Sistema Implícito (1.1) restrito à vizinhança $\mathcal{W}$, obtendo-se:

$$
\left\{\begin{aligned}
\dot{\hat{x}} & =\theta\left(\hat{x}, Y^{(\rho)}\right) \\
\dot{Y}^{(\rho)} & =-\gamma Y^{(\rho)} \\
\hat{x}\left(t_{0}\right) & =\hat{x}_{0} \\
Y^{(\rho)}\left(t_{0}\right) & =Y_{0}^{(\rho)}=0
\end{aligned}\right.
$$

onde:

- $\hat{x}_{0} \in \mathbb{R}^{\rho}$ é tal que $\Psi\left(x_{0}, u_{0}\right)=\left(\hat{x}_{0}, 0\right)$;

- $\theta\left(\hat{x}, Y^{(\rho)}\right)$ é a chamada dinâmica zero do sistema, consistindo na expressão de $\frac{\partial \hat{x}}{\partial x} f(x, u)$ em coordenadas $\left(\hat{x}, Y^{(\rho)}\right)$;

- $\gamma>0$ é uma constante, aqui denominada parâmetro de convergência

Note-se ainda que a solução analítica de $(1.5 \mathrm{~b})$ é dada por $Y^{(\rho)}(t)=e^{-\gamma(t-t 0)} Y_{0}^{(\rho)^{\pi^{0}}} \equiv 0$.

O MII é estabelecido, escrevendo novamente o sistema (1.5) nas coordenadas originais $(x, u)$ :

$$
\begin{cases}\left(\begin{array}{c}
\dot{x} \\
\dot{u}
\end{array}\right)= & \tau(x, u) \\
\text { Onde } & T(x, u) \tau(x, u)=\left(\begin{array}{c}
\frac{\partial \hat{x}}{\partial x}(f(x, u)) \\
-\gamma Y^{(\rho)}(x, u)
\end{array}\right) \\
\text { com } \quad & \left.T(x, u) \doteq \frac{\partial \Psi}{\partial(x, u)}\right|_{(x, u)}=\left(\begin{array}{cc}
\frac{\partial \hat{x}}{\partial x} & 0 \\
\frac{\partial Y^{(\rho-1)}}{\partial x} & 0 \\
\frac{\partial y^{(\rho)}}{\partial x} & \frac{\partial y^{(\rho)}}{\partial u}
\end{array}\right)\end{cases}
$$

cujas soluções ficam próximas das soluções do Sistema Implícito, desde que a distância entre a condição inicial dada e a variedade $\Gamma$ seja pequena. O motivo intuitivo para isso, formalizado na Seção 2.6, é que, mesmo para um erro inicial em relação à variedade $Y_{0}^{(\rho)}$ diferente de zero, a função $Y^{(\rho)}(t)=e^{-\gamma(t-t 0)} Y_{0}^{(\rho)}$ é uma função estritamente decrescente, cujo limite é zero. Entretanto, não 
se pode integrar numericamente (1.6) por não se ter a expressão para o campo $\tau(x, u)$. Isto pois se sabe que existe, mas ainda não se fixou uma escolha de coordenadas complementares $\hat{x}(x)$, razão pela qual utilizou-se o adjetivo "teórico" para o sistema (1.6).

\section{Etapa 2: Transformação do Sistema Explícito Teórico em um Sistema Explícito Numérico}

De acordo com a Seção 3.2, é efetuada uma escolha numérica de coordenadas complementares $\hat{x}(x)$, possibilitando o cálculo de $\tau(x, u)$ pontualmente. Será utilizado o conceito de complemento ortogonal para gerar uma matriz $R(x)$ de dimensão $n \times(n-\rho)$, obtendo:

$$
T(x, u)=\left(\begin{array}{c|c}
R(x) & 0 \\
\frac{\partial Y^{(\rho-1)}}{\partial x} & 0 \\
\hline \frac{\partial y^{(\rho)}}{\partial x} & \frac{\partial y^{(\rho)}}{\partial u}
\end{array}\right)
$$

Onde $\frac{\partial \hat{x}(x)}{\partial x}$ foi imposto como sendo a matriz $R(x)$, resultando $T(x, u)$ não singular.

São expostas duas opções de coordenadas complementares, cujas ideias principais são: para a primeira, chamada de $\mathrm{MII}_{\mathrm{proj}}$, recalcular o complemento ortogonal em todo ponto, aproveitando a estrutura especial que a inversa de $T$ assume, como discutido na Seção 3.3; e a MII $_{\text {cond }}$, com coordenadas complementares como transformações lineares fixas por intervalos de tempo, trocadas quando o condicionamento dos blocos diagonais da matriz $T(x, u)$ ultrapassar uma tolerância, conforme Seção 3.4.

\section{Etapa 3: Integração Numérica do Sistema Explícito Numérico}

Utilizando métodos clássicos para EDOS, integrar numericamente o Sistema Explícito Numérico, processo este aqui indicado simplesmente por simulação. As simulações foram efetuadas com o Matlab/Simulink.

Importante esclarecer que a Etapa 2 também é feita durante a simulação, já que o campo $\tau(x, u)$ precisa ser avaliado somente sobre os pontos $\left(x_{t_{k}}, u_{t_{k}}\right)$, onde $t_{k}$, para $k \in\{0, \ldots, N\}$, são os instantes de tempo gerados pela discretização do intervalo de tempo na simulação.

O esquema abaixo resume as três etapas.

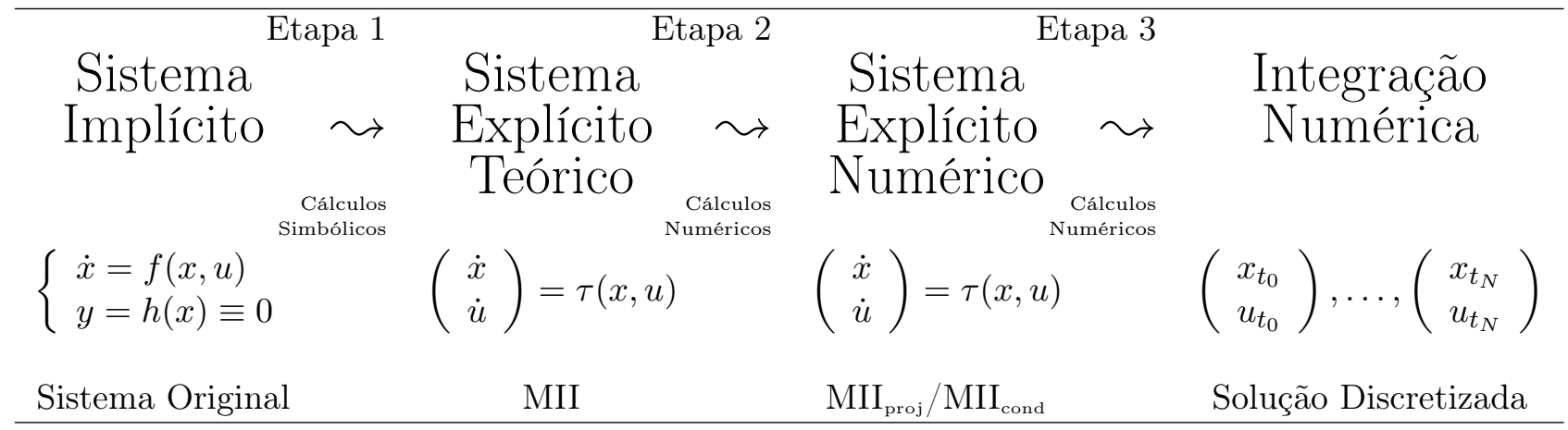

Figura 1.3: Esquema com estratégia para aproximação das soluções do Sistema Implícito. 


\subsection{Notações}

Descrevem-se aqui as principais notações e nomenclaturas:

- Todos os conjuntos abertos são tomados sobre topologia euclidiana.

- Para o ponto $x \in \mathbb{R}^{n},\|x\|$ indica a norma euclidiana, a menos de menção contrária.

- Para o ponto $x \in \mathbb{R}^{n},\|x\|_{\infty}$ indica a norma do máximo.

• Os nomes "função" e "aplicação" são empregados como sinônimos, mesmo quando o contradomínio possuir dimensão maior do que 1.

- O termo função suave será empregado para funções de classe $\mathcal{C}^{\infty}$.

- Se $x: I \rightarrow \mathbb{R}^{n}$ é uma função suave, com $I$ um intervalo de $\mathbb{R}$ e $t_{1} \in I, \dot{x}\left(t_{1}\right)$ indica $\left.\frac{d}{d t}(x)\right|_{t_{1}}$. Nos extremos do intervalo são tomadas as derivadas laterais apropriadas.

- Se $f: \mathcal{U} \rightarrow \mathbb{R}^{m}$ é uma função suave, com $\mathcal{U}$ uma vizinhança de $a$ em $\mathbb{R}^{n}$, a transformação linear $\left.\frac{\partial f}{\partial x}\right|_{a}: \mathbb{R}^{n} \rightarrow \mathbb{R}^{m}$ será identifica com a matriz a matriz jacobiana $\left(\left.\frac{\partial f_{i}}{\partial x_{j}}\right|_{a}\right)$ quando isto não prejudicar o entendimento.

- Para o campo vetorial suave $h: \mathcal{U} \rightarrow \mathbb{R}^{n}$, com $\mathcal{U}$ um aberto de $\mathbb{R}^{n}$, e $f: \mathcal{U} \rightarrow \mathbb{R}$ uma função suave, a Derivada de Lie $L_{h} f(x)$ é entendida como $\left.\frac{\partial f}{\partial h}\right|_{x}$, i.e., a derivada direcional de $f$ na direção $h(x)$ avaliada no ponto $x$.

- O espaço das matrizes reais de dimensão $n \times m$ é identificado com o espaço $\mathbb{R}^{n \times m}$.

- Para a matriz $M$, a notação $M_{n \times m}$ indica que $M$ tem dimensão $n \times m$.

- A matriz identidade de dimensão $n \times n$ é denotada por $I_{n \times n}$.

- A notação $M^{\top}$ indica a transposta de uma matriz $M$.

- Para vetores $u, v \in \mathbb{R}^{n},\langle u, v\rangle=u^{\top} v$ denota o produto interno usual.

- Se $M$ é uma matriz em $\mathbb{R}^{n \times m}$, a norma da matriz $\|M\|$ é igual a sup $\left\{\|M x\|, \forall x \in \mathbb{R}^{n}:\|x\|=1\right\}$.

- Se $V$ e $W$ são subespaços de $\mathbb{R}^{n}$ então $\mathbb{R}^{n}=V \oplus W$ significa que $\mathbb{R}^{n}$ é soma direta de $V$ e $W$.

- Se $u \in \mathbb{R}^{n}$ e $v \in \mathbb{R}^{m},(u, v)=\left(\begin{array}{c}u \\ v\end{array}\right)$ indica o vetor coluna de $\mathbb{R}^{n+m}$ formado pela justaposição de $u$ e $v$.

- Se $V$ é um espaço vetorial em $\mathbb{R}^{n}, V^{\perp}$ indica o complemento ortogonal de $V$.

- Se $V$ é subespaço vetorial de $\mathbb{R}^{n}$, a função $\operatorname{proj}_{V}: \mathbb{R}^{n} \rightarrow \mathbb{R}^{n}$ indica a projeção ortogonal sobre o espaço $V$, i.e., associa um vetor $x \in \mathbb{R}^{n} \equiv V \oplus V^{\perp}$ à sua projeção em $V$.

- Se $E=\mathbb{R}^{n} \times \mathbb{R}^{m}$, a função $\operatorname{proj}_{\mathbb{R}^{n}}: E \rightarrow E$ indica a projeção sobre o espaço $\mathbb{R}^{n}$. 


\subsection{Histórico e Objetivos}

O método MII começou a ser definido em [SW02], abordando principalmente a Etapa 1, através da teoria do desacoplamento.

O uso do complemento ortogonal foi proposto em [SDS05], possibilitando as etapas 2 e 3. Ainda em [SDS05], foi feito um experimento numérico mostrando vantagens do MII em relação ao método clássico (MI) por realimentação de estado estática, conforme [NS90].

Posteriormente, [Sou06] repassa a teoria para a Etapa 1, generalizando-a para campos vetoriais variantes no tempo e definindo uma técnica para busca de condições iniciais compatíveis com as restrições. Além disso, realizaram-se mais simulações ilustrativas.

Em seguida, implementa-se em [Fre07] um algoritmo utilizando cálculos simbólicos para a construção automática das simulações. Outras comparações entre o MI e MII também são feitas por meio de simulações, reforçando as qualidades do MII.

Finalmente, o principal objetivo do atual trabalho foi empregar ferramentas de análise numérica na Etapa 2, melhorando-o em termos de precisão e performance. Por fim, para testar os resultados obtidos, foram utilizados problemas difíceis (benchmarks) nas simulações.

\subsection{Contribuições}

As principais contribuições deste trabalho são:

- Maior detalhamento dos teoremas e provas que discutem a proximidade entre as soluções do MII e do Sistema Implícito na Seção 2.6. Em especial, uma reformulação destes resultados que consistem no Teorema 2.27.

- Interpretação geométrica e justificativa da escolha de coordenadas complementares MII $_{\text {proj }}$, presente na Seção 3.3.

- Definição de coordenadas complementares por preservação de condicionamento MII $_{\text {cond }}$, registrada na Seção 3.4.

- Por à prova os resultados obtidos através de simulações de benchmarks, análise dos resultados e comparação com outros métodos (Capítulo 4).

\subsection{Organização do Trabalho}

O segundo capítulo corresponde à Etapa 1 descrita na Seção 1.1. Nele, estudam-se conceitos de controle geométrico como a teoria do desacoplamento, permitindo a construção das coordenadas $Y^{(\rho)}(x, u)$ e provando a existência de coordenadas complementares $\hat{x}(x)$. Na última seção incluem-se os teoremas que analisam a proximidade entre as soluções do MII e o Sistema Implícito.

A Etapa 2 é assunto do terceiro capítulo, exibindo-se duas possibilidades numéricas para escolha de coordenadas complementares. Além disso, são exibidos os algoritmos necessários na construção das simulações para MII $_{\text {proj }}$ e MII $_{\text {cond }}$.

O quarto capítulo traz alguns exemplos ilustrativos da teoria, abrangendo a Etapa 3. Simulações com benchmarks são efetuadas, bem como a análise dos resultados e comparação com outros métodos.

No apêndice constam os códigos-fonte utilizados para as simulações. 


\section{Capítulo 2}

\section{Sistema Explícito Teórico}

Este capítulo registra sucintamente a teoria necessária para a obtenção de um sistema explícito teórico de equações diferenciais, a partir de um sistema quadrado semi-implícito, utilizando-se para isso de controle geométrico.

Com a teoria de desacoplamento, avalia-se sob quais condições pode-se obter uma transformação para um sistema de controle explícito, a fim de que cada uma das componentes da saída seja afetada por apenas uma das componentes de entrada. Discute-se em seguida a existência de coordenadas complementares que, diferentemente do pseudo-estado, representarão a evolução das soluções "vistas de dentro" do conjunto que anula identificamente as saídas. Inclui-se então a estrutura do método MI por realimentação de estado estática utilizando essas ferramentas, passando pela relação entre sistemas explícitos e semi-implícitos com essa abordagem, bem como algumas questões sobre a sua aplicação.

Finalmente, o método MII objeto deste trabalho é apresentado, baseando-se em uma transformação de coordenadas sobre o espaço de estados e de entradas, realimentação de estado dinâmica e uma injeção generalizada da saída. Para formalização das ideias, estudam-se teoremas que discutem como as soluções desse novo sistema aproximam as soluções do sistema implícito original.

\subsection{Teoria do Desacoplamento}

Por um momento, será esquecido o fato de que esta dissertação estuda sistemas implícitos (1.1), para estudar certas propriedades de sistemas explícitos, onde a restrição algébrica (1.1b) está ausente.

Seja o seguinte sistema não linear afim explícito, dado por sua representação de estado:

$$
\left\{\begin{array}{l}
\dot{x}=f(x)+\sum_{i=1}^{m} g_{i}(x) u_{i}(x) \\
y=h(x)
\end{array}\right.
$$

Onde:

$x$ representa o estado do sistema, definido num aberto $\mathcal{A} \subset \mathbb{R}^{n}$.

$u=\left(u_{1}, \ldots, u_{m}\right): \mathcal{A} \rightarrow \mathbb{R}^{m}$ uma função de entrada arbitrária para o sistema.

$y \in \mathbb{R}^{m}$ é o espaço de saída.

$f: \mathcal{A} \rightarrow \mathbb{R}^{n}$ campo vetorial que descreve a dinâmica do sistema para entrada nula.

$g_{i}: \mathcal{A} \rightarrow \mathbb{R}^{n}$ campo vetorial que descreve como a entrada $u_{i}$ modifica a dinâmica do sistema.

$h: \mathcal{A} \rightarrow \mathbb{R}^{m}$ função que associa um estado do sistema à sua saída.

$t \in I$ é a variável de tempo, com $I=\left[t_{0}, t_{f}\right] \in \mathbb{R}$ um intervalo no qual estarão definidas as soluções do sistema para uma determinada condição inicial $x_{0} \in \mathcal{A}$ no instante $t_{0}$. Sem perda de generalidade, fixa-se $t_{0}=0$, pois o sistema é invariante no tempo. 
Para facilitar a exposição da teoria supõe-se que todas as funções acima são analíticas. Em particular, há existência e unicidade de soluções pois o campo vetorial no membro direito de (2.1a) é de classe $\mathcal{C}^{1}$ [Sot79].

Simplificando a notação, a dependência de $t$ ou $x$ será omitida quando possível.

Serão denotadas por $y_{i}\left(0, x_{0}, t, u\right)$, para $i \in\{1, \ldots, m\}$, as saídas do sistema (2.1) no instante $t$, aplicando-se a entrada $u$, a partir de da condição inicial $x_{0}$ no instante $t_{0}=0$.

Definição 2.1 Para o sistema (2.1) uma saída $y_{i}$ é dita invariante sob uma entrada $u_{j}$ se para quaisquer $u=\left(u_{1}, \ldots, u_{j}, \ldots, u_{m}\right)$ e $\tilde{u}=\left(u_{1}, \ldots, \tilde{u}_{j}, \ldots, u_{m}\right)$.

$$
y_{i}\left(x_{0}, t, u\right)=y_{i}\left(x_{0}, t, \tilde{u}\right), \forall x_{0} \in \mathcal{A}, \forall t \in I
$$

Ou seja, a entrada $u_{j}$ não altera a saída $y_{i}$, independente da escolha de $u_{j}$.

Teorema 2.2 Considere o conjunto de funções abaixo:

$$
L_{g_{j}} L_{X_{1}} \ldots L_{X_{k}} h_{i}(x), \forall k \geq 0,\left(X_{1}, \ldots, X_{k}\right) \in\left\{f, g_{1}, \ldots, g_{m}\right\}, x \in \mathcal{A}
$$

Então, já que o sistema (2.1) é analítico, a saída $y_{i}$ é invariante sob uma entrada $u_{j}$ em $\mathcal{A}$ se e somente se (2.3) é identicamente nula para todo $x \in \mathcal{A}$.

Prova Vide [NS90]

Definição 2.3 O sistema (2.1) é chamado de desacoplado se, após possível reordenação das entradas, valem as seguintes propriedades para todo $i \in\{1, \ldots, m\}$ :

(i) A saida $y_{i}$ é invariante sob as entradas $u_{j}$, com $i \neq j$;

(ii) A saída $y_{i}$ é não invariante com respeito à entrada $u_{i}$.

Utilizando o Teorema 2.2 obtém-se uma condição necessária e suficiente para (i):

$$
\begin{gathered}
\forall j \neq i, L_{g_{j}} L_{X_{1}} \ldots L_{X_{k}} h_{i}(x) \equiv 0 \\
\forall k \geq 0,\left(X_{1}, \ldots, X_{k}\right) \in\left\{f, g_{1}, \ldots, g_{m}\right\}, x \in \mathcal{A}
\end{gathered}
$$

De (2.3), extrai-se também a influência de $u_{i}$ sobre $y_{i}$

$$
\begin{gathered}
L_{g_{i}} L_{X_{1}} \ldots L_{X_{k}} h_{i}(x) \\
\forall k \geq 0,\left(X_{1}, \ldots, X_{k}\right) \in\left\{f, g_{1}, \ldots, g_{m}\right\}, x \in \mathcal{A}
\end{gathered}
$$

Para (ii), considere-se o seguinte subconjunto de (2.5):

$$
L_{g_{i}} L_{f}^{k} h_{i}(x), \forall k \geq 0, x \in \mathcal{A}
$$

Proposição 2.4 Se vale o item (i) da Definição 2.3 e se (2.6) é identicamente nula para algum $i \in\{1, \ldots, m\}$, então:

$$
L_{g_{i}} L_{f}^{k} h_{i}(x) \equiv 0, \forall k \geq 0, x \in \mathcal{A}
$$

Então todas as funções em (2.5) também serão.

Prova Basta notar que a expressão (2.5) assume somente duas formas, para $r, s \geq 0$ e $x \in \mathcal{A}$.

Se $i \neq j: L_{g_{i}} L_{X_{1}} \ldots L_{X_{r}} \underbrace{L_{g_{j}}^{s} h_{i}(x)}_{\equiv 0} \equiv 0$, devido à (2.4).

Caso contrário: $L_{g_{i}} L_{X_{1}} \ldots L_{X_{r}} \underbrace{L_{g_{i}}^{s} h_{i}(x)}_{\equiv 0} \equiv 0$, devido à $(2.7)$ 
Corolário 2.5 Se um sistema (2.1) é desacoplado, então para todo $i \in\{1, \ldots, m\}$ :

$$
L_{g_{i}} L_{f}^{k} h_{i}(x) \neq 0, \text { para algum } k \geq 0 \text { e } x \in \mathcal{A}
$$

Prova Supor por absurdo que não, então seriam válidas as hipóteses do teorema anterior. Logo, todas as funções em (2.5) seriam nulas e o sistema seria desacoplado pois o item (i) da Definição não seria satisfeito.

Observação 2.6 (Derivações de Saída Sucessivas) Assumindo-se agora que o sistema (2.1) é desacoplado, a seguinte construção é admissivel para $i \in\{1, \ldots, m\}$ :

A partir de cada componente $y_{i}(x)=h_{i}(x)$ da saída, fixa-se $h_{i}^{(0)}(x) \doteq h_{i}(x)$.

Daí, derivando-se em relação a t, obtém-se:

$$
\begin{array}{rlr}
\dot{h}_{i}^{(0)} & =\frac{d}{d t}\left(h_{i}^{(0)}(x(t))\right) & \\
& =\frac{\partial h_{i}^{(0)}}{\partial x} \dot{x} & \\
& =\frac{\partial h_{i}^{(0)}}{\partial x}\left(f+\sum_{k=1}^{m} g_{k} u_{k}\right) & \\
& =\frac{\partial h_{i}^{(0)}}{\partial x} f+\sum_{k=1}^{m}\left(\frac{\partial h_{i}^{(0)}}{\partial x} g_{k}\right) u_{k} & \\
& =L_{f} h_{i}^{(0)}+\sum_{k=1}^{m}\left(L_{g_{k}} h_{i}^{(0)}\right) u_{k} & \\
& =L_{f} h_{i}+\sum_{k=1}^{m}\left(L_{g_{k}} h_{i}\right) u_{k} & \\
& =L_{f} h_{i}+u_{i} L_{g_{i}} h_{i} & \text { (por definição) } \\
& \doteq h_{i}^{(1)}(x, u) &
\end{array}
$$

De modo geral, enquanto $h_{i}^{(j)}$ for função somente de $x$, i.e., $h_{i}^{(j)}(x, u)=h_{i}^{(j)}(x)=L_{f}^{j} h_{i}(x)$, constrói-se $h_{i}^{(j+1)}$ derivando-se novamente:

$$
\begin{array}{rlr}
\dot{h}_{i}^{(j)} & =\frac{d}{d t}\left(h_{i}^{(j)}(x(t))\right) \\
& =\frac{\partial h_{i}^{(j)}}{\partial x} \dot{x} \\
& =\frac{\partial h_{i}^{(j)}}{\partial x}\left(f+\sum_{k=1}^{m} g_{k} u_{k}\right) & \\
& =\frac{\partial h_{i}^{(j)}}{\partial x} f+\sum_{k=1}^{m}\left(\frac{\partial h_{i}^{(j)}}{\partial x} g_{k}\right) u_{k} & \\
& =L_{f} h_{i}^{(j)}+\sum_{k=1}^{m}\left(L_{g_{k}} h_{i}^{(j)}\right) u_{k} & \\
& =L_{f} L_{f}^{j} h_{i}+\sum_{k=1}^{m}\left(L_{g_{k}} L_{f}^{j} h_{i}\right) u_{k} & \quad \text { (por construção) } \\
& =L_{f}^{j+1} h_{i}+u_{i} L_{g_{i}} L_{f}^{j} h_{i} & \left.\quad \text { (pois } L_{g_{k}} L_{f}^{j} h_{i}=0 \text { para } k \neq i \text { devido à }(2.4)\right) \\
& \doteq h_{i}^{(j+1)}(x, u)
\end{array}
$$

Finalmente, as sucessivas derivações geram uma expressão que depende explicitamente de u no estágio $\rho_{i}$. Isso ocorre necessariamente para algum $\rho_{i}$ finito, devido ao Corolário 2.5. Assim:

$$
\begin{aligned}
\dot{h}_{i}^{\left(\rho_{i}-1\right)} & =L_{f} h_{i}^{\left(\rho_{i}-1\right)}+u_{i} L_{g_{i}} h_{i}^{\left(\rho_{i}-1\right)} \\
& =L_{f}^{\rho_{i}} h_{i}+u_{i} L_{g_{i}} L_{f}^{\rho_{i}-1} h_{i} \\
& \doteq h_{i}^{\left(\rho_{i}\right)}(x, u)
\end{aligned}
$$


Onde $L_{g_{i}} L_{f}^{\rho_{i}-1} h_{i}(\bar{x}) \neq 0$ para algum $\bar{x} \in \mathcal{A}$

Utilizar-se-á também a notação alternativa $y_{i}^{(j)} \doteq h_{i}^{(j)}$ para $j>0$, enfatizando que essas expressões provém de sucessivas derivações das coordenadas de saída do sistema (2.1).

Definição 2.7 Nesse contexto, define-se o número característico $\rho_{i}$ como o menor inteiro não negativo tal que $y_{i}^{\left(\rho_{i}\right)}$ dependa explicitamente de $u$, ou seja, para $i \in\{1, \ldots, m\}$ :

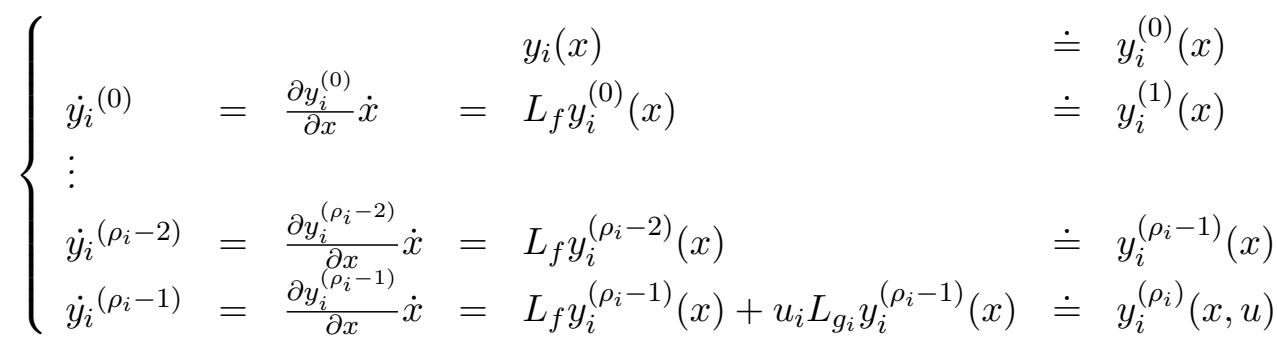

$$
\begin{aligned}
& \text { Observando que: }\left\{\begin{array}{ll}
L_{g_{i}} y_{i}^{(k)}(x) \equiv 0 & \forall x \in \mathcal{A}, \\
L_{g_{i}} y_{i}^{\left(\rho_{i}-1\right)}(\bar{x}) \neq 0 & \text { para algum } \bar{x} \in \mathcal{A}
\end{array} \quad \forall k \in\left\{0, \ldots, \rho_{i}-2\right\}\right.
\end{aligned}
$$

Note que aqui para todo $i \in\{1, \ldots, m\}, \rho_{i} \geq 1$ pois $h(x)$ não depende explicitamente de $u$ por hipótese.

Indica-se também o subconjunto:

$$
\mathcal{A}_{0} \doteq\left\{x \in \mathcal{A}: L_{g_{i}} y_{i}^{\left(\rho_{i}-1\right)}(x) \neq 0, \forall i \in\{1, \ldots, m\}\right\}
$$

No qual a entrada $u_{i}$ influencia instantaneamente a saída $y_{i}$

Definiremos também o grau relativo como a soma dos número característicos do sistema, i.e.:

$$
\rho \doteq \sum_{i=1}^{m} \rho_{i}
$$

Para exemplos simples desse processo de derivações de saída sucessivas, consultar Seções 4.2 e 4.3 .

Ao maior número característico entre $\left\{\rho_{1}, \ldots, \rho_{m}\right\}$, dá-se o nome de index do sistema (2.1), representando o "número de integrações entre" as entradas e saídas do sistema. Ele desempenha um papel importante no problema de desacoplamento, como será visto neste capítulo, além de fornecerem uma medida de dificuldade para a integração numérica desta classe de problemas.

Salienta-se que, diferentemente deste trabalho, algumas referências definem o número característico $\rho_{i}$ como o menor inteiro não negativo tal que $y_{i}^{\left(\rho_{i}+1\right)}$ dependa explicitamente de $u$ ao invés de $y_{i}^{\left(\rho_{i}\right)}$.

Definição 2.8 Um sistema (2.1) é dito fortemente desacoplado se vale o item (i) da Definição 2.3 e se existem números característicos $\rho_{1}, \ldots, \rho_{m}$ tais que se $\mathcal{A}_{0}=\mathcal{A}$.

Caso o item (i) não for satisfeito em $\mathcal{A}$ inteiro, mas se $x_{0} \in \mathcal{A}$ possuir uma vizinhança $\mathcal{V} \subset \mathcal{A}$ na qual o sistema é fortemente desacoplado, então diz-se que esta propriedade é local.

Em geral, (2.1) não é desacoplado, contudo pode-se tentar alterar a sua dinâmica adicionando uma realimentação de estado, como abaixo. 
Definição 2.9 Para o sistema (2.1) uma realimentação de estado estática tem o formato:

$$
u=\alpha(x)+\beta(x) v
$$

Onde $\alpha: \mathcal{A} \rightarrow \mathbb{R}^{m}, \beta: \mathcal{A} \rightarrow \mathbb{R}^{m \times m}$ e $v \in \mathbb{R}^{m}$.

Com $\operatorname{det} \beta(x) \neq 0$ para todo $x \in \mathcal{A}$. Aplicando-se a realimentação de estado (2.13) ao sistema (2.1), obtém-se o sistema modificado em malha fechada:

$$
\left\{\begin{array}{l}
\dot{x}=\tilde{f}(x)+\sum_{i=1}^{m} \tilde{g}_{i}(x) v_{i}(x) \\
y=h(x)
\end{array}\right.
$$

Onde:

$$
\begin{aligned}
\tilde{f}(x) & \doteq f(x)+\sum_{j=1}^{m} \tilde{g}_{j}(x) \alpha_{j}(x) \\
\tilde{g}_{i}(x) & \doteq \sum_{j=1}^{m} g_{j}(x) u_{i} \beta_{j i}(x) \\
v & \doteq \beta^{-1}(x) \alpha(x)+\beta^{-1}(x) u
\end{aligned}
$$

Denotando as operações matricialmente e substituindo (2.15) em (2.14a), obtém-se:

$$
\dot{x}=\tilde{f}+\tilde{g} v=(f+g \alpha)+(g \beta)\left(-\beta^{-1} \alpha+\beta^{-1} u\right)=f+g \alpha-g \alpha+g u=f+g u
$$

Logo, o sistema modificado é a expressão do sistema original com uma entrada reparametrizada por uma transformação, dada pela realimentação de estado estática.

Inclui-se abaixo o problema do desacoplamento que o primeiro método estudado neste trabalho busca resolver.

Problema 2.10 (Problema do Desacoplamento) Para o sistema (2.1), como obter uma realimentação de estado estática (2.13) tal que o sistema modificado (2.14) seja fortemente desacoplado?

Definição 2.11 Para um sistema (2.1) não necessariamente desacoplado é possivel aplicar-se derivações de saída sucessivas (Observação 2.6). Assim como no caso desacoplado $\dot{y}_{i}^{(j)}=L_{f} y_{i}^{(j)}(x)=$ $y_{i}^{(j+1)}$ para $j \in\left\{0, \ldots, \rho_{i}-2\right\}$. Já que agora não é garantido (2.4), tem-se:

$$
\begin{aligned}
\dot{y}_{i}^{\left(\rho_{i}-1\right)} & =L_{f} h_{i}^{\left(\rho_{i}-1\right)}+\sum_{k=1}^{m}\left(L_{g_{k}} h_{i}^{\left(\rho_{i}-1\right)}\right) u_{k} \\
& =L_{f} h_{i}^{\left(\rho_{i}-1\right)}+\left(L_{g_{1}} h_{i}^{\left(\rho_{i}-1\right)} \ldots L_{g_{m}} h_{i}^{\left(\rho_{i}-1\right)}\right)\left(\begin{array}{c}
u_{1} \\
\vdots \\
u_{m}
\end{array}\right) \\
& \doteq y_{i}^{\left(\rho_{i}\right)}
\end{aligned}
$$


Agrupando matricialmente esses resultados, indicar-se-á:

$$
y^{(\rho)}(x, u)=a(x)+b(x) u
$$

Onde:

$$
\begin{array}{r}
y(\rho) \doteq\left(\begin{array}{c}
y_{1}^{\left(\rho_{1}\right)} \\
\vdots \\
y_{m}^{\left(\rho_{m}\right)}
\end{array}\right), a(x) \doteq\left(\begin{array}{c}
L_{f} h_{1}^{\left(\rho_{1}-1\right)} \\
\vdots \\
L_{f} h_{m}^{\left(\rho_{m}-1\right)}
\end{array}\right), u \doteq\left(\begin{array}{c}
u_{1} \\
\vdots \\
u_{m}
\end{array}\right) \\
b(x) \doteq\left(\begin{array}{c}
L_{g} h_{1}^{\left(\rho_{1}-1\right)} \\
\vdots \\
L_{g} h_{m}^{\left(\rho_{m}-1\right)}
\end{array}\right) \doteq\left(\begin{array}{ccc}
L_{g_{1}} h_{1}^{\left(\rho_{i}-1\right)} & \ldots & L_{g_{m}} h_{1}^{\left(\rho_{i}-1\right)} \\
\vdots & & \vdots \\
L_{g_{1}} h_{m}^{\left(\rho_{i}-1\right)} & \ldots & L_{g_{m}} h_{m}^{\left(\rho_{i}-1\right)}
\end{array}\right)
\end{array}
$$

Identificando-se $\mathbb{R}^{m \times m}$ com o espaço das matrizes reais $m \times m, b(x) \in \mathbb{R}^{m \times m}$ recebe o nome de matriz de desacoplamento.

Observação 2.12 Observe que num sistema (2.1) fortemente desacoplado, para todo $x \in \mathcal{A}$ a matriz de desacoplamento $b(x)$ é:

- Diagonal, já que o item (i) da Definição 2.3 equivale a (2.4), então $L_{g_{i}} h_{j}^{\left(\rho_{i}-1\right)}=0$ para $i \neq j$.

- Tal que todos elementos de sua diagonal são não nulos em $\mathcal{A}_{0}$, pelo Corolário 2.5 e pela definição de $\mathcal{A}_{0}$ em (2.11), ressaltando que por ser um sistema fortemente desacoplado $\mathcal{A}=$ $\mathcal{A}_{0}$.

Lema 2.13 Uma realimentação de estado estática $u(x)=\alpha(x)+\beta(x) v$, não altera os número característicos de (2.1). Além disso, denotando a matriz de desacoplamento do sistema modificado por $\tilde{b}(x)$, e a da sistema original por $(x)$, vale que $\tilde{b}(x)=b(x) \beta(x)$.

Prova Vide [NS90]

Teorema 2.14 Considere-se o sistema (2.1), com números característicos $\rho_{1}, \ldots, \rho_{m}$. Então o Problema de Desacoplamento 2.10 pode ser resolvido por uma realimentação de estado estática (2.13) se e somente se:

$$
\operatorname{posto} b(x)=m, \forall x \in \mathcal{A}
$$

\section{Prova $(\Leftarrow)$}

Aplicando-se derivações de saída sucessivas obtém-se a expressão (2.18a) correspondente da forma $y^{(\rho)}(x, u)=a(x)+b(x) u$. Como assumimos que posto $b(x)=m, \forall x \in \mathcal{A}, b^{-1}(x)$ está bem definida neste domínio. Escolhe-se então a realimentação de estado estática:

$$
\begin{gathered}
u(x)=\alpha(x)+\beta(x) v \\
\operatorname{Com} \alpha(x)=-b^{-1}(x) a(x) \text { e } \beta(x)=b^{-1}(x)
\end{gathered}
$$

Assim:

$$
\begin{aligned}
y^{\rho}(x) & =a(x)+b(x) u \\
& =a(x)+b(x)(\alpha(x)+\beta(x) v) \\
& =a(x)+b(x)\left(-b^{-1}(x) a(x)+b^{-1}(x) v\right) \\
& =v
\end{aligned}
$$

Logo, o sistema em malha fechada (2.14) é desacoplado pois existem por hipótese $\rho_{1}, \ldots, \rho_{m}$ e cada uma das saídas $y_{i}$ é influenciada somente pela entrada $u_{i}$, para $i \in\{1, \ldots, m\}$.

$$
(\Rightarrow)
$$

A existência de uma solução para o Problema de Desacoplamento diz que é possível projetar uma 
realimentação de estado estática $u(x)=\alpha(x)+\beta(x) v$, com $\beta(x)$ não singular, tal que o sistema modificado (2.14) seja desacoplado. Ademais, pela Observação (2.12), a matriz de desacoplamento desse sistema modificado $\tilde{b}(x)$ possui posto máximo para todo $x \in \mathcal{A}$. Assim:

$$
\begin{array}{rlr}
0 & \neq \operatorname{det}(\tilde{b}(x)) & \\
& =\operatorname{det}(b(x) \beta(x)) & \\
& =\operatorname{det}(b(x)) \operatorname{det}(\beta(x)) &
\end{array}
$$

Portanto det $(b(x)) \neq 0$ e como $b(x)$ é uma matriz quadrada de tamanho $m$, conclui-se a tese.

Apresenta-se a seguir o teorema que, juntamente com as coordenadas complementares, possibilitará a obtenção da transformação de coordenadas sobre o espaço de estados necessária para a obtenção do método objeto deste trabalho. Esta prova é incluída para mostrar que a imposição de sistemas afins não será necessária posteriormente para o método.

Observação 2.15 Seja um sistema não linear explícito, dado por sua representação de estado:

$$
\left\{\begin{array}{l}
\dot{x}=f(x, u) \\
y=h(x, u)
\end{array}\right.
$$

Onde mantém-se a mesma notação e dimensões de (2.1), com a diferença que:

$f: \mathcal{A} \times \mathbb{R}^{m} \rightarrow \mathbb{R}^{n}$ é um campo vetorial analítico que descreve a dinâmica do sistema para o estado $x$ com entrada $u$.

$h: \mathcal{A} \times \mathbb{R}^{m} \rightarrow \mathbb{R}^{m}$ é uma função analítica que dá a saída do sistema.

Utilizaremos daqui por diante $\mathcal{S}$ para denotar $\mathcal{A} \times \mathbb{R}^{m}$, com $x \in \mathcal{A}$ e $u \in \mathbb{R}^{m}$ Por definição a derivada de Lie $L_{f} h_{i}(x)$ dá a derivada direcional da função $h_{i}$ na direção $f$ avaliada no ponto $x$, por isso não faria sentido à princípio tomar a direção $f$ dependente de uma variável externa u. Entretanto, abusando-se da notação, a expressão $y_{i}^{(j+1)}(x)=\frac{\partial}{\partial x}\left(y_{i}^{(j)}\right)(x)$ será indicada por $L_{f} y_{i}^{(j)}(x)$, para $i \in\{1, \ldots, m\}$ e $j \in\left\{0, \ldots, \rho_{i}-1\right\}$, no sentido de que por construção $y_{i}^{(j+1)}$ não depende explicitamente de u. Isto posto, pode-se de maneira similar à (2.10) aplicar derivações de saída sucessivas para (2.22), até obter-se uma expressão que dependa explicitamente de u, ou seja, para $i \in\{1, \ldots, m\}$ constrói-se:

$$
\begin{aligned}
& \begin{cases}h_{i}(x, u) & \doteq y_{i}^{(0)}(x) \\
\dot{y}_{i}{ }^{(0)}=\frac{\partial y_{i}^{(0)}}{\partial x} \dot{x}=L_{f} y_{i}^{(0)}(x) & \doteq y_{i}^{(1)}(x) \\
\vdots & \\
\dot{y}_{i}{ }^{\left(\rho_{i}-2\right)}=\frac{\partial y_{i}^{\left(\rho_{i}-2\right)}}{\partial x} \dot{x}=L_{f} y_{i}^{\left(\rho_{i}-2\right)}(x) & \doteq y_{i}^{\left(\rho_{i}-1\right)}(x) \\
\dot{y}_{i}{ }^{\left(\rho_{i}-1\right)}=\frac{\partial y_{i}^{\left(\rho_{i}-1\right)}}{\partial x} \dot{x}=L_{f} y_{i}^{\left(\rho_{i}-1\right)}(x, u) & \doteq y_{i}^{\left(\rho_{i}\right)}(x, u)\end{cases}
\end{aligned}
$$

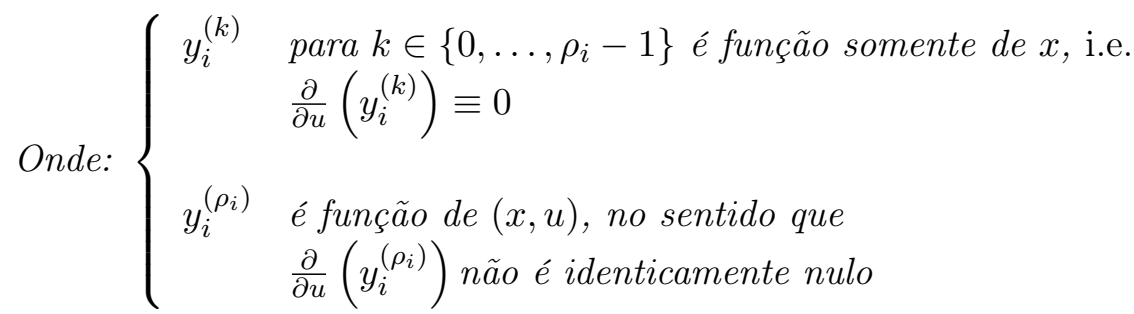

Mantendo as nomenclaturas de número característico e grau relativo (conforme (2.12)) como no caso afim. Observa-se somente que para (2.22) pode acontecer que $y_{i}^{(0)}=h_{i}$ depender explicitamente 
de $u$, para algum $i \in\{1, \ldots, m\}$, e neste caso $\rho_{i}=0$.

Outrossim, note que derivando-se (2.18a) em relação a u, obtém-se para a forma afim (2.1) uma outra expressão para a matriz de desacoplamento:

$$
\frac{\partial}{\partial u}\left(y^{(\rho)}\right)=\frac{\partial}{\partial u}(a(x)+b(x) u)=b(x)
$$

Ademais, para o sistema sem a restrição afim (2.22) denota-se a função que generaliza (2.18a), dada por $y^{(\rho)}: \mathcal{A} \times \mathbb{R}^{m} \rightarrow \mathbb{R}^{m}:$

$$
y^{(\rho)}(x, u) \doteq\left(\begin{array}{c}
L_{f} y_{1}^{\left(\rho_{1}-1\right)}(x, u) \\
\vdots \\
L_{f} y_{m}^{\left(\rho_{m}-1\right)}(x, u)
\end{array}\right)
$$

Por outro lado, salienta-se que a realimentação de estado estática definida aqui requer a hipótese de que o sistema seja afim. Este fato é utilizado fortemente na Definição 2.11 e na demonstração do Teorema 2.14 .

O argumento para a demonstração do próximo teorema é essencialmente o mesmo utilizado para o caso afim com posto máximo da matriz de desacoplamento.

Teorema 2.16 Para um sistema (2.22) com grau relativo $\rho$, tome-se a aplicação $Y^{(\rho-1)}: \mathcal{A} \rightarrow \mathbb{R}^{\rho}$ definida por:

$$
Y^{(\rho-1)}(x)=\left(y_{1}^{(0)}(x), \ldots, y_{1}^{\left(\rho_{1}-1\right)}(x), \ldots, y_{m}^{(0)}(x), \ldots, y_{m}^{\left(\rho_{m}-1\right)}(x)\right)
$$

Se o posto da matriz $\left.\frac{\partial}{\partial u}\left(y^{(\rho)}\right)\right|_{(x, u)}$ for máximo para $(x, u)$ no aberto $\mathcal{W} \doteq(\mathcal{U} \times \mathcal{V}) \subset \mathcal{S}$, então o posto da aplicação $\left.\frac{\partial}{\partial x}\left(Y^{(\rho-1)}\right)\right|_{(x)}$ para $x \in \mathcal{U}$ também será.

Prova Por absurdo, assuma que para algum $x \in \mathcal{U}$, o posto de $Y^{(\rho-1)}(x)$ não é máximo, ou seja, os vetores linha $\frac{\partial}{\partial x}\left(Y^{(\rho-1)}\right)$ devem ser linearmente dependentes. Para tanto, deve existir um vetor linha com $\rho$ constantes não todas nulas, denotado por $C=\left(c_{1}^{1}, \ldots, c_{1}^{\rho_{1}}, \ldots, c_{m}^{1}, \ldots, c_{m}^{\rho_{m}}\right)$ tal que:

$$
0_{(\rho \times 1)}=\left.C \frac{\partial}{\partial x}\left(Y^{(\rho-1)}\right)\right|_{(x)}
$$

A fim de não sobrecarregar a notação, tomam-se nesta prova todas as matrizes jacobianas avaliadas no ponto $x$. Toma-se também $u$ tal que $(x, u) \in \mathcal{W}$.

Multiplicando-se $\frac{\partial}{\partial x}\left(Y^{(\rho-1)}\right)$ por $f(x, u)$ à direita e substituindo os termos de (2.26), tem-se:

$$
\frac{\partial}{\partial x}\left(Y^{(\rho-1)}\right) f=\left(\begin{array}{c}
\frac{\partial}{\partial x}\left(y_{1}^{(0)}\right) \\
\vdots \\
\frac{\partial}{\partial x}\left(y_{1}^{\left(\rho_{1}-1\right)}\right) \\
\vdots \\
\frac{\partial}{\partial x}\left(y_{m}^{(0)}\right) \\
\vdots \\
\frac{\partial}{\partial x}\left(y_{m}^{\left(\rho_{m}-1\right)}\right)
\end{array}\right) f=\left(\begin{array}{c}
L_{f} y_{1}^{(0)} \\
\vdots \\
L_{f} y_{1}^{\left(\rho_{1}-1\right)} \\
\vdots \\
L_{f} y_{m}^{(0)} \\
\vdots \\
L_{f} y_{m}^{\left(\rho_{m}-1\right)}
\end{array}\right)=\left(\begin{array}{c}
y_{1}^{(1)} \\
\vdots \\
y_{1}^{\left(\rho_{1}\right)} \\
\vdots \\
y_{m}^{(1)} \\
\vdots \\
y_{m}^{\left(\rho_{m}\right)}
\end{array}\right)
$$

Assim, $C \frac{\partial}{\partial x}\left(Y^{(\rho-1)}\right)=0$ implica

$$
\sum_{i=1}^{m} \sum_{j=1}^{\rho_{i}} c_{i}^{j} y_{i}^{(j)}=0
$$


Derivando-se agora a expressão acima em relação à $u$ tem-se:

$$
\begin{aligned}
0 & =\frac{\partial}{\partial u}\left(\sum_{i=1}^{m} \sum_{j=1}^{\rho_{i}} c_{i}^{j} y_{i}^{(j)}\right) \\
& =\sum_{i=1}^{m} \sum_{j=1}^{\rho_{i}} c_{i}^{j} \frac{\partial}{\partial u}\left(y_{i}^{(j)}\right) \\
& =\sum_{i=1}^{m} c_{i}^{\rho_{i}} \frac{\partial}{\partial u}\left(y_{i}^{\left(\rho_{i}\right)}\right) \quad \text { pois } y_{i}^{(j)} \text { não depende de } u \text { para } j<\rho_{i} \\
& =\left(c_{1}^{j}, \ldots, c_{m}^{\rho_{m}}\right) \frac{\partial}{\partial u}\left(y^{(\rho)}\right)
\end{aligned}
$$

Visto que $\frac{\partial}{\partial u}\left(y^{(\rho)}\right)$ possui linhas linearmente independentes, conclui-se que as constantes $\left(c_{1}^{\rho_{1}}, \ldots, c_{m}^{\rho_{m}}\right)$ são nulas.

O problema agora resume-se a analisar:

$$
\sum_{i=1}^{m} \sum_{j=1}^{\rho_{i}-1} c_{i}^{j} y_{i}^{(j)}=0
$$

Para ver que as constantes $\left(c_{1}^{\rho_{1}-1}, \ldots, c_{m}^{\rho_{m}-1}\right)$ também são todas nulas, basta tomar a derivada da expressão acima por $x$ na direção $f$ e depois derivar em relação a $u$, pois:

$$
\begin{array}{rlrl}
0 & = & \frac{\partial}{\partial u}\left(\frac{\partial}{\partial x}\left(\sum_{i=1}^{m} \sum_{j=1}^{\rho_{i}-1} c_{i}^{j} y_{i}^{(j)}\right) f(x, u)\right) \\
& = & \frac{\partial}{\partial u}\left(\sum_{i=1}^{m} \sum_{j=1}^{\rho_{i}-1} c_{i}^{j} \frac{\partial}{\partial x}\left(y_{i}^{(j)}\right) f(x, u)\right) \\
& = & \frac{\partial}{\partial u}\left(\sum_{i=1}^{m} \sum_{j=1}^{\rho_{i}-1} c_{i}^{j} y_{i}^{(j+1)}\right) \\
& = & \sum_{i=1}^{m} c_{i}^{\rho_{i}-1} \frac{\partial}{\partial u}\left(y_{i}^{\left(\rho_{i}\right)}\right) \\
& = & & \left(c_{1}^{\rho_{1}-1}, \ldots, c_{m}^{\rho_{m}-1}\right) \\
\end{array}
$$

Novamente, como $\frac{\partial}{\partial u}\left(y^{(\rho)}\right)$ possui linhas linearmente independentes, conclui-se que as constantes $\left(c_{1}^{\rho_{1}-1}, \ldots, c_{m}^{\rho_{m}-1}\right)$ são nulas.

A aplicação repetida deste argumento mostra o resultado desejado.

\subsection{Existência de Coordenadas Complementares}

Pela seção anterior, obtém-se sob certas condições $\rho$ funções de $\mathbb{R}^{n}$ em $\mathbb{R}^{n}$ linearmente independentes. Vê-se abaixo que existem $n-\rho$ funções complementares, definindo uma transformação de coordenadas no espaço de estados.

Proposição 2.17 Seja $\varphi \doteq\left\{\varphi_{i}: \mathcal{A} \rightarrow \mathbb{R}^{n}, i \in\{1, \ldots, \rho\}\right\}$ um conjunto de funções suaves e independentes no sentido de que $\left.\mathrm{D} \varphi\right|_{(x)}$ possui posto máximo no aberto $\mathcal{A} \subset \mathbb{R}^{n}$. Então, para todo $x_{0} \in \mathcal{A}$ existe uma vizinhança $\mathcal{U} \subset \mathcal{A}$ de $x_{0}$ e outro conjunto de funções suaves $\hat{x}=$ $\left\{\hat{x}_{i}: \mathcal{U} \rightarrow \mathbb{R}, i \in\{1, \ldots, n-\rho\}\right\}$, tal que a aplicação $\psi: \mathcal{U} \rightarrow \mathbb{R}^{n}$ definida por:

$$
\psi(x)=(\hat{x}(x), \varphi(x))
$$

É um difeomorfismo entre $\mathcal{U}$ e $\psi(\mathcal{U})$ 
Prova Afirma-se que para $x_{0} \in \mathcal{U}$, qualquer função $\hat{x}$ de classe $\mathcal{C}^{1}$ tal que $\left.\frac{\partial \hat{x}}{\partial x}\right|_{x_{0}}$ possua vetores linha linearmente independentes dos vetores linha de $\left.\frac{\partial \varphi}{\partial x}\right|_{x_{0}}$ servirá. Assim:

$$
\left.\frac{\partial \psi}{\partial x}\right|_{x_{0}}=\left.\left(\begin{array}{c}
\frac{\partial \hat{x}}{\partial x} \\
\frac{\partial \varphi}{\partial x}
\end{array}\right)\right|_{x_{0}}
$$

É uma matriz de posto máximo em uma vizinhança de $x_{0}$. Então o Teorema da Função Inversa garante a existência de uma vizinhança $\mathcal{V}$ de $x_{0}$ tal que $\psi$ é um difeomorfismo entre $\mathcal{V}$ e $\psi(\mathcal{V})$.

Observe também que, por construção, $\psi(x)$ terá a menor classe de diferenciabilidade entre $\varphi$ e $\hat{x}$, funções estas de classe $\mathcal{C}^{k \geq 1}$

Para exemplificar o teorema, registra-se abaixo uma construção particular com $\hat{x}(x)$ linear que será utilizada no próximo capítulo.

Observação 2.18 Considere-se $x_{0} \in \mathcal{A}$ e $\hat{x}(x)=K x$. Assumindo-se que $\frac{\partial \varphi}{\partial x}$ possui posto máximo em $\mathcal{A}$, fixa-se $K_{(n-\rho) \times n}$ tal que a matriz $\left(\begin{array}{c}K \\ \frac{\partial \varphi}{\partial x}\end{array}\right)$ é não singular em $x_{0}$.

Assim, se $\psi(x)=(\hat{x}(x), \varphi(x))$, o determinante a matriz jacobiana $\left.\frac{\partial \psi}{\partial x}\right|_{x_{0}}$ é não nulo por construção. Já que a função determinante é contínua e $\frac{\partial \psi}{\partial x}$ é contínua por hipótese, existe uma vizinhança $\mathcal{V}$ de $x_{0}$ tal que $\left.\frac{\partial \psi}{\partial x}\right|_{x_{1}}$ ainda é não nulo para todo $x_{1} \in \mathcal{V}$.

Portanto, o Teorema da Função Inversa garante que existe uma outra vizinhança $\mathcal{U} \subset \mathcal{V}$ de $x_{0}$ tal que $\mathcal{U}$ e $\psi(\mathcal{U})$ são difeomórficos.

\subsection{Método por Realimentação de Estado Estática - MI}

Para introduzir as próximas, inclui-se aqui o método por realimentação de estado estática. Nesta seção consideram-se sistemas afins (2.1).

Reunindo os resultados do Teorema 2.16 e da Proposição 2.17, conclui-se que a condição posto $b(x)=m$ para todo $x$ pertencente a algum aberto $\mathcal{V} \subset \mathcal{A}$, é necessária e suficiente para que a aplicação $\psi: \mathcal{V} \rightarrow \mathbb{R}^{n}$ seja uma transformação de coordenadas diferenciável, que será denotada $z=\psi(x)$.

Derivando-se então $z(t)=\psi(x(t))$ e substituindo a expressão de $\dot{x}$ conforme (2.1a), tem-se:

$$
\begin{aligned}
\dot{z} & =\frac{d}{d t}(\psi(x)) \\
& =\frac{\partial \psi}{\partial x} \dot{x} \\
& =\frac{\partial \psi}{\partial x}(f(x)+g(x) u) \\
& =\frac{\partial \psi}{\partial x}(f(x))+\frac{\partial \psi}{\partial x}(g(x)) u \\
& =\frac{\partial \psi}{\partial x}\left(f\left(\psi^{-1}(z)\right)\right)+\frac{\partial \psi}{\partial x}\left(g\left(\psi^{-1}(z)\right)\right) u \\
& =\tilde{f}(z)+\tilde{g}(z) u
\end{aligned}
$$

Onde $\tilde{f}: \tilde{\mathcal{V}} \rightarrow \mathbb{R}^{n}$ e $\tilde{g}: \tilde{\mathcal{V}} \rightarrow \mathbb{R}^{m \times n}$ são as representações na coordenadas $z$ das funções $f$ e $g$, respectivamente.

Além disso, a soma $\sum_{i=1}^{m} g_{i}(x) u_{i}(x)$ foi indicada matricialmente por $g(x) u$.

Daí, as soluções das equações diferenciais (2.1a) e (2.33) conjugadas ([Sot79]) pela transformação $\psi$, no sentido de que se $x\left(t, x_{0}\right)$ é solução com condição inicial $x_{0}$ de $(2.1 \mathrm{a})$ então $\psi\left(x\left(t, x_{0}\right)\right)=$ 
$z\left(t, z_{0}\right)$ é solução $(2.33)$ com condição inicial, com $z_{0}=\psi\left(x_{0}\right)$ e vice-versa.

Como $\psi(x)=\left(\hat{x}(x), Y^{(\rho-1)}(x)\right)$ para alguma escolha de coordenadas complementares $\hat{x}$, reescrevese $z=\left(\hat{x}, Y^{(\rho-1)}\right)$. Levando à seguinte reformulação da equação $(2.1 \mathrm{a})$ nas coordenadas $\left(\hat{x}, Y^{(\rho-1)}\right)$ :

$$
\left\{\begin{array}{l}
\dot{\hat{x}}=\tilde{f}\left(\hat{x}, Y^{(\rho-1)}\right)+\tilde{g}\left(\hat{x}, Y^{(\rho-1)}\right) u \\
\left\{\begin{array}{l}
\dot{y}_{i}^{(0)}=y_{i}^{(1)} \\
\vdots \\
\dot{y}_{i}^{\left(\rho_{i}-2\right)}=y_{i}^{\left(\rho_{i}-1\right)} \\
\dot{y}_{i}^{\left(\rho_{i}-1\right)}=a_{i}+b_{i} u
\end{array}\right\}, \text { para } i \in\{1, . ., m\}
\end{array}\right.
$$

Onde foi utilizado o vínculo natural de diferenciações entre as coordenadas $y_{i}^{(j)}$, tal qual (2.10). Denotando-se também por $a_{i}$ e $b_{i}$ a i-ésima linha das funções definidas em (2.18).

Adicionando agora a realimentação de estado estática desacoplante $u(x)=\alpha(x)+\beta(x) v$, segundo (2.20), o Teorema 2.14 garante que o sistema (2.34) se torna:

$$
\left\{\begin{array}{l}
\dot{\hat{x}}=\theta\left(\hat{x}, Y^{(\rho-1)}, v\right) \\
\left\{\begin{array}{l}
\dot{y}_{i}^{(0)}=y_{i}^{(1)} \\
\vdots \\
\dot{y}_{i}^{\left(\rho_{i}-2\right)}=y_{i}^{\left(\rho_{i}-1\right)} \\
\dot{y}_{i}\left(^{\left(\rho_{i}-1\right)}=v_{i}\right.
\end{array}\right\}, \text { para } i \in\{1, . ., m\}
\end{array}\right.
$$

Onde $\theta\left(\hat{x}, Y^{(\rho-1)}, v\right) \doteq \tilde{f}\left(\hat{x}, Y^{(\rho-1)}\right)+\tilde{g}\left(\hat{x}, Y^{(\rho-1)}\right)(\alpha(x)+\beta(x) v), \operatorname{com} x=\psi^{-1}\left(\hat{x}, Y^{(\rho-1)}\right)$

Nestas novas coordenadas torna-se claro, tratando-se de sistemas quadrados, que ao escolher $v$ para controlar as coordenadas $Y^{(\rho-1)}$ não restam entradas para controlar diretamente $\hat{x}$. Por este motivo, e também devido à sua relação com os sistemas semi-implícitos como será visto na próxima seção, $\theta$ recebe o nome de dinâmica zero na literatura.

\subsection{Sistemas Explícitos versus Semi-Implícitos}

Considere o sistema (2.1) agora com o vínculo algébrico:

$$
\left\{\begin{array}{l}
\dot{x}=f(x)+g(x) u \\
y=h(x) \equiv 0
\end{array}\right.
$$

Ao contrário de antes, não mais podemos escolher arbitrariamente as entradas $u$ devido à restrição (2.36b). De um ponto de vista geométrico, pode-se dizer que as soluções desse sistema no espaço de estados versus entradas dado por $\mathcal{S} \doteq \mathcal{A} \times \mathbb{R}^{m}$ estão confinadas à superfície $\Gamma$, sendo implicitamente dependentes de $u$ pelas relação diferencial expressa por (2.36a).

Fixando a aplicação:

$$
\begin{array}{cccc}
Y^{(\rho)}: & \mathcal{S} & \rightarrow & \mathbb{R}^{n+m} \\
(x, u) & \mapsto & \left(\begin{array}{c}
Y^{(\rho-1)}(x) \\
y^{(\rho)}(x, u)
\end{array}\right)
\end{array}
$$


Onde $Y^{(\rho-1)}(x)$ é definida por $(2.25)$ e $y^{(\rho)}(x, u)$ é definida por $(2.26)$.

Indiquem-se os conjuntos:

$$
\Gamma_{\varepsilon} \doteq\left\{(x, u) \in \mathcal{S}:\left\|Y^{(\rho)}\right\| \leq \varepsilon, \varepsilon \geq 0\right\}
$$

Mantendo a notação $\Gamma=\Gamma_{0}$.

Além disso, o sistema (2.1) escrito em coordenadas $\left(\hat{x}, Y^{(\rho-1)}\right)$, conforme $(2.35)$ e com $v \equiv 0$, torna-se:

$$
\left\{\begin{aligned}
\dot{\hat{x}} & =\theta\left(\hat{x}, Y^{(\rho-1)}, 0\right) \\
\dot{Y}^{(\rho-1)} & =0
\end{aligned}\right.
$$

Neste contexto, para uma condição inicial $x_{0}$ tal que $h\left(x_{0}\right)=0$, as soluções dos sistemas $(2.39)$ e (2.36) são equivalentes, pois:

- A equação diferencial do sistema implícito(2.39a) é a mesma que a do sistema explícito (2.1a) e portanto igual à (2.36a) a menos difeomorfismo, como visto na seção anterior.

- A restrição de saída (2.36b) equivale (2.39b), pois ambas expressões para uma condição inicial em $\Gamma$ impõem que $y \equiv 0$, já que $Y^{(\rho)}(x, u)$ são derivadas de $y=h(x)$.

Com essa observação pode-se notar que as soluções $x(t)$ do sistema implícito (2.36) evoluem em $\Gamma$. Ademais, sobre uma destas soluções $\psi(x)=\left(\hat{x}(x), Y^{(\rho-1)}(x)\right)=(\hat{x}(x), 0)$, mostrando que $\Gamma$ é uma a subvariedade de dimensão $n-\rho$ em $\mathcal{S}$. Por essa razão, toda a informação necessária para o conhecimento das soluções está em $\hat{x}$, justificando o uso dos termos pseudo-estado e pseudo-entrada para $x$ e $u$

Pode-se, entretanto, não se dispor para fins computacionais de uma condição inicial justa na restrição $(2.36 \mathrm{~b})$, mas somente em $\Gamma_{\varepsilon}$, para algum $\varepsilon$ pequeno. Além disso, métodos para integração de (2.36) implementados em um computador de precisão finita acarretarão inevitavelmente erros numéricos, gerando o mesmo problema.

Para lidar com isso, considere-se a generalização de (2.39) tomando cada uma das entradas $v_{i}$, segundo um parâmetro de convergência $\gamma>0$ :

$$
v_{i}=-\sum_{j=0}^{\rho_{i}-1} c_{i}^{(j)} y_{i}^{(j)}, \forall i=\{1, . ., m\}
$$

Onde as constantes $c_{i}^{(j)}$ são os coeficientes do polinômio:

$$
\pi_{i}(s)=(s+\gamma)^{\rho_{i}}=s^{\rho_{i}}+\sum_{j=0}^{\rho_{i}-1} c_{i}^{(j)} s^{j}
$$

Aplicando então a entrada (2.40) ao sistema (2.35), obtém-se:

$$
\left\{\begin{array}{c}
\dot{\hat{x}}=\frac{\partial \hat{x}}{\partial x}(f(x)+g(x) u) \\
\left(\begin{array}{c}
\dot{y}_{i}{ }^{(0)} \\
\dot{y}_{i}{ }^{(0)} \\
\vdots \\
\dot{y}_{i}{ }^{\left(\rho_{i}-1\right)}
\end{array}\right)=\left(\begin{array}{ccccc}
0 & 1 & 0 & \ldots & 0 \\
0 & 0 & 1 & \ldots & 0 \\
\vdots & & & \ddots & \vdots \\
0 & 0 & 0 & \ldots & 1 \\
-c_{i}^{(0)} & & \ldots & & -c_{i}^{\left(\rho_{i}-1\right)}
\end{array}\right)\left(\begin{array}{c}
y_{i}^{(0)} \\
y_{i}^{(0)} \\
\vdots \\
y_{i}^{\left(\rho_{i}-1\right)}
\end{array}\right) \forall i \in\{1, . ., m\}
\end{array}\right.
$$


Haja vista que (2.41b) é linear e possui todos autovalores iguais a $-\gamma$, então $\lim _{t \rightarrow \infty} Y^{(\rho-1)}(t)=0$, [Sot79].

Daí, para uma condição inicial $x_{0} \operatorname{com}\left\|Y^{(\rho-1)}\left(x_{0}\right)\right\|<\varepsilon$ e $\varepsilon$ pequeno, a entrada (2.40) no sistema (2.41) atenua o erro em relação à $(2.36 \mathrm{~b})$, gerando um solução $x\left(t, x_{0}\right)$ do sistema explícito (2.1) que convirja para uma solução $x\left(t, x_{0}^{*}\right) \operatorname{com} Y^{(\rho-1)}\left(x_{0}^{*}\right)=0$, que é uma solução do sistema implícito (2.36).

Já que $\psi(x)=\left(\hat{x}, Y^{(\rho-1)}\right)$ implica $\psi(x) \dot{x}=\left(\dot{\hat{x}}, \dot{Y}^{(\rho-1)}\right)$ e $\psi(x)=\left(\begin{array}{c}\frac{\partial \hat{x}}{\partial x} \\ \frac{\partial Y^{(\rho-1)}}{\partial x}\end{array}\right)$ por construção, tomando a expressão do sistema (2.41) na coordenada $x$, resumindo os resultados até aqui, obtém-se a estrutura do Método por Realimentação de Estado Estática: (MI)

$$
\left\{\begin{aligned}
\left(\begin{array}{c}
\frac{\partial \hat{x}}{\partial x} \\
\frac{\partial Y^{(\rho-1)}}{\partial x}
\end{array}\right) \dot{x} & =\left\{\begin{array}{c}
\frac{\partial \hat{x}}{\partial x}(f(x)+g(x) u(x)) \\
y_{i}^{(1)}(x) \\
\vdots \\
y_{i}^{\left(\rho_{i}-1\right)}(x) \\
v_{i}(x)
\end{array}\right\} \text { para } i \in\{1, \ldots, m\} \\
(x(0), u(0)) & =\left(x_{0}, u_{0}\right), \text { condição inicial fixa em } \Gamma_{\varepsilon}, \text { com } \varepsilon \text { pequeno } \\
u(x) & =\alpha(x)+\beta(x) v(x), \text { realimentação conforme }(2.13) \\
v(x) & \text { conforme }(2.40), \text { para assegurar convergência }
\end{aligned}\right.
$$

Outrossim, o Teorema da Continuidade em Relação às Condições Iniciais para EDOs [Sot79] garante que num intervalo finito de tempo as soluções $x\left(t, x_{0}^{*}\right)$ e $x\left(t, x_{0}\right)$ estarão próximas, desde que $x_{0}$ e $x_{0}^{*}$ estejam próximas.

\section{Observação 2.19 (Sobre a Convergência do MI)}

Mesmo com a teoria assegurando a convergência do Método por Realimentação de Estado Estática, esta convergência é de certa forma indireta, pois ela é atingida através da estabilização uma cadeia de integradores. Tome por exemplo um sistema (2.41) com $m=1$ e $\rho=2$, para uma condição inicial $\left(y^{(0)}(0), y^{(1)}(0)\right)=\left(\varepsilon_{0}, \varepsilon_{1}\right)$, então $(2.41 b)$ será da forma:

$$
\left\{\begin{array}{l}
\dot{y}^{(0)}=y^{(1)} \\
y^{(1)}=-\gamma^{2} y^{(0)}-2 \gamma y^{(1)} \quad \text {, já que } \pi(s)=(s+\gamma)^{2}=s^{2}+2 s \gamma+\gamma^{2}
\end{array}\right.
$$

Cuja solução analítica é:

$$
\begin{aligned}
& y^{(0)}(t)=e^{-\gamma t}\left(\varepsilon_{0}+\left(\varepsilon_{0} \gamma+\varepsilon_{1}\right) t\right) \\
& y^{(1)}(t)=-e^{-\gamma t}\left(\left(\varepsilon_{0} \gamma+\varepsilon_{1}\right) \gamma t-\varepsilon_{1}\right)
\end{aligned}
$$

Como pode ser verificado por substituição.

Observa-se que de fato $\lim _{t \rightarrow \infty} Y^{(\rho-1)}(t)=0$, pela presença do termo exponencial. Entretanto, pode ocorrer que no período do transiente, entre o instante inicial e o momento que o erro seja suficientemente pequeno, o módulo de $Y^{(\rho-1)}(t)$ primeiro aumente para depois diminuir. Isto pode tornar a convergência mais lenta. 
Para fixar ideias, analisar-se-á o comportamento de $y^{(0)}(t)$. Derivando em relação $t$ :

$$
\begin{aligned}
\frac{d}{d t}\left(y^{(0)}(t)\right) & =-\gamma e^{-\gamma t}\left(\varepsilon_{0}+\varepsilon_{0} \gamma+\varepsilon_{1} t\right)+e^{-\gamma t}\left(\varepsilon_{0} \gamma+\varepsilon_{1}\right) \\
& =-e^{-\gamma t}\left(\varepsilon_{0} \gamma^{2} t+\gamma \varepsilon_{1} t-\varepsilon_{1}\right)
\end{aligned}
$$

Igualando a expressão acima a zero, tem-se:

$$
\begin{aligned}
0 & =\underbrace{-e^{-\gamma t}}_{\neq 0}\left(t\left(\varepsilon_{0} \gamma^{2}+\gamma \varepsilon_{1}\right)-\varepsilon_{1}\right) \\
\Rightarrow t & =\frac{\varepsilon_{1}}{\varepsilon_{0} \gamma^{2}+\gamma \varepsilon_{1}}
\end{aligned}
$$

Assumindo que $\varepsilon_{0}$ e $\varepsilon_{1}$ são positivos, então $y^{(0)}(t)$ possui um ponto de máximo em $\bar{t}=\frac{\varepsilon_{1}}{\gamma\left(\varepsilon_{0} \gamma+\varepsilon_{1}\right)}$.

Esse máximo será:

$$
\begin{aligned}
y^{(0)}(\bar{t}) & =e^{-\gamma \frac{\varepsilon_{1}}{\gamma\left(\varepsilon_{0} \gamma+\varepsilon_{1}\right)}}\left(\varepsilon_{0}+\left(\varepsilon_{0} \gamma+\varepsilon_{1}\right) \frac{\varepsilon_{1}}{\gamma\left(\varepsilon_{0} \gamma+\varepsilon_{1}\right)}\right) \\
& =e^{-\frac{\varepsilon_{1}}{\left(\varepsilon_{0} \gamma+\varepsilon_{1}\right)}}\left(\varepsilon_{0}+\frac{\varepsilon_{1}}{\gamma}\right) \\
& \doteq \bar{y}
\end{aligned}
$$

A figura abaixo ilustra o comportamento de $y^{(0)}(t)$ :

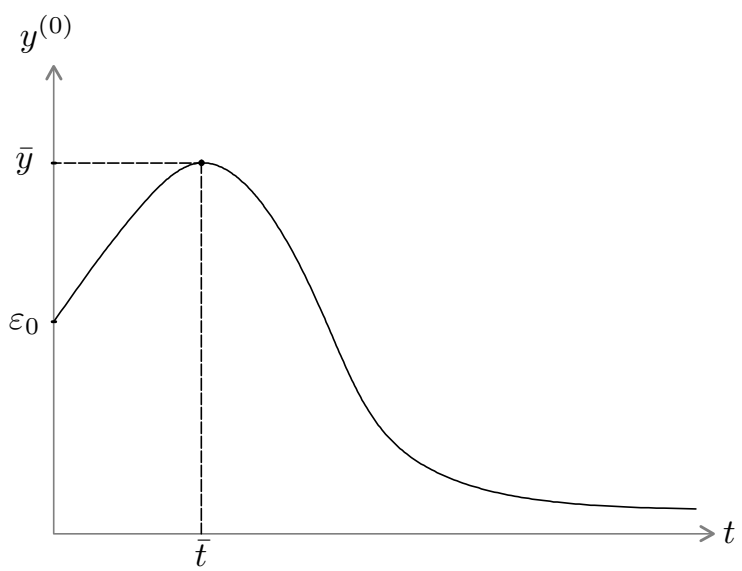

Figura 2.1: Exemplo de comportamento indesejado para o erro $y^{(0)}$ utilizando o MI.

Como $\left\|Y^{\rho}\right\|$ mede o erro referente à restrição (2.36b), deseja-se em particular que $y^{(0)}(\bar{t})$ seja minimo. É claro que se pode diminuir $y^{(0)}(\bar{t})$ aumentando o parâmetro $\gamma$ já que:

$$
\lim _{\gamma \rightarrow \infty} e^{-\frac{\varepsilon_{1}}{\left(\sigma_{0} \gamma+\varepsilon_{1}\right)}}\left(\varepsilon_{0}+\frac{\varepsilon_{1}}{\gamma}\right)=\varepsilon_{0}
$$

Entretanto, o aumento de $\gamma$ dificulta a integração numérica, gerando um fenômeno conhecido como stiffness, devido à imposição de uma dinâmica muito rápida para a equação diferencial em relação ao tamanho do passo de integração.

Alguns experimentos numéricos ilustrando este fenômeno para diferentes $\gamma$ 's, bem como a perda de acuidade da solução são encontrados em [Fre07] e em [SDS05].

Com o intuito atingir maior precisão na solução, estudar-se-á a partir da próxima seção a alternativa estruturada em [Sou06]. 


\subsection{Método por Injeção de Saída Generalizada - MII}

Nesta seção apresenta-se um método para a obtenção de um sistema explícito, a partir de um sistema semi-implícito:

$$
\left\{\begin{array}{l}
\dot{x}=f(x, u) \\
y=h(x, u) \equiv 0
\end{array}\right.
$$

Utilizando a mesma notação de (2.22), impondo a restrição de saída para (2.22b). Para este método, tentar-se-á construir uma campo vetorial $\tau$ conjuntamente sobre o espaço de estados cartesiano com o espaço de entradas:

$$
\frac{d}{d t}\left(\begin{array}{l}
x \\
u
\end{array}\right)=\tau(t, x, u)
$$

Alternativamente denotado por:

$$
\left\{\begin{array}{l}
\dot{x}=\tau_{x}(t, x, u) \\
\dot{u}=\tau_{u}(t, x, u)
\end{array}\right.
$$
(2.48)

De tal sorte que as soluções deste novo sistema convirjam para as soluções do sistema implícito

Inicialmente, vê-se um corolário das seções anteriores com a transformação de coordenadas a ser utilizada.

Corolário 2.20 Considere um aberto $\tilde{\mathcal{W}} \doteq(\tilde{\mathcal{U}} \times \tilde{\mathcal{V}}) \subset \mathcal{S}$, com $x \in \tilde{\mathcal{U}}$ e $u \in \tilde{\mathcal{V}}$, no qual $\frac{\partial y^{(\rho)}}{\partial x}$ possui posto máximo em $\tilde{\mathcal{W}}$. Tome-se a aplicação $\Psi: \tilde{\mathcal{W}} \rightarrow \mathbb{R}^{n+m}$ definida por:

$$
\Psi(x, u)=\left(\hat{x}(x), Y^{(\rho-1)}(x), y^{(\rho)}(x, u)\right)
$$

Para $Y^{(\rho)}(x, u)=\left(Y^{(\rho-1)}(x), y^{(\rho)}(x, u)\right)$, conforme (2.37), e uma escolha de coordenadas complementares $\hat{x}: \tilde{\mathcal{U}} \rightarrow \mathbb{R}^{n-\rho}$. Então para todo $(x, u) \in \tilde{\mathcal{W}}$ existe uma vizinhança $\mathcal{W} \doteq(\mathcal{U} \times \mathcal{V})$ de $(x, u), \operatorname{com} \mathcal{U} \subset \tilde{\mathcal{U}}$ e $\mathcal{V} \subset \tilde{\mathcal{V}}$, tais que $\mathcal{W} e \Psi(\mathcal{W})$ são difeomórficos.

Prova Denote-se $\left.\frac{\partial \Psi(x, u)}{\partial(\bar{x}, \bar{u})}\right|_{x, u}$ por $T(\bar{x}, \bar{u})$ pelo restante deste trabalho, então:

$$
T(x, u)=\left.\left(\begin{array}{cc}
\frac{\partial \hat{x}}{\partial x} & 0 \\
\frac{\partial Y^{(\rho-1)}}{\partial x} & 0 \\
\frac{\partial y^{(\rho)}}{\partial x} & \frac{\partial y^{(\rho)}}{\partial u}
\end{array}\right)\right|_{(x, u)}
$$

Note que $\frac{\partial \hat{x}}{\partial u}$ e $\frac{\partial Y^{(\rho-1)}}{\partial u}$ são ambos nulos pois $\hat{x}$ e $Y^{(\rho-1)}$ não dependem de $u$ por construção (vide (2.23) e Proposição 2.17). Ademais, pelo Teorema 2.16, tem-se que para todo $x \in \tilde{\mathcal{U}}$, $\left.\frac{\partial Y^{(\rho-1)}}{\partial x}\right|_{x}$ possui posto máximo.

Por consequência da Proposição 2.17, a submatriz quadrada $n \times n,\left(\begin{array}{c}\frac{\partial \hat{x}}{\partial x} \\ \frac{\partial Y^{(\rho-1)}}{\partial x}\end{array}\right)$ é não singular.

Como o outro bloco na diagonal de $T(x, u)$ é a matriz quadrada $m \times m$ dada por $\frac{\partial y^{(\rho)}}{\partial u}$, não singular por hipótese, tem-se que (2.52) é inversível, o que implica pelo Teorema da Função Inversa a existência de uma vizinhança $Z \subset \tilde{\mathcal{W}}$ de $(x, u)$ para a qual $Z$ e $\Psi(Z)$ são difeomórficos. Eventualmente diminuindo essa vizinhança, obtém-se $\mathcal{W} \doteq(\mathcal{U} \times \mathcal{V}) \subset Z$ no qual vale a tese.

Observação 2.21 Convém mencionar que para este método a hipótese de que todas funções sejam analíticas pode ser relaxada, pois será suficiente que a transformação $\Psi$ seja de classe $\mathcal{C}^{1}$, o que já será garantido se $f$ e $h$ forem de classe $\mathcal{C}^{k}$, com $k$ maior do o index do sistema. 
Diferentemente de (2.41), considere-se agora um sistema de equações diferenciais definido sobre o espaço $\left(\hat{x}, Y^{(\rho-1)}\right)$ conjuntamente com $y^{(\rho)}$, escolhendo um parâmetro de convergência $\gamma>0$.

$$
\left\{\begin{array}{l}
\dot{\hat{x}}=\theta\left(\hat{x}, Y^{(\rho)}\right) \doteq \frac{\partial \hat{x}}{\partial x}(f(x, u)) \\
\left\{\begin{array}{l}
\dot{y}_{i}^{(0)}=-\gamma y_{i}^{(0)} \\
\vdots \\
\dot{y}_{i}^{\left(\rho_{i}-1\right)} \\
\dot{y}_{i}^{\left(\rho_{i}\right)}=-\gamma y_{i}^{\left(\rho_{i}-1\right)}
\end{array}\right\} \text {, para } i \in\{1, . ., m\}
\end{array}\right.
$$

Onde $(x, u)=\Psi^{1}\left(\hat{x}, Y^{(\rho)}\right)$. Reescrevendo na forma vetorial, obtém-se a equação diferencial que é a base do método MII:

$$
\left\{\begin{aligned}
\dot{\hat{x}} & =\theta\left(\hat{x}, Y^{(\rho)}\right) \\
\dot{Y}^{(\rho)} & =-\gamma Y^{(\rho)}
\end{aligned}\right.
$$

É importante ressaltar que não foi aproveitado o vínculo diferencial $\dot{y}_{i}^{(j)}=y_{i}^{(j+1)}$, para $i \in$ $\{1, \ldots, m\}$ e $j \in\left\{0, \ldots, \rho_{i}-1\right\}$ inerente à construção dessas funções por derivações sucessivas de saída, segundo (2.23).

Ao invés disso, foi fixada a equação diferencial (2.54b), denominada de injeção generalizada de saída, com a vantagem de que a velocidade de $y_{i}^{(j)}$ é proporcional ao erro em relação àquela coordenada, pois a restrição algébrica $(2.48 \mathrm{~b})$ equivale $y_{i}^{(j)} \equiv 0$, pela seção anterior. Isto evita o fenômeno indesejado ilustrado na Observação (2.19) da norma de $y_{i}^{(j)}$ crescer durante o transiente.

Estes fatos são constatados pela expressão da solução analítica para $(2.54 \mathrm{~b}), y_{i}^{(j)}(t)=y_{i}^{(j)}(0) e^{\gamma t}$ para $i \in\{1, \ldots, m\}$ e $j \in\left\{0, \ldots, \rho_{i}-1\right\}$, em particular, as soluções de (2.54) evoluirão sempre em $\Gamma$ para uma equação condição inicial $\left(x_{0}, u_{0}\right) \in \Gamma$. Visto que $\lim _{t \rightarrow \infty} Y^{(\rho)}(t)=0$, as soluções do sistema (2.54) estarão próximas da solução do sistema implícito (2.48), como garantirão os teoremas na próxima seção.

Como para o MI, expressar-se-á (2.54) nas coordenadas originais, já que $\Psi(x, u)=\left(\hat{x}, Y^{(\rho)}\right)$ implica $T(x, u)\left(\begin{array}{c}\dot{x} \\ \dot{u}\end{array}\right)=\left(\begin{array}{c}\dot{\hat{x}} \\ \dot{Y}^{(\rho)}\end{array}\right)$. Tomando a representação do sistema (2.54) nas coordenadas $(x, u)$ obtém-se a estrutura objeto do presente trabalho, denominada Método por Injeção de Saída Generalizada, (MII):

$$
\left\{\begin{aligned}
&\left(\begin{array}{c}
\dot{x} \\
\dot{u}
\end{array}\right)= \tau(x, u) \\
&(x(0), u(0))=\left(x_{0}, u_{0}\right), \text { condição inicial fixa em } \Gamma_{\varepsilon}, \text { com } \varepsilon \text { pequeno } \\
& \text { Onde } \quad T(x, u) \tau(x, u)=\left(\begin{array}{c}
\frac{\partial \hat{x}}{\partial x}(f(x, u)) \\
-\gamma Y^{(\rho)}(x, u)
\end{array}\right) \text {, e } T(x, u) \text { não singular }
\end{aligned}\right.
$$

\subsection{Teoremas de Proximidade}

Apresentam-se agora teoremas que formalizam e resumem os conceitos de convergência motivados na seção anterior. 
Considere daqui por diante as nomenclaturas Sistema Implícito para (2.48) e MII para (2.55). Como ilustrado na seção anterior, o Sistema (2.54) equivale localmente ao MII no sentido de conjugação de equações diferenciais, através da transformação local de coordenadas $\Psi^{-1}$, e por isso esse sistema será denotado por MII'. Mais precisamente, tem-se a observação:

Observação 2.22 Considerem-se os seguintes sistemas:

$$
\begin{gathered}
\text { MII' } \\
\left\{\begin{array} { r l } 
{ \dot { \hat { x } } } & { = \theta ( \hat { x } , Y ^ { ( \rho ) } ) } \\
{ \dot { Y } ( \rho ) } & { = - \gamma Y ^ { ( \rho ) } }
\end{array} \quad \text { e } \left\{\left(\begin{array}{c}
\dot{x} \\
\dot{u}
\end{array}\right)=\tau(x, u)\right.\right.
\end{gathered}
$$

Seja $(x(t), u(t))$ uma solução de MII com condição inicial $\left(x_{0}, u_{0}\right)$, contida num aberto $\mathcal{U}$ no qual a aplicação $\Psi(x, u)=\left(\hat{x}(x), Y^{(\rho-1)}(x), y^{(\rho)}(x, u)\right.$, ) é um difeomorfismo sobre $\Psi(\mathcal{U})$.

Assim, ao definir-se $\left(\hat{x}(t), Y^{(\rho)}(t)\right) \doteq \Psi(x(t), u(t))$, obtém-se:

$$
\begin{aligned}
\left(\hat{x}, Y^{(\rho)}\right) & =\Psi(x, u) \\
\Leftrightarrow\left(\begin{array}{c}
\hat{\hat{x}} \\
\dot{Y}^{(\rho)}
\end{array}\right) & =\frac{\partial \Psi}{\partial(x, u)}\left(\begin{array}{c}
\dot{x} \\
\dot{u}
\end{array}\right) \quad \text { derivando em } t \\
\Leftrightarrow\left(\begin{array}{c}
\dot{\hat{x}} \\
\dot{Y}^{(\rho)}
\end{array}\right) & =\left(\begin{array}{c}
\theta\left(\hat{x}, Y^{(\rho)}\right) \\
-\gamma Y^{(\rho)}
\end{array}\right) \text { pela definição de } \tau
\end{aligned}
$$

Portanto, $\left.\left(\hat{x}(t), Y^{(\rho)}(t)\right)\right)$ é solução de $M I I^{\prime}$ com condição inicial $\left(\hat{x}_{0}, Y_{0}^{(\rho)}\right) \doteq \Psi\left(x_{0}, u_{0}\right)$, para todo $t$ desde que $(x(t), u(t)) \in \mathcal{U}$. Para a recíproca, o desenvolvimento é análogo.

Além disso, a hipótese de que $\frac{\partial y^{(\rho)}}{\partial u}$ é não nula no domínio da função $\Psi$ é suficiente (vide Corolário 2.20) para que haja uma vizinhança sobre todo ponto no domínio da $\Psi$ tal que a aplicação $\Psi$ conjugue as soluções de MII em MII'.

Teorema 2.23 Seja $\bar{x}(t) \in \mathcal{C}^{\infty}$ uma solução do Sistema Implícito definida para $t \in I$, com entrada $\bar{u}(t) e \frac{\partial y^{(\rho)}}{\partial(x, u)}$ não singular sobre um aberto $\mathcal{V} \subset \mathcal{S}$ contendo $(\bar{x}(t), \bar{u}(t)), \forall t \in I$, então $\zeta(t) \doteq$ $(\bar{x}(t), \bar{u}(t))$ é uma solução para o MII com condição inicial $\zeta(0) \in \Gamma$. Reciprocamente, se $\zeta(t)=$ $(\bar{x}(t), \bar{u}(t))$ é uma solução para o MII com $t \in I, \zeta(0) \in \Gamma$ e $\frac{\partial y^{(\rho)}}{\partial(x, u)}$ não singular sobre um aberto $\mathcal{V} \subset \mathcal{S}$ contendo $\zeta(t), \forall t \in I$, então $x(t) \doteq \bar{x}(t)$ é uma solução para o Sistema Implícito com entrada $\bar{u}(t)$.

Prova Prova da primeira parte do teorema:

Seja $\overline{\bar{x}}(t) \in \mathcal{C}^{\infty}$ uma solução do Sistema Implícito definida para $t \in I$, com entrada $\bar{u}(t)$ e condição inicial $\left(x_{0}, u_{0}\right) \in \mathcal{V}$. Tome-se um instante $t_{1} \in I$ qualquer e o ponto $\left(x_{1}, u_{1}\right) \doteq\left(\bar{x}\left(t_{1}\right), \bar{u}\left(t_{1}\right)\right)$.

Como $\frac{\partial y^{(\rho)}}{\partial(x, u)}$ é não singular sobre o aberto $\mathcal{V}$ contendo essa solução, o Teorema 2.16 afirma que $\frac{\partial Y^{(\rho-1)}}{\partial x}$ tem posto máximo e pela Proposição $2.17, \psi(x)=\left(\hat{x}(x), Y^{(\rho-1)}(x)\right)$ é um difeomorfismo local entre uma vizinhança $\mathcal{U} \subset \operatorname{proj}_{x} \mathcal{V}$ de $x_{1}$ e $\psi(\mathcal{U})$. Considere-se $I_{1} \subset I$ tal que para todo $t$ em $I_{1}, x(t)$ pertença à $\mathcal{U}$.

Fixa-se agora a notação $\left(\hat{x}(t), Y^{(\rho-1)}(t)\right)$ para $\psi(\bar{x}(t))$. Daí, por diferenciação em relação a $t$ :

$$
\left(\begin{array}{c}
\dot{\hat{x}} \\
\dot{Y}^{(\rho-1)}
\end{array}\right)=\frac{\partial \psi}{\partial x} \dot{\bar{x}}=\left(\begin{array}{c}
\frac{\partial \hat{x}}{\partial x} \\
\frac{\partial Y^{(\rho-1)}}{\partial x}
\end{array}\right) f(\bar{x}, \bar{u})
$$

Por outro lado, $\dot{Y}^{(\rho-1)}$ foi construído de acordo com (2.23) por derivações sucessivas das saídas 
do Sistema Implícito, a saber:

$$
\left\{\begin{array}{l}
\dot{y}_{i}{ }^{(0)}=y_{i}^{(1)} \\
\vdots \\
\dot{y}_{i}^{\left(\rho_{i}-2\right)}=y_{i}^{\left(\rho_{i}-1\right)} \\
\dot{y}_{i}^{\left(\rho_{i}-1\right)}=y_{i}^{\left(\rho_{i}\right)}
\end{array}\right\} \text {, para } i \in\{1, . ., m\}
$$

Ademais, a restrição $h(\bar{x}(t), \bar{u}(t)) \equiv 0$ equivale à $Y^{(\rho)}(\bar{x}(t), \bar{u}(t))=0$ para todo $t \in I_{1}$, pois ambas expressões impõem que a saída do Sistema Implícito y seja nula, já que as funções de $Y^{(\rho)}$ são da forma $y_{i}^{(j)}=L_{f}^{j} h_{i}$, para $i \in\{1, \ldots, m\}$ e $j \in\left\{0, \ldots, \rho_{i}\right\}$.

Combinando estes resultados e denotando $\frac{\partial \hat{x}}{\partial x} f(x, u)$ por $\theta\left(\hat{x}, Y^{(\rho)}\right)$, conforme (2.53), vê-se que $\left(\hat{x}(t), Y^{(\rho-1)}(t)\right)$ satisfaz:

$$
\left\{\begin{aligned}
\dot{\hat{x}} & =\theta\left(\hat{x}, Y^{(\rho)}\right) \\
Y^{(\rho)}(t) & \equiv 0
\end{aligned}\right.
$$

Portanto $\left(\hat{x}(t), Y^{(\rho-1)}(t)\right)$ é solução de MII' para todo $t \in I_{1}$ com $t_{1} \in I_{1}$, visto que a solução do sistema $\left\{\dot{Y}^{(\rho)}=-\gamma Y^{(\rho)} ; Y^{(\rho)}\left(t_{1}\right)=0\right\}$ é nula.

Pela Observação 2.22, existe uma vizinhança $\mathcal{W} \subset \mathcal{V}$ de $\left(\bar{x}\left(t_{1}\right), \bar{u}\left(t_{1}\right)\right)$ no qual a aplicação $\Psi$ conjuga soluções entre MII e MII'. Tome-se $\mathcal{W}$ pequeno o suficiente para que $\operatorname{proj}_{x} \mathcal{W}$ esteja contida em $\mathcal{U}$. Indique-se então $\zeta(t) \doteq \Psi^{-1}\left(\hat{x}(t), Y^{(\rho-1)}(t), y^{(\rho)}(t)\right)$, função esta bem definida para $t \in I_{1}$ tal que $(\bar{x}(t), \bar{u}(t)) \in \mathcal{W}$.

Notando que $\Psi(x, u)=\left(\psi(x), y^{(\rho)}(x, u)\right)=\left(\hat{x}(x), Y^{(\rho-1)}(x), y^{(\rho)}(x, u)\right)=\left(\hat{x}(x), Y^{(\rho)}(x, u)\right)$, tem-se que:

$$
\zeta(t)=\Psi^{-1}\left(\left(\hat{x}(t), Y^{(\rho)}(t)\right)\right)=\Psi^{-1} \circ \Psi \circ(\bar{x}, \bar{u})(t)=\left(\begin{array}{c}
\bar{x}(t) \\
\bar{u}(t)
\end{array}\right)
$$

Assim, $\zeta(t)$ é uma solução do MII em um subintervalo de $I$ contendo $t_{1}$. Como esse $t_{1}$ foi escolhido arbitrariamente em $I, \zeta(t)=(\bar{x}(t), \bar{u}(t)) \forall t \in I$ pois vale a existência e unicidade das soluções para MII, já que $\tau(x, u)$ é um campo suave.

Prova da segunda parte do teorema:

Seja $\zeta(t)=(\bar{x}(t), \bar{u}(t))$ uma solução para o MII com $t \in I$ e condição inicial $\zeta(0) \in \Gamma$.

Seja $t_{1} \in I$ qualquer e o ponto $\left(x_{1}, u_{1}\right) \doteq\left(\bar{x}\left(t_{1}\right), \bar{u}\left(t_{1}\right)\right)$. Já que $\frac{\partial y^{(\rho)}}{\partial(x, u)}$ é não singular no aberto $\mathcal{V}$ contendo os pontos desta solução, pela Observação 2.22 tem-se que existe uma vizinhança $\mathcal{W} \subset \mathcal{V}$ de $\left(x_{1}, u_{1}\right)$ tal que $\left(\hat{x}(t), Y^{(\rho-1)}, y^{(\rho)}(t)\right) \doteq \Psi(\zeta(t))$ é uma solução de MII' conjugada à $\zeta(t)$ no intervalo $I_{1} \doteq\{t \in I:(\bar{x}(t), \bar{u}(t)) \in \mathcal{W}\}$.

Nessas condições, $\psi(x)=\left(\hat{x}(x), Y^{(\rho-1)}(x)\right)$ é um difeomorfismo local entre uma vizinhança $\mathcal{U} \subset \operatorname{proj}_{x} \mathcal{V}$ de $x_{1}$ e $\psi(\mathcal{U})$. Tome-se $\mathcal{U}$ suficiente pequeno para que $\mathcal{U}$ esteja contido na $\operatorname{proj}_{x} \mathcal{W}$. Além disso, denote-se $x(t) \doteq \psi^{-1}\left(\hat{x}(t), Y^{(\rho-1)}(t)\right)$, função esta bem definida para $t \in I_{1}$ tal que $\bar{x}(t) \in \mathcal{U}$, resultando que:

$$
(\dot{x})=\frac{\partial \psi^{-1}}{\partial x}\left(\begin{array}{c}
\dot{\hat{x}} \\
\dot{Y}^{(\rho-1)}
\end{array}\right)=\frac{\partial \psi^{-1}}{\partial x}\left(\begin{array}{c}
\frac{\partial \hat{x}}{\partial x} f(\bar{x}, \bar{u}) \\
-\gamma Y^{(\rho-1)}(\bar{x}, \bar{u})
\end{array}\right)=\frac{\partial \psi^{-1}}{\partial x} \frac{\partial \psi}{\partial x} f(\bar{x}, \bar{u})=f(\bar{x}, \bar{u})
$$

Utilizando na penúltima igualdade que $-\gamma Y^{(\rho-1)}(\bar{x}, \bar{u}) \equiv 0 \equiv \frac{\partial Y^{(\rho-1)}}{\partial x} f(\bar{x}, \bar{u})$, já que $\frac{\partial Y^{(\rho-1)}}{\partial x} f(\bar{x}, \bar{u})$ são derivadas da saída $y=h(\bar{x}, \bar{u}) \equiv 0$

Portanto $x(t)$ é solução do Sistema Implícito com entrada $\bar{u}(t)$ num subintervalo de $I$ contendo $t_{1}$. 
Já que $\operatorname{proj}_{\left(\hat{x}, Y^{(\rho-1)}\right)}(\Psi(x, u))=\operatorname{proj}_{\left(\hat{x}, Y^{(\rho-1)}\right)}\left(\hat{x}(x), Y^{(\rho-1)}(x), y^{(\rho)}(x, u)\right)=\left(\hat{x}(x), Y^{(\rho-1)}(x)\right)=$ $\psi(x)$, tem-se:

$$
\begin{aligned}
x(t) & =\psi^{-1}\left(\hat{x}, Y^{(\rho-1)}\right) \\
& =\psi^{-1}\left(\operatorname{proj}_{\left(\hat{x}, Y^{(\rho-1)}\right)}(\Psi(\bar{x}(t), \bar{u}(t)))\right) \\
& =\psi^{-1} \circ \psi \circ \bar{x}(t) \\
& =\bar{x}(t)
\end{aligned}
$$

Assim $x(t)=\bar{x}(t)$ para todo $t \in I$ porque $t_{1}$ foi escolhido arbitrariamente em $I$, lembrando que vale a existência e unicidade das soluções para Sistema Implícito, já que $f(x, u)$ é um campo suave. $\square$

Na prova anterior, as únicas propriedades exigidas para $\hat{x}: \mathcal{V} \rightarrow \mathbb{R}^{\rho}$ são que ela seja de classe $\mathcal{C}^{2}$, para existência e unicidade das soluções de MII'; e que os vetores linha de $\frac{\partial \hat{x}}{\partial x}$ sejam linearmente independentes de $\frac{\partial Y^{(\rho-1)}}{\partial x}$, para que $\left(\hat{x}, Y^{(\rho-1)}\right)$ sejam um difeomorfismo local.

Como a escolha de coordenadas complementares $\hat{x}$ não é única, a princípio ela poderia gerar campos vetoriais $\tau(x, u)$ distintos, pois $\tau$ foi construído utilizando-se $\hat{x}$. O corolário abaixo esclarece que isto não ocorre para pontos em $\Gamma$.

Corolário 2.24 (Independência de $\tau$ em relação às coordenadas complementares) O campo $\tau(x, u)$ definido no MII tem as seguintes propriedades que não dependem da escolha de $\hat{x}(x)$ :

1. $\dot{Y}^{(\rho)}=-\gamma Y^{(\rho)}$

2. Para um ponto $(\bar{x}, \bar{u}) \in \Gamma, \tau(\bar{x}, \bar{u})$ não depende da escolha de $\hat{x}(x)$

3. Para um ponto $(\bar{x}, \bar{u}) \in \Gamma, \dot{x}=\tau_{x}(\bar{x}, \bar{u})=f(\bar{x}, \bar{u})$, onde $\tau_{x}$ corresponde às primeiras $n$ coordenadas do campo $\tau$.

Prova Seja $\hat{x}(x)$ uma escolha qualquer de coordenadas complementares conforme a Proposição 2.17. Então vale:

1. Por construção, já que a imposição da dinâmica $-\gamma Y^{(\rho)}$ foi fixada sem depender de $\hat{x}$ no MII'.

2. Pois se $(x(t), u(t))$ é uma solução para o Sistema Implícito e $\zeta(t)$ é uma solução para o MII, ambos com condição inicial $(\bar{x}, \bar{u}) \in \Gamma$, então, pelo Teorema 2.23, $\operatorname{proj}_{x} \zeta(t)=x(t)$, independente da escolha de $\hat{x}(x)$.

3. Pois, no mesmo contexto do item anterior, diferenciando $\operatorname{proj}_{x} \zeta(t)=x(t)$ em relação a $t$ em $t=0$, tem-se $\left.\frac{d}{d t}(x(t))\right|_{t=0}=\tau_{x}(\bar{x}, \bar{u})=f(\bar{x}, \bar{u})$.

Por outro lado, a escolha de $\hat{x}(x)$ influencia na estabilidade numérica do método e proporciona diferentes interpretações geométricas para pontos $(\bar{x}, \bar{u}) \in \Gamma_{\varepsilon}$, como será retomado no próximo capítulo.

Teorema 2.25 (Convergência de $Y^{(\rho)}$ ) Seja $\zeta(t)=(\bar{x}(t), \bar{u}(t)$ ) uma solução para o MII com condição inicial $\zeta_{0}$. Se $\zeta(t)$ está bem definida para todo $t$ no intervalo $I \doteq\left[0, t_{f}\right]$, então

$$
\left\|Y^{(\rho)}(t)\right\| \leq e^{-\gamma t}\left\|Y^{(\rho)}(0)\right\| \text {, para todo } t \in I .
$$

Prova Observe primeiro que $Y^{(\rho)}(t)$ é igual à $\operatorname{proj}_{Y(\rho)} \Psi(\zeta(t))$ por construção. Já que $\dot{Y}^{(\rho)}=$ $-\gamma Y^{(\rho)}$ em MII', extrai-se a solução analítica desta equação diferencial:

$$
y_{i}^{(j)}(t)=y_{i}^{(j)}(0) e^{-\gamma t} \text { para } i \in\{1, \ldots, m\} \text { e } j \in\left\{0, \ldots, \rho_{i}-1\right\}
$$

Matricialmente, portanto: $\left\|Y^{(\rho)}(t)\right\| \leq e^{-\gamma t}\left\|Y^{(\rho)}(0)\right\|$, para todo $t \in I$. 
Inclui-se agora uma observação lembrando que se $\frac{\partial y^{(\rho)}}{\partial u}$ é não singular, garantem-se a existência dos difeomorfismos locais $\Psi$ e $\psi$ utilizados na hipótese do teorema seguinte.

Observação 2.26 Se a matriz jacobiana $\left.\frac{\partial y^{(\rho)}}{\partial u}\right|_{\left(x_{0}, u_{0}\right)}$ é não singular para todo $\left(x_{0}, u_{0}\right) \in \tilde{\mathcal{W}}=$ $(\tilde{\mathcal{U}} \times \tilde{\mathcal{V}}) \subset \mathcal{S}$, então:

- Pela Proposição 2.17, existem uma vizinhança $\mathcal{U}_{1} \subset \tilde{\mathcal{U}}$ de $x_{0}$ e coordenadas complementares suaves $\hat{x}: \mathcal{U}_{1} \rightarrow$ tais que a aplicação $\psi: \mathcal{U}_{1} \rightarrow \mathbb{R}^{n}$, dada por $\psi(x)=\left(\hat{x}(x), Y^{(\rho-1)}(x)\right)$ é um difeomorfismo entre $\mathcal{U}_{1}$ e $\psi\left(\mathcal{U}_{1}\right)$.

- Pelo Corolário 2.20 existe uma vizinhança $\mathcal{W}_{2}=\left(\mathcal{U}_{2} \times \mathcal{V}\right) \subset \tilde{\mathcal{W}}$ de $\left(x_{0}, u_{0}\right)$ tal que a aplica$c ̧ \tilde{a} o \Psi: \mathcal{W}_{2} \rightarrow \mathbb{R}^{m+n}$, dada por $\Psi(x, u)=\left(\hat{x}(x), Y^{(\rho-1)}(x), y^{(\rho)}(x, u)\right)$, com as coordenadas complementares $\hat{x}$ do item anterior, é um difeomorfismo entre $\mathcal{W}_{2}$ e $\Psi\left(\mathcal{W}_{2}\right)$.

- Considerando os abertos $\mathcal{U} \doteq \mathcal{U}_{1} \cap \mathcal{U}_{2}$ e $\mathcal{W} \doteq(\mathcal{U} \times \mathcal{V})$, vale simultaneamente que $\mathcal{U}$ e $\psi(\mathcal{U})$ são difeomórficos, bem como $\mathcal{W}$ e $\Psi(\mathcal{W})$.

Teorema 2.27 Seja $\mathcal{W}=(\mathcal{U} \times \mathcal{V}) \subset \mathcal{S}$ um aberto, conexo e limitado, onde $\mathcal{U} \subset \mathcal{A} \subset \mathbb{R}^{n}$ e $\mathcal{V} \subset \mathbb{R}^{m}$, tal que:

- $A$ aplicação $\Psi: \mathcal{W} \rightarrow \mathbb{R}^{m+n}$, dada por $\Psi(x, u)=\left(\hat{x}(x), Y^{(\rho-1)}(x), y^{(\rho)}(x, u)\right)$ é um difeomorfismo entre $\mathcal{W}$ e $\Psi(\mathcal{W})$.

- A aplicação $\psi: \mathcal{U} \rightarrow \mathbb{R}^{n}$, dada por $\psi(x)=\left(\hat{x}(x), Y^{(\rho-1)}(x)\right)$ é um difeomorfismo entre $\mathcal{U} e$ $\psi(\mathcal{U})$.

Então se $(x(t), u(t))$ é uma solução do MII com condição inicial $(x(0), u(0))=\left(x_{0}, u_{0}\right)$, definida num intervalo de tempo $I_{1}=\left[0, t_{1}\right]$ tal que $(x(t), u(t)) \in \mathcal{W}, \forall t \in I_{1}$; e se $\bar{x}(t)$ é uma solução para o Sistema Implícito com entrada $\bar{u}(t)$ e condição inicial $\left(x_{0}^{*}, u_{0}^{*}\right) \doteq \Psi^{-1}\left(\hat{x}\left(x_{0}\right), 0\right)$, definida num intervalo de tempo $I_{2}=\left[0, t_{2}\right]$ tal que $x(t) \in \mathcal{U}, \forall t \in I_{2}$, então vale a desigualdade:

$$
\|(x(t), u(t))-(\bar{x}(t), \bar{u}(t))\| \leq \kappa_{1}\left\|Y_{0}^{(\rho)}\right\| e^{-\gamma t}+\kappa_{2}\left\|Y_{0}^{(\rho)}\right\|, \forall t \in I
$$

Onde $\kappa_{1}, \kappa_{2}$ são constantes não negativas e $I=\left[0, t_{f}\right] \doteq I_{1} \cap I_{2}$.

Prova Em primeiro lugar, note-se que para uma condição inicial em $\Gamma$ as soluções do Sistema Implícito são equivalentes às soluções do MII, no sentido expresso pelo Teorema 2.23. Logo, podemse tomar as respectivas expressões em coordenadas $\left(\hat{x}, Y^{(\rho)}\right)$ para o MII de solução $(\bar{x}(t), \bar{u}(t))$, com condição inicial $\left(x_{0}^{*}, u_{0}^{*}\right) \in \Gamma$, e para MII de solução $(x(t), u(t))$, com condição inicial $\left(x_{0}, u_{0}\right)$ :

$$
\left\{\begin{array} { r l } 
{ \dot { \hat { x } } } & { = \theta ( \hat { x } , 0 ) } \\
{ \hat { x } ( 0 ) } & { = \hat { x } _ { 0 } }
\end{array} \quad \text { e } \left\{\begin{array}{rl}
\dot{\hat{x}} & =\theta\left(\hat{x}, Y_{0}^{(\rho)} e^{-\gamma t}\right) \\
\hat{x}(0) & =\hat{x}_{0}
\end{array}\right.\right.
$$

Onde no primeiro sistema foi utilizado que $Y^{(\rho)}(x, u) \equiv 0$ para o Sistema Implícito e, no segundo sistema, foi substituída a expressão analítica da solução de $\dot{Y}^{(\rho)}=-\gamma Y^{(\rho)}$, conforme (2.25); visto as soluções de MII' e MII são conjugadas mesmo para condições iniciais em $\Gamma_{\varepsilon} \operatorname{com} \varepsilon$ pequeno, conforme Observação 2.22 e indicando $Y^{(\rho)}\left(x_{0}, u_{0}\right)$ por $Y_{0}^{(\rho)}$.

Vê-se que os dois sistemas são da forma:

$$
\left\{\begin{aligned}
\dot{\hat{x}} & =\tilde{\theta}(t, \hat{x}, \mu) \doteq \theta\left(\hat{x}, \mu e^{-\gamma t}\right) \\
\hat{x}(0) & =\hat{x}_{0}
\end{aligned}\right.
$$

Onde $\mu$ é um parâmetro fixo em $\mathbb{R}^{\rho+m}$, observando que $\tilde{\theta}$ é suave em relação $\mu$, com $\mu=0$ para o primeiro caso e $\mu=Y_{0}^{(\rho)}$ para o segundo. Portanto, a solução desse sistema na condição 
inicial $\hat{x}_{0}$ está bem definida, pode ser denotada por $\eta(t, \mu)$ no instante $t$ sob o parâmetro $\mu$ e, mais importante ainda, $\eta$ é diferenciável em relação à $\mu$ (vide [Pon62]). Com essa notação, pode-se voltar para coordenada $(x, u)$, obtendo $(\bar{x}(t), \bar{u}(t))=\tilde{\eta}(t, 0)$ e $(x(t), u(t))=\tilde{\eta}\left(t, Y_{0}^{(\rho)} e^{-\gamma t}\right)$. Onde:

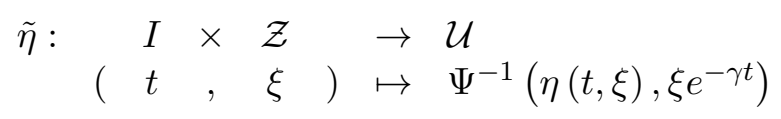

$\operatorname{Com} \mathcal{Z} \doteq \operatorname{proj}_{Y(\rho)}(\Psi(\mathcal{W}))$ e $I=I_{1} \cap I_{2}$ é um intervalo no qual $\left(\eta(t, \xi), \xi e^{-\gamma t}\right)$ pertence à $\Psi(\mathcal{W})$ para os dois sistemas. Salientando que a função $\operatorname{proj}_{Y(\rho)}: \mathbb{R}^{n-\rho} \times \mathbb{R}^{\rho+m} \rightarrow \mathbb{R}^{\rho+m}$ é dada por $\operatorname{proj}_{Y^{(\rho)}}\left(\hat{x}, Y^{(\rho)}\right)=Y^{(\rho)}$. Portanto, $\tilde{\eta}$ é diferenciável por ser composta de funções diferenciáveis. Então, vale que:

$$
\begin{aligned}
\|(\bar{x}(t), \bar{u}(t))-(x(t), u(t))\| & =\left\|\tilde{\eta}(t, 0)-\tilde{\eta}\left(t, Y_{0}^{(\rho)}\right)\right\| \\
& =\left\|\Psi^{-1}(\eta(t, 0), 0)-\Psi^{-1}\left(\eta\left(t, Y_{0}^{(\rho)}\right), Y_{0}^{(\rho)} e^{-\gamma t}\right)\right\| \\
& \leq\left\|\Psi^{-1}(\eta(t, 0), 0)-\Psi^{-1}\left(\eta(t, 0), Y_{0}^{(\rho)} e^{-\gamma t}\right)\right\| \\
& +\left\|\Psi^{-1}\left(\eta\left(t, Y_{0}^{(\rho)}\right), Y_{0}^{(\rho)} e^{-\gamma t}\right)-\Psi^{-1}\left(\eta(t, 0), Y_{0}^{(\rho)} e^{-\gamma t}\right)\right\| \\
& \leq \kappa_{1}\left\|Y_{0}^{(\rho)}\right\| e^{-\gamma t}+\kappa_{2}\left\|Y_{0}^{(\rho)}\right\|, \forall t \in I
\end{aligned}
$$

Utilizando duas vezes na última passagem a Desigualdade do Valor Intermediário.

Com a notação $[a, b]$ indicando o segmento de reta em $\mathbb{R}^{n}$ ligando os pontos $a, b$, ou seja $[a, b] \doteq\left\{(a+(b-a) t) \in \mathbb{R}^{n}: 0 \leq t \leq 1\right\}$, as constantes $\kappa_{1}$ e $\kappa_{2}$ podem ser tomadas como:

$$
\begin{aligned}
& \kappa_{1}=\max \left\{\left\|\left.\frac{\partial \Psi^{-1}}{\partial Y^{(\rho)}}\right|_{\left(\hat{x}, Y^{(\rho)}\right)}\right\|:\left(\hat{x}, Y^{(\rho)}\right) \in\left[(\eta(t, 0), 0),\left(\eta(t, 0), Y_{0}^{(\rho)} e^{-\gamma t}\right)\right]\right\} \\
& c_{1}=\max \left\{\left\|\left.\frac{\partial \Psi^{-1}}{\partial \hat{x}}\right|_{\left(\hat{x}, Y^{(\rho)}\right)}\right\|:\left(\hat{x}, Y^{(\rho)}\right) \in\left[\left(\eta\left(t, Y_{0}^{(\rho)}\right), Y_{0}^{(\rho)} e^{-\gamma t}\right),\left(\eta(t, 0), Y_{0}^{(\rho)} e^{-\gamma t}\right)\right]\right\} \\
& c_{2}=\max \left\{\left\|\left.\frac{\partial \eta}{\partial \mu}\right|_{(t, \mu)}\right\|:(t, \mu) \in\left[(t, 0),\left(t, Y_{0}^{(\rho)}\right)\right]\right\} \\
& \kappa_{2}=c_{1} c_{2}
\end{aligned}
$$

Além disso, através do Teorema 2.27, demonstrou-se um resultado a princípio inesperado. Sob certas condições, para todo instante da simulação $t_{1}$, existe um intervalo compacto $\left[t_{1}, t_{2}\right]$ no qual a distância entre a solução do MII e uma solução do Sistema Implícito decai exponencialmente até uma constante proporcional à $Y^{(\rho)}\left(x\left(t_{1}\right), u\left(t_{1}\right)\right)$, i.e.. proporcional ao erro da solução $(x(t), u(t))$ gerada pelo MII em relação à variedade $\Gamma$, no instante $t_{1}$. 


\section{Capítulo 3}

\section{Sistema Explícito Numérico}

No capítulo anterior viu-se como obter o Sistema Explícito MII (2.55) da forma:

$$
\left(\begin{array}{c}
\dot{x} \\
\dot{u}
\end{array}\right)=\tau(x, u)
$$

a partir do sistema semi-implícito quadrado autônomo (2.48), a saber:

$$
\left\{\begin{array}{l}
\dot{x}=f(x, u) \\
y=h(x, u) \equiv 0
\end{array}\right.
$$

Onde $f: \mathcal{S} \rightarrow \mathbb{R}^{n}$ e $h: \mathcal{S} \rightarrow \mathbb{R}^{m}$ são funções suaves, definidas no aberto $\mathcal{S}=\mathcal{A} \times \mathbb{R}^{m}$ no qual admite-se que $\frac{\partial y^{(\rho)}}{\partial u}$ possui posto máximo em $\mathcal{S}$ de modo que, pelo Corolário 2.20, a aplicação $\Psi: \mathcal{S} \rightarrow \mathbb{R}^{m+n}$ seja um difeomorfismo local.

Apresentam-se aqui algoritmos em pseudo-código para construir esse campo vetorial $\tau(x, u)$, utilizando cálculos simbólicos e numéricos, possibilitando posteriormente o uso de métodos tradicionais para resolução numérica de EDOs. Os códigos fonte dos algoritmos escritos para Matlab ${ }^{\circledR}$ utilizando o pacote de operações simbólicas constam no Apêndice.

Lembrando, conforme Seção 2.2, que a estrutura do Sistema Explícito MII não é única por requerer uma escolha arbitrária de coordenadas complementares $\hat{x}: \mathcal{A} \rightarrow \mathbb{R}^{n-\rho}$, discutem-se então duas alternativas de escolhas numéricas.

Ambas abordagens apresentadas utilizam o complemento ortogonal extraído de uma decomposição QR, sendo que na primeira o cálculo é feito em cada um dos instantes da solução, enquanto na segunda ele é refeito apenas quando a matriz $T(x, u)=\frac{\partial \Psi}{\partial(x, u)}$ perde condicionamento.

Ao final do capítulo registra-se uma generalização simples do MII permitindo tratar sistemas variantes no tempo e a construção das funções necessárias para a simulação.

\subsection{Coordenadas por Derivação de Saída}

Utilizando cálculos simbólicos, o primeiro trabalho a ser feito é aplicar o processo (2.23), derivando em relação a $t$ sucessivamente as componentes $y_{i}^{(0)} \doteq h_{i}$, para $i \in\{1, \ldots, m\}$, da função de saída (2.48b). Definindo iterativamente funções $y_{i}^{(k+1)}$ enquanto a expressão anterior for função somente da variável $x$ e parando no primeiro estágio $\rho_{i}<\infty$ tal que $y_{i}^{\left(\rho_{i}\right)}$ depende explicitamente de $u$. Construindo-se daí $Y^{(\rho)}(x, u)=\left(Y^{(\rho-1)}(x), y^{(\rho)}(x, u)\right)$, com $Y^{(\rho-1)}: \mathcal{A} \rightarrow \mathbb{R}^{n-\rho}$ e $y^{(\rho)}: \mathcal{S} \rightarrow \mathbb{R}^{m}$.

\section{Observação 3.1 (Notação alternativa para as funções do MII)}

Neste capítulo fixam-se os seguintes símbolos: rho $=\rho, Y=Y^{(\rho-1)}, Y x=\frac{\partial Y^{(\rho-1)}}{\partial x}, y p=y^{(\rho)}$, ypx $=\frac{\partial y^{(\rho)}}{\partial x}$ e ypu $=\frac{\partial y^{(\rho)}}{\partial u}$. Essa notação será utilizada nos algoritmos e também na exposição da teoria quando isso não prejudicar o entendimento. 
A notação $a \leftarrow b$ indica que é atribuído à variável $a$ o conteúdo da variável $b$. Múltiplos comandos por linha são separados por um ponto e vírgula.

Já que as matrizes $\frac{\partial Y^{(\rho-1)}}{\partial x}, \frac{\partial y^{(\rho)}}{\partial x}$ e $\frac{\partial y^{(\rho)}}{\partial u}$ calculadas para a obtenção de $y^{(\rho)}$ e $Y^{(\rho-1)}$ são utilizadas também na matriz $T(x, u)$, elas também são devolvidas pelo algoritmo.

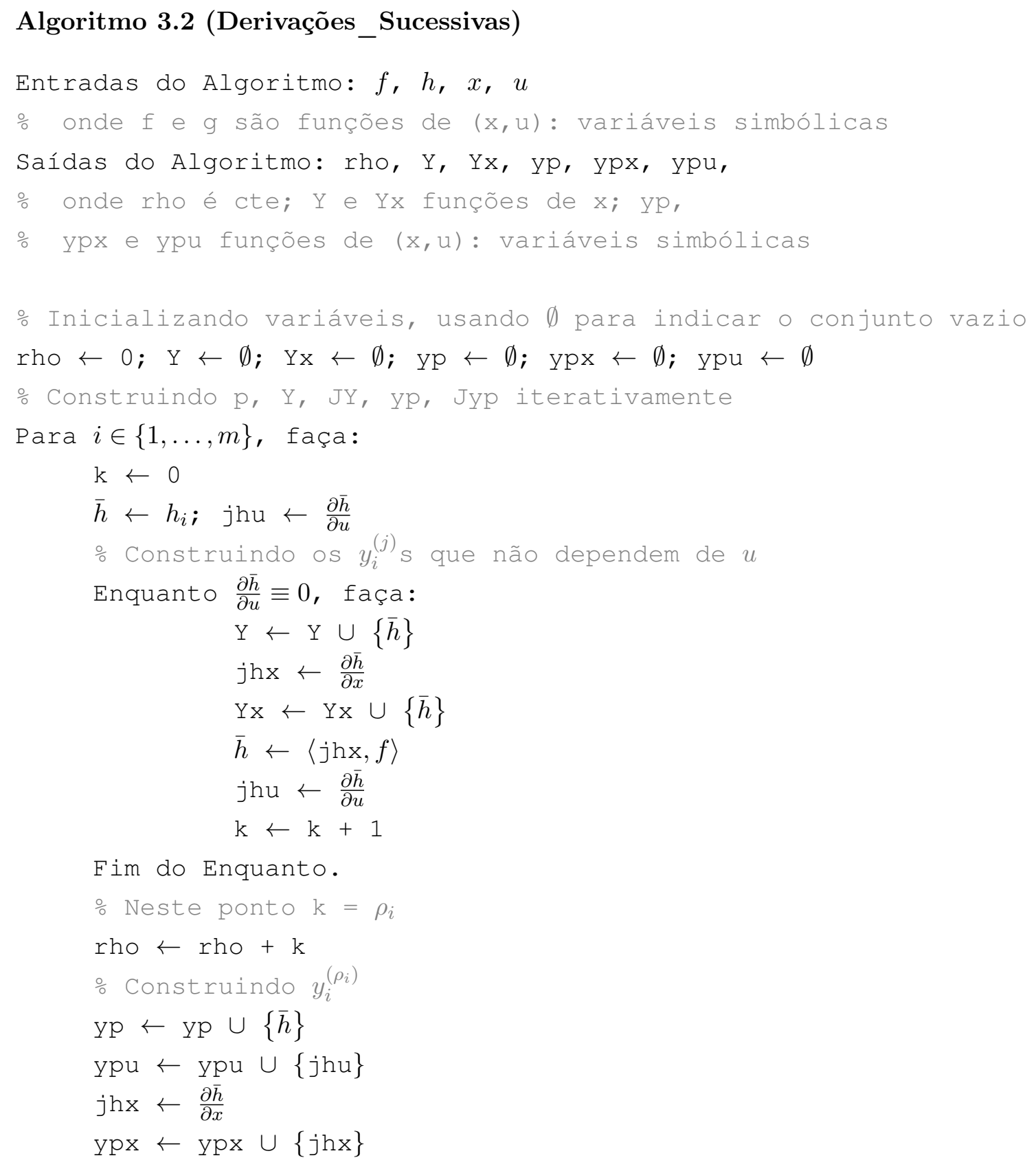

Fim do Para.

Uma observação importante é que a condição $\frac{\partial \bar{h}}{\partial u} \equiv 0$ depende de simplificações simbólicas, podendo chegar a uma conclusão enganosa se o pacote de cálculos simbólicos não estiver programado para identificar alguma equivalência necessária. Um exemplo simples seria se $\frac{\partial \bar{h}}{\partial u}=$ $\left(\operatorname{sen}^{2}(x)+\cos ^{2}(x)\right)-1$, onde é claro que $\frac{\partial \bar{h}}{\partial u} \equiv 0$. Contudo, se a expressão entre parêntesis fosse mais complicada e o pacote simbólico não a reconhecesse como sendo igual a 1, o algoritmo daria resultados errados. 


\subsection{Complemento Ortogonal}

Como visto na demonstração do Teorema 2.17, uma condição necessária e suficiente para que uma função suave $\hat{x}: \mathcal{U} \subset \mathcal{A} \rightarrow \mathbb{R}^{n-\rho}$ seja tal que $\psi(x)=\left(\hat{x}(x), Y^{(\rho-1)}(x)\right)$ é um difeomorfismo local é que os vetores linha de $\frac{\partial \hat{x}}{\partial x}$ sejam linearmente independentes dos vetores linhas de $\frac{\partial Y^{(\rho-1)}}{\partial x}$ para todo $x \in \mathcal{U}$.

Por outro lado, a estrutura do MII conforme (2.55) pode ser reescrita como:

$$
\left(\begin{array}{c}
\dot{x} \\
\dot{u}
\end{array}\right)=\tau(x, u)=\left(\begin{array}{cc}
\frac{\partial \hat{x}}{\partial x} & 0 \\
\frac{\partial Y^{(\rho-1)}}{\partial x} & 0 \\
\frac{\partial y^{(\rho)}}{\partial x} & \frac{\partial y^{(\rho)}}{\partial u}
\end{array}\right)^{-1}\left(\begin{array}{c}
\frac{\partial \hat{x}}{\partial x}(f(x, u)) \\
-\gamma Y^{(\rho)}(x, u)
\end{array}\right)
$$

De onde conclui-se que, ainda que o MII exija uma escolha suave local de coordenadas complementares $\hat{x}$, o valor do campo $\tau$ avaliado em cada ponto $(x, u) \in \mathcal{S}$ requer somente o conhecimento da matriz jacobiana $\frac{\partial \hat{x}}{\partial x}$ avaliada em $x$. Esse tema será retomado na próxima seção.

Veja-se agora que uma escolha pontual de vetores linearmente independentes de $\frac{\partial Y^{(\rho-1)}}{\partial x}$ pode ser feita numericamente através do complemento ortogonal.

Definição 3.3 (Complemento Ortogonal) Se $V$ é um espaço vetorial em $\mathbb{R}^{n}$, dá-se o nome de complemento ortogonal de $V$ ao conjunto indicado por $V^{\perp}=\left\{x \in \mathbb{R}^{n}:\langle x, v\rangle=0, \forall v \in V\right\}$.

Lema 3.4 Seja $V$ um espaço vetorial em $\mathbb{R}^{n}$, então $\mathbb{R}^{n}=V \oplus V^{\perp}$.

Observação 3.5 (Complemento Ortogonal via Decomposição QR) Com essa notação, um processo para se obter uma base ortogonal para $V^{\perp}$ é a decomposição QR [Str80]. Dada uma matriz de posto máximo $A_{n \times s}=\left[a_{1}, \ldots, a_{s}\right]$, com $n \geq s$, cujas colunas formam uma base de $V$, obtêm-se pela decomposição $Q R$ uma matriz ortogonal $Q_{n \times n}$ e uma matriz triangular superior $\tilde{R}_{n \times s}$, tais que $Q \tilde{R}=A$.

Como $A$ é retangular, pode-se ainda subdividir as matrizes $Q=\left[\begin{array}{ll}Q_{1} & Q_{2}\end{array}\right]$ e $\tilde{R}=\left[\begin{array}{c}R_{1} \\ 0\end{array}\right]$ de tal forma que:

$$
A=Q \tilde{R}=\left[\begin{array}{ll}
Q_{1} & Q_{2}
\end{array}\right]\left[\begin{array}{c}
R_{1} \\
0
\end{array}\right]=Q_{1} R_{1}
$$

Com $Q_{1}$ de dimensão $n \times s, Q_{2}$ de dimensão $n \times(n-s), R_{1}$ de dimensão $s \times s$ triangular superior de posto máximo.

Assim, similarmente ao processo de Gramm-Schmidt, a decomposição QR define nas colunas $\left\{u_{1}, \ldots, u_{s}\right\}$ de $Q_{1}$ uma base ortogonal para $V$. Além disso, como $Q$ é ortogonal, vale que:

$$
I_{n \times n}=Q^{\top} Q=\left[\begin{array}{c}
Q_{1}^{\top} \\
Q_{2}^{\top}
\end{array}\right]\left[\begin{array}{ll}
Q_{1} & Q_{2}
\end{array}\right]=\left[\begin{array}{cc}
Q_{1}^{\top} Q_{1} & Q_{1}^{\top} Q_{2} \\
Q_{2}^{\top} Q_{1} & Q_{2}^{\top} Q_{2}
\end{array}\right] \Rightarrow Q_{1}^{\top} Q_{2}=0_{s \times(n-s)}
$$

Logo $A^{\top} Q_{2}=\left(Q_{1} R_{1}\right)^{\top} Q_{2}=R_{1}^{\top} Q_{1}^{\top} Q_{2}=0 ;$ e os vetores coluna $\left\{v_{1}, \ldots, v_{n-s}\right\}$ de $Q_{2}$ são ortogonais entre si e pertencem ao complemento ortogonal de $V$. Mais ainda, como o lema anterior garante que $\mathbb{R}^{n}=V \oplus V^{\perp}$, então as colunas de $Q_{2}$ formam uma base ortonormal para $V^{\perp}$.

Outra observação pertinente, é que a decomposição $Q R$ fornece uma decomposição como em (3.3), contudo o complemento ortogonal $Q_{2}$ não é único, no sentido de que se uma matriz $Q_{2}^{\prime}$ é formada tomando-se reordenações e trocas de sinais dos vetores linhas de $Q_{2}, Q_{2}^{\prime}$ também será um complemento ortogonal.

Voltando para o contexto do MII, denote-se a matriz jacobiana $\frac{\partial Y^{(\rho-1)}}{\partial x}$ avaliada num ponto $x \in \mathcal{A}$ por $Y_{x}$, cuja dimensão é $\rho \times n$. Para vetores linha de $Y_{x}$ linearmente independentes, deseja-se 
calcular uma base para o complemento ortogonal do subespaço gerado pelas linhas de $Y_{x}$, assim:

$$
\begin{aligned}
& Y_{x}^{\top}=Q \tilde{R} \\
\Leftrightarrow & Y_{x}=\tilde{R}^{\top} Q^{\top}=\left[\begin{array}{ll}
r & 0
\end{array}\right]\left[\begin{array}{c}
q \\
R
\end{array}\right]=r q
\end{aligned}
$$

Onde, relacionando com (3.3), fixam-se as notações $r \doteq R_{1}^{\top}$ é uma matriz quadrada não singular $\rho \times \rho$ cuja transposta é triangular superior; $q \doteq Q_{1}^{\top}$ é uma matriz $\rho \times n$ e $R \doteq Q_{2}^{\top}$ é uma matriz $(n-\rho) \times n$.

Abusando-se da nomenclatura, será dito que $\mathrm{R}$ é o complemento ortogonal de $Y_{x}$ para indicar que as colunas de $R^{\top}$ são uma base para o complemento ortogonal do subespaço gerado pelas colunas de $Y_{x}^{\top}$.

Da Observação 3.5, baseando-se numa função $Q R$ previamente implementada, o algoritmo para a obtenção do complemento ortogonal a partir da matriz Yx é imediato, bastando subdividir as matrizes $Q, R$ da decomposição como em (3.5).

\section{Algoritmo 3.6 (Complemento Ortogonal)}

Entradas do Algoritmo: $H_{n \times s}$

Saídas do Algoritmo: $r, R$

o Aplicando a decomposição e subdividindo conforme observação anterior

$[Q, \tilde{R}] \leftarrow \mathrm{QR}\left(H^{\top}\right) ;\left[\begin{array}{ll}r & 0\end{array}\right] \leftarrow \tilde{R}^{\top} ;\left[\begin{array}{c}q \\ R\end{array}\right] \leftarrow Q^{\top}$ 


\subsection{Coordenadas Complementares via Complemento Ortogonal - $\mathrm{MII}_{\text {proj }}$}

Estuda-se aqui como aplicar o Complemento Ortogonal para o MII, visando a aplicação de um método para resolução numérica de EDOs. Denote-se a função $R: \mathcal{A} \rightarrow \mathbb{R}^{n \times(n-\rho)}$ associando pontos $x \in \mathcal{A}$ ao complemento ortogonal de $Y_{x}$, pela decomposição QR conforme (3.5). Um dos problemas a ser entendido nesta seção é que a decomposição QR não é um processo diferenciável e poderia gerar também um campo $\tau(t, x, u)$ não diferenciavelmente em relação a $x$, já que ele é construído utilizando-se $R(x)$.

não é um processo diferenciável não depender diferenciavelmente de $x$.

Impondo $\frac{\partial \hat{x}}{\partial x}=R(x)$ em cada ponto da discretização do método numérico, o MII será:

$$
\left(\begin{array}{cc}
R(x) & 0 \\
\frac{\partial Y^{(\rho-1)}}{\partial x} & 0 \\
\frac{\partial y^{(\rho)}}{\partial x} & \frac{\partial y^{(\rho)}}{\partial u}
\end{array}\right)\left(\begin{array}{c}
\dot{x} \\
\dot{u}
\end{array}\right)=\left(\begin{array}{c}
R(x)(f(x, u)) \\
-\gamma Y^{(\rho)}(x, u)
\end{array}\right)
$$

Assumindo-se que o sistema acima esteja bem definido, cabe o comentário:

\section{Observação 3.7 (Interpretação Geométrica do Complemento Ortogonal no MII)}

O MII envolve a inversa da matriz $T$ e e por ela ser uma matriz blocada pode-se reescrever (2.55), como:

$$
\left\{\begin{array}{l}
\dot{x}=\tau_{x}(x, u)=\left(\begin{array}{c}
\frac{\partial \hat{x}}{\partial x} \\
\frac{\partial Y^{(\rho-1)}}{\partial x}
\end{array}\right)^{-1}\left(\begin{array}{c}
\frac{\partial \hat{x}}{\partial x} f(x, u) \\
-\gamma Y^{(\rho-1)}
\end{array}\right) \\
\dot{u}=\tau_{u}(x, u)=\left(\frac{\partial y^{(\rho)}}{\partial u}\right)^{-1}\left(-\gamma y^{(\rho)}-\frac{\partial y^{(\rho)}}{\partial x} \dot{x}\right)
\end{array}\right.
$$

Usando por simplicidade $Y_{x}$ para indicar $\frac{\partial Y^{(\rho-1)}}{\partial x}$ e impondo $\frac{\partial \hat{x}}{\partial x}=R(x)$, a matriz inversa do membro direito de (3.7a) no ponto em que foi calculado o complemento ortogonal é dada por

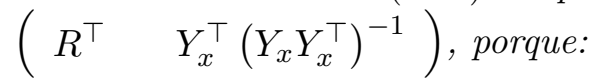

$$
\left(\begin{array}{c}
R \\
Y_{x}
\end{array}\right)\left(\begin{array}{cc}
R^{\top} & Y_{x}^{\top}\left(Y_{x} Y_{x}^{\top}\right)^{-1}
\end{array}\right)=\left(\begin{array}{cc}
R R^{\top} & R Y_{x}^{\top}\left(Y_{x} Y_{x}^{\top}\right)^{-1} \\
Y_{x} R^{\top} & Y_{x} Y_{x}^{\top}\left(Y_{x} Y_{x}^{\top}\right)^{-1}
\end{array}\right)=I_{n \times n}
$$

Visto que $R R^{\top}=I_{\rho \times \rho}$, pois as linhas de $R$ são ortogonais; $R Y_{x}^{\top}=0_{\rho \times(n-\rho)}$ e $Y_{x} R^{\top}=0_{(n-\rho) \times \rho}$, pois $R$ é o complemento ortogonal de $Y_{x}$; e $Y_{x} Y_{x}^{\top}\left(Y_{x} Y_{x}^{\top}\right)^{-1}=I_{(n-\rho \times n-\rho)}$ por definição, salientando que a matriz $\left(Y_{x} Y_{x}^{\top}\right)$ é inversivel já que:

$$
\left(Y_{x} Y_{x}^{\top}\right)=r q(r q)^{\top}=r q q^{\top} r^{\top}=r r^{\top}
$$

e a matriz quadrada $r$ é inversivel, desde que $Y_{x}$ tenha posto máximo como visto na seção anterior. 
Substituindo em (3.7a) a expressão da inversa, tem-se:

$$
\begin{aligned}
& \dot{x}=\left(\begin{array}{c}
R \\
\frac{\partial Y^{(\rho-1)}}{\partial x}
\end{array}\right)^{-1}\left(\begin{array}{c}
R f(x, u) \\
-\gamma Y^{(\rho-1)}
\end{array}\right) \\
& =\left(\begin{array}{cc}
R^{\top} & Y_{x}^{\top}\left(Y_{x} Y_{x}^{\top}\right)^{-1}
\end{array}\right)\left(\begin{array}{c}
R f(x, u) \\
-\gamma Y^{(\rho-1)}
\end{array}\right) \\
& =\underbrace{R^{\top} R}_{n \times n} f(x, u)-\gamma \underbrace{Y_{x}^{\top}\left(Y_{x} Y_{x}^{\top}\right)^{-1}}_{n \times \rho} Y^{(\rho-1)}
\end{aligned}
$$

Com a vantagem de que $Y_{x} Y_{x}^{\top}=r q(r q)^{\top}=r q q^{\top} r^{\top}=r r^{\top}$ e portanto o cálculo de $\left(Y_{x} Y_{x}^{\top}\right)^{-1} Y^{(\rho-1)}$ pode ser feito com duas eliminações de Gauss envolvendo matrizes triangulares de ordem $\rho$.

Além disso, já que os vetores linha de $R$ são ortonormais, o termo $R^{\top} R f(x, u)$ expressa geometricamente a projeção do vetor $f(x, u)$ no espaço gerado pelo vetores linha de $R$, indicado por $[R]=[R(x)]$. Justificando a notação $\operatorname{proj}_{[R]}(f(x, u)) \doteq R^{\top} R f(x, u)$.

Enquanto o termo $Y_{x}^{\top}\left(Y_{x} Y_{x}^{\top}\right)^{-1} Y^{(\rho-1)}(x, u)$ pode ser interpretado como uma inclusão do vetor $Y^{(\rho-1)}(x, u) \in \mathbb{R}^{\rho}$ no subespaço gerado pelos vetores linha de $Y_{x}$ avaliados no ponto $x \in$ $\mathcal{A}$, indicado por $\left[Y_{x}\right]=\left[\left.Y_{x}\right|_{x}\right]$ contido no $\mathbb{R}^{n}$. Justificando a notação $\operatorname{inc}_{\left[Y_{x}\right]}\left(Y^{(\rho-1)}(x, u)\right) \doteq$ $Y_{x}^{\top}\left(Y_{x} Y_{x}^{\top}\right)^{-1} Y^{(\rho-1)}(x, u)$. [Str80].

Observe também que para todo $x \in \mathcal{A},\left[\left.Y_{x}\right|_{x}\right]^{\perp}=[R(x)]$ e, pelo Lema $3.4, \mathbb{R}^{n}=\left[\left.Y_{x}\right|_{x}\right] \oplus[R(x)]$. Com esta imposição de $\frac{\partial \hat{x}}{\partial x}=R(x)$ o sistema (3.7) será denominado $M_{\text {proj }}$ e será igual a:

$$
\left\{\begin{array}{l}
\dot{x}=\tau_{x}(x, u)=\operatorname{proj}_{[R]}(f(x, u))-\gamma \operatorname{inc}_{\left[Y_{x}\right]}\left(Y^{(\rho-1)}(x)\right) \\
\dot{u}=\tau_{u}(x, u)
\end{array}\right.
$$

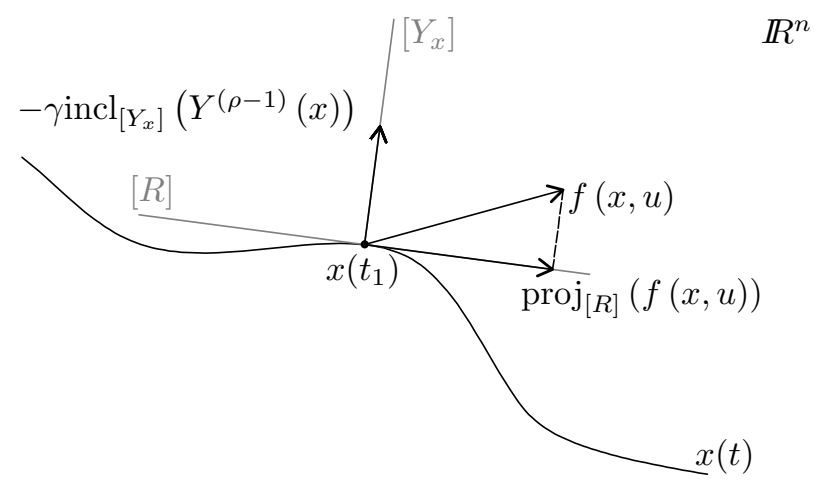

Figura 3.1: Interpretação geométrica das componentes do campo vetorial $\tau_{x}$ do $M I I_{\text {proj }}$.

Chama-se a atenção que a implementação do $M I_{\text {proj }}$ é feita utilizando-se (3.11).

Contudo, mesmo que a expressão do campo $\tau(x, u)$ envolva somente o valor de $\frac{\partial \hat{x}}{\partial x}$, o MII faz sentido somente se a aplicação $\Psi(x, u)=\left(\hat{x}(x), Y^{(\rho)}(x, u)\right)$ for uma transformação de coordenadas diferenciável. No entanto, essa escolha numérica de complemento ortogonal via decomposição QR não garante a princípio nem mesmo a existência de função $\hat{x}$ tal que $\frac{\partial \hat{x}}{\partial x}=R(x)$. Expõem-se abaixo resultados que justificam essa escolha. 
Proposição 3.8 Seja um aberto $\mathcal{W}=\mathcal{U} \times \mathcal{V} \subset \mathcal{S}$ no qual $\frac{\partial y^{(\rho)}}{\partial u}$ possui posto máximo. Então uma função suave $x(t)$ com entrada $u(t)$ e condição inicial $\left(x_{0}, u_{0}\right) \in \Gamma$, definidas para $t \in I$ onde $(x(t), u(t)) \in \mathcal{W}, \forall t \in I$, é solução do Sistema Implicito se e somente se $(x(t), u(t))$ é solução de $M I I_{\text {proj }}$. com condição inicial $\left(x_{0}, u_{0}\right)$.

Prova Como $h(x(t), u(t)) \equiv 0$, vale que $Y^{(\rho)}(x(t), u(t))=0, \forall t \in I$.

Além disso, para todo $i \in\{1, \ldots, m\}$ e $t \in I, 0=h_{i}(x(t), u(t)) \doteq y_{i}^{(0)}((x(t), u(t))$, (vide $(2.23))$, logo:

$$
y_{i}^{(j+1)}(x) \doteq \frac{d}{d t}\left(y_{i}^{(j)}(x)\right)=\frac{\partial y_{i}^{(j)}}{\partial x} \dot{x}=\left\langle\dot{y}_{i}^{(j)}, f(x, u)\right\rangle=0, \text { para todo } j \in\left\{0, \ldots, \rho_{i}-2\right\}
$$

Ou seja, o vetor $f(x(t), u(t)) \in \mathbb{R}^{n}$ é perpendicular ao subespaço $\left[\left.Y_{x}\right|_{x(t))}\right]$.

Portanto $f(x(t), u(t)) \in[R(x(t))] \operatorname{eproj}_{[R]}(f(x(t), u(t)))=(f(x(t), u(t))) \forall t \in I$.

$\mathrm{E}, \dot{x}(t)=f(x(t), u(t))=\operatorname{proj}_{[R]}(f(x(t), u(t)))-\gamma \operatorname{inc}_{\left[Y_{x}\right]}\left(Y^{(\rho-1)}(x(t), u(t))\right)$, para todo $t \in I$.

Para a dinâmica de $u$, usando o Teorema 2.23 já que $\frac{\partial y^{(\rho)}}{\partial u}$ possui posto máximo em $\mathcal{W}$, o fato de $(x(t), u(t))$ ser solução do Sistema Implícito equivale a $(x(t), u(t))$ ser solução do MII para um campo vetorial $\bar{\tau}(x, u)$ como em (2.55). Frisando que as escolhas locais de coordenadas complementares $\hat{x}(x)$ envolvidas na construção do MII não precisam obedecer $\left.\frac{\partial \hat{x}}{\partial x}\right|_{x}=R(x)$.

Denote-se o campo do $\mathrm{MII}_{\text {proj }}$ conforme (3.11) por $\tau(x, u)$.

Pelo Observação 2.24 Item $2, \bar{\tau}(x, u)$ não depende da escolha de $\hat{x}(x)$, e como visto acima, $\tau_{x}(x, u)$ também não depende da escolha particular da base ortonormal para $[R(x)]$.

Portanto:

$$
\begin{aligned}
\tau_{u}(x, u) & =\left(\frac{\partial y^{(\rho)}}{\partial u}\right)^{-1}\left(-\gamma y^{(\rho)}-\frac{\partial y^{(\rho)}}{\partial x} \dot{x}\right) \\
& =\left(\frac{\partial y^{(\rho)}}{\partial u}\right)^{-1}\left(-\gamma y^{(\rho)}-\frac{\partial y^{(\rho)}}{\partial x} f(x, u)\right) \\
& =\bar{\tau}_{u}(x, u)
\end{aligned}
$$

Concluindo-se a primeira parte do teorema. Para a recíproca, vale o raciocínio análogo.

Outro aspecto essencial é se o $\mathrm{MII}_{\text {proj }}$ está bem definido para condições iniciais $\left(x_{0}, u_{0}\right) \in \Gamma_{\varepsilon}$ $\operatorname{com} \varepsilon>0$ pequeno. Para tanto a observação abaixo mostra que ele na verdade pode ser construindo a partir de uma escolha qualquer de coordenadas complementares $\hat{x}(x)$.

Observação 3.9 Seja $\left(x_{0}, u_{0}\right) \in \Gamma_{\varepsilon}$, com $\varepsilon>0$ pequeno; $\mathcal{W}=\mathcal{U} \times \mathcal{V} \subset \mathcal{S}$ uma vizinhança de $\left(x_{0}, u_{0}\right)$ na qual $\frac{\partial y^{(\rho)}}{\partial u}$ tem posto máximo; e uma escolha de coordenadas complementares qualquer $\hat{x}: \mathcal{U} \rightarrow \mathbb{R}^{n-\rho}$.

Escrevendo a primeira equação do MII para esta escolha de $\hat{x}$, conforme (3.7a), tem-se:

$$
\left(\begin{array}{c}
\frac{\partial \hat{x}}{\partial x} \\
\frac{\partial Y^{(\rho-1)}}{\partial x}
\end{array}\right) \dot{x}=\left(\begin{array}{c}
\frac{\partial \hat{x}}{\partial x} f(x, u) \\
-\gamma Y^{(\rho-1)}(x, u)
\end{array}\right)
$$

Defina-se a função $M: \mathcal{U} \rightarrow \mathbb{R}^{n \times n}$, satisfazendo:

$$
M(x)\left(\begin{array}{c}
\left.\frac{\partial \hat{x}}{\partial x}\right|_{x} \\
\left.\frac{\partial Y^{(\rho-1)}}{\partial x}\right|_{x}
\end{array}\right)=\left(\begin{array}{c}
R(x) \\
\left.\frac{\partial Y^{(\rho-1)}}{\partial x}\right|_{x}
\end{array}\right)
$$


Note que $M(x)$ está bem definida e é não singular para todo $x \in \mathcal{U}$. Em particular, $M(x)^{-1} M(x)=$ $I_{n \times n}$ para todo $x \in \mathcal{U}$.

$$
\begin{aligned}
& \text { Assim, denotando-se } \frac{\partial Y^{(\rho-1)}}{\partial x} \text { por } Y_{x} \text { e } \frac{\partial \hat{x}}{\partial x} \text { por } \hat{x}_{x} \text {, obtém-se: } \\
& \left(\begin{array}{c}
\hat{x}_{x} \\
Y_{x}
\end{array}\right) \dot{x}=\left(\begin{array}{c}
\hat{x}_{x} f(x, u) \\
-\gamma Y^{(\rho-1)}(x, u)
\end{array}\right) \\
& \Leftrightarrow M(x)\left(\begin{array}{c}
\hat{x}_{x} \\
Y_{x}
\end{array}\right) \dot{x}=M(x)\left(\begin{array}{c}
\hat{x}_{x} f(x, u) \\
-\gamma Y^{(\rho-1)}(x, u)
\end{array}\right) \\
& \Leftrightarrow \quad\left(\begin{array}{c}
R(x) \\
Y_{x}
\end{array}\right) \dot{x}=\left(\begin{array}{c}
R(x) f(x, u) \\
-\gamma M(x) Y^{(\rho-1)}
\end{array}\right) \text {, usando a seguir (3.8) } \\
& \Leftrightarrow \quad \dot{x}=R(x)^{\top} R(x)(f(x, u))-\gamma\left(Y_{x}\right)^{\top}\left(Y_{x} Y_{x}^{\top}\right)^{-1} M(x) Y^{(\rho-1)}(x, u) \\
& \Leftrightarrow \quad \dot{x}=\operatorname{proj}_{[R(x)]}(f(x, u))-\gamma \operatorname{inc}_{\left[Y_{x}\right]}\left(Y^{(\rho-1)}(x, u)\right)
\end{aligned}
$$

Notando que $M(x) Y_{x}=Y_{x}$ em decorrência de (3.14) e utilizando na última equivalência que:

$$
\begin{aligned}
\left(Y_{x}\right)^{\top}\left(Y_{x} Y_{x}^{\top}\right)^{-1} M(x) Y^{(\rho-1)} & =\left(Y_{x}\right)^{\top} M(x)^{\top}\left(M(x) Y_{x} Y_{x}^{\top} M(x)^{\top}\right)^{-1} M(x) Y^{(\rho-1)} \\
& =\left(Y_{x}\right)^{\top} M(x)^{\top}\left(M(x)^{\top}\right)^{-1}\left(Y_{x} Y_{x}^{\top}\right)^{-1} M(x)^{-1} M(x) Y^{(\rho-1)} \\
& =\left(Y_{x}\right)^{\top}\left(Y_{x} Y_{x}^{\top}\right)^{-1} Y^{(\rho-1)} \\
& =\operatorname{inc}_{\left[Y_{x}\right]}\left(Y^{(\rho-1)}\right)
\end{aligned}
$$

Portanto os teoremas da Seção 2.6 também são válidos para o $M I I_{\text {proj }}$.

Os algoritmos para essa escolha de coordenadas complementares, bem como outras discussões sobre a aplicação do método, são apresentados na Seção 3.6.

\subsection{Coordenadas Complementares via Preservação de Condiciona- mento - MII $_{\text {cond }}$}

Analisa-se nesta seção uma outra alternativa para obtenção de coordenadas complementares $\hat{x}$ numa vizinhança de um ponto $x_{0} \in \mathcal{A}$.

A partir da Observação 2.18, desde que o posto de $Y_{x} \doteq \frac{\partial Y^{(\rho-1)}}{\partial x}$ seja máximo, pode-se realizar uma escolha local de coordenadas complementares local da forma $\hat{x}(x)=K x$, onde $K$ é uma matriz fixa $n \times(n-\rho)$ tal que $\left(\begin{array}{c}K \\ Y_{x}\end{array}\right)$ seja não singular em $x_{0}$.

Ora, o complemento ortogonal de $\left.Y_{x}\right|_{x_{0}}$ satisfaz essa condição e portanto pode-se tomar $\hat{x}(x)=$ $\mathcal{U} \rightarrow \mathbb{R}^{n-\rho}$ definida por $\hat{x}(x)=R\left(x_{0}\right) x$, para alguma vizinhança $\mathcal{U}$ de $x_{0}$.

Todavia, diferentemente da seção anterior, a inversa da matriz $\left(\begin{array}{c}R\left(x_{0}\right) \\ Y_{x}\end{array}\right)$ não cumpre (3.8), pois $R\left(x_{0}\right)\left(\left.Y_{x}\right|_{x_{1}}\right)^{\top}$ não é necessariamente nula para $x_{0} \neq x_{1} \in \mathcal{U}$.

Outrossim, é possível buscar uma transformação de coordenadas sobre $Y^{(\rho)}$ obtendo $\tilde{Y}^{(\rho)}=$ $C Y^{(\rho)}$, para alguma matriz fixa $C_{(n-\rho+m) \times(n-\rho+m)}$ não singular, de modo a facilitar a inversão da matriz $\tilde{T}(x, u)=\frac{\partial \tilde{\Psi}}{\partial x, u}$, com $\tilde{\Psi}(x, u)=\left(\hat{x}(x), \tilde{Y}^{(\rho)}(x, u)\right)$, para $(x, u)$ suficientemente próximos de $\left(x_{0}, u_{0}\right)$, como será discutido em seguida. 
Observação 3.10 (Transformação de coordenadas sobre $Y^{(\rho-1)}$ )

Considere-se o MII dado por (2.55), substituindo $Y^{(\rho-1)}(x)$ por $\tilde{Y}^{(\rho-1)}(x)=C Y^{(\rho-1)}(x)$ e $y^{(\rho)}(x, u)$ por $\tilde{y}^{(\rho)}(x, u)=D y^{(\rho)}(x, u)$, para matrizes fixas não singulares de $C_{(n-\rho) \times(n-\rho)}$ e $D_{m \times m}$. Logo, as seguintes equivalências são imediatas:

$$
\begin{aligned}
&\left(\begin{array}{cc}
\frac{\partial \hat{x}}{\partial x} & 0 \\
\frac{\partial \tilde{Y}^{(\rho-1)}}{\partial x} & 0 \\
\frac{\partial \tilde{y}^{(\rho)}}{\partial x} & \frac{\partial \tilde{y}^{(\rho)}}{\partial u}
\end{array}\right)\left(\begin{array}{c}
\dot{x} \\
\dot{u}
\end{array}\right)=\left(\begin{array}{c}
\frac{\partial \hat{x}}{\partial x} f \\
-\gamma \tilde{Y}^{(\rho-1)} \\
-\gamma \tilde{y}^{(\rho)}
\end{array}\right) \\
& \Leftrightarrow \quad\left(\begin{array}{lll}
I & 0 & 0 \\
0 & C & 0 \\
0 & 0 & D
\end{array}\right)\left(\begin{array}{cc}
\frac{\partial \hat{x}}{\partial x} & 0 \\
\frac{\partial Y^{(\rho-1)}}{\partial x} & 0 \\
\frac{\partial y^{(\rho)}}{\partial x} & \frac{\partial y^{(\rho)}}{\partial u}
\end{array}\right)\left(\begin{array}{c}
\dot{x} \\
\dot{u}
\end{array}\right)=\left(\begin{array}{ccc}
I & 0 & 0 \\
0 & C & 0 \\
0 & 0 & D
\end{array}\right)\left(\begin{array}{c}
\frac{\partial \hat{x}}{\partial x} f \\
-\gamma Y^{(\rho-1)} \\
-\gamma y^{(\rho)}
\end{array}\right) \\
& \Leftrightarrow \quad\left(\begin{array}{cc}
\frac{\partial \hat{x}}{\partial x} & 0 \\
\frac{\partial Y^{(\rho-1)}}{\partial x} & 0 \\
\frac{\partial y^{(\rho)}}{\partial x} & \frac{\partial y^{(\rho)}}{\partial u}
\end{array}\right)\left(\begin{array}{l}
\dot{x} \\
\dot{u}
\end{array}\right)=\left(\begin{array}{c}
\frac{\partial \hat{x}}{\partial x} f \\
-\gamma Y^{(\rho-1)} \\
-\gamma y^{(\rho)}
\end{array}\right)
\end{aligned}
$$

Logo, os teoremas da Seção 2.6 também são válidos para esta modificação do MII.

As matrizes $C$ e $D$ da observação anterior podem ser interpretadas como balanceamentos. Esses balanceamentos serão escolhidos com auxílio da decomposição QR, para que no ponto onde elas forem calculadas os blocos na diagonal de $T(x, u)$ sejam matrizes ortogonais.

\section{Observação 3.11 (Escolha de Balanceamento)}

Para um ponto $\left(x_{0}, u_{0}\right)$ podem-se tomar $C=r_{1}^{-1}$ e $D=r_{2}^{-1}$, onde as matrizes $r_{1}$ e $r_{2}$ são tais que $r_{1} q_{1}=\left.\frac{\partial Y^{(\rho-1)}}{\partial x}\right|_{x_{0}} e r_{2} q_{2}=\left.\frac{\partial y^{(\rho)}}{\partial u}\right|_{\left(x_{0}, u_{0}\right)}$, com notação análoga à (3.5).

Observando que os cálculos $\tilde{Y}^{(\rho-1)}=r_{1}^{-1} Y^{(\rho-1)}$ e $\tilde{y}^{(\rho)}=r_{2}^{-1} y^{(\rho)}$ podem ser feitos por eliminação de Gauss envolvendo as matrizes triangulares $r_{1}, r_{2}$.

Daí, numa vizinhança $\mathcal{V}$ da condição inicial $\left(x_{0}, u_{0}\right)$ o MII será da forma:

$$
\left(\begin{array}{cc}
R\left(x_{0}\right) & 0 \\
\frac{\partial \tilde{Y}^{(\rho-1)}}{\partial x} & 0 \\
\frac{\partial \tilde{y}^{(\rho)}}{\partial x} & \frac{\partial \tilde{y}^{(\rho)}}{\partial u}
\end{array}\right)\left(\begin{array}{l}
\dot{x} \\
\dot{u}
\end{array}\right)=\left(\begin{array}{c}
R\left(x_{0}\right) f(x, u) \\
-\gamma \tilde{Y}^{(\rho-1)} \\
-\gamma \tilde{y}^{(\rho)}
\end{array}\right)
$$

Onde $\left.\frac{\partial \tilde{Y}^{(\rho-1)}}{\partial x}\right|_{x_{0}}=r_{1}^{-1}\left(\left.\frac{\partial Y^{(\rho-1)}}{\partial x}\right|_{x_{0}}\right)=\left.q_{1} \quad e \frac{\partial \tilde{y}^{(\rho)}}{\partial(x, u)}\right|_{\left(x_{0}, u_{0}\right)}=r_{2}^{-1}\left(\left.\frac{\partial y^{(\rho)}}{\partial(x, u)}\right|_{\left(x_{0}, u_{0}\right)}\right)=q_{2}$ por construção. Assim, pela continuidade da derivada de $Y^{(\rho)}(x, u)$ em $\mathcal{V}$, os blocos na diagonal da matriz $T(x, u)$, no membro esquerdo de (3.16), estarão próximos das matrizes ortogonais $\left(\begin{array}{c}R\left(x_{0}\right) \\ q_{1}\end{array}\right)$ e $q_{2}$, desde que $(x, u)$ esteja próximo de $\left(x_{0}, u_{0}\right)$.

Conclui-se então que solução do MII está bem definida para $t \in\left[0, t_{1}\right)$, desde que $(x(t), u(t)) \in \mathcal{U}$. Entretanto, com o discutido até aqui ainda não se definiu como proceder quando os blocos da diagonal de $T(x, u)$ se "afastam da ortogonalidade", prejudicando a acuidade numérica do método, ou como computar as soluções $(x(t), u(t))$ para $t \geq t_{1}$.

Para lidar com essas duas questões, lança-se mão do conceito de condicionamento para uma matriz real, por [Str80]. 
Lema 3.12 Se A é uma matriz real $m \times n$, então existe uma decomposição $A=U S V^{\top}$ onde $U_{m \times m}$ $e V_{n \times n}$ são matrizes ortogonais e $S_{m \times n}$ é uma matriz diagonal. Os elementos na diagonal de $V$ são não negativos e ordenados de forma decrescente, recebendo o nome de valores singulares de A.

Definição 3.13 Se $A$ é uma matriz real $m \times n$, a razão $\operatorname{cond}(A)$ entre a norma do maior e do menor valor singular de $A$ recebe o nome de número de condicionamento de $A$.

O condicionamento de uma matriz $A$ não singular é importante por representar, independente do método numérico utilizado, uma estimativa da precisão para a obtenção numérica da solução $x$ do sistema linear $A x=b$.

Observando que o melhor condicionamento possível é $\operatorname{cond}(A)=1$, atingido para matrizes $A$ ortogonais. Enquanto que se $A$ for singular, ao menos um de seus valores singulares será nulo, indicando-se por abuso de notação $\operatorname{cond}(A)=\infty$.

Voltando ao MII com a escolha $\hat{x}(x)=R\left(x_{0}\right) x$, é possível monitorar o pior condicionamento dentre os blocos na diagonal de $T(x, u)$, computando:

$$
K(t) \doteq \max \left\{\operatorname{cond}\left(\begin{array}{c}
R\left(x_{0}\right) \\
\left.\frac{\partial Y^{(\rho-1)}}{\partial x}\right|_{x(t)}
\end{array}\right) \text {, cond }\left(\left.\frac{\partial y^{(\rho)}}{\partial(x, u)}\right|_{(x(t), u(t))}\right)\right\}
$$

Assim, pode-se fixar uma tolerância para o condicionamento tol cond $>1$ e se $K(t)>$ tol $_{\text {cond }}$ para algum instante $t_{1}$, aplica-se novamente o MII, desta vez com condição inicial $\left(x_{1}, u_{1}\right) \doteq\left(x\left(t_{1}\right), u\left(t_{1}\right)\right)$ e $\hat{x}(x)=R\left(x_{1}\right) x$, calculando-se novas matrizes de balanceamento $C_{1}$ e $D_{1}$ pela Observação 3.11 .

O MII com essa escolha de coordenadas complementares, preservando o condicionamento de $T(x, u)$ como discutido acima, será denotado por $\mathrm{MII}_{\text {cond }}$.

O algoritmos para essa escolha de coordenadas complementares, bem como outras discussões sobre a aplicação do método, são apresentados na Seção 3.6.

\subsection{Sistemas Variantes no Tempo}

Considere um sistema semi-implícito quadrado variante no tempo:

$$
\left\{\begin{array}{l}
\dot{x}=f(t, x, u) \\
y=h(t, x, u) \equiv 0
\end{array}\right.
$$

Onde $f: I \times \mathcal{A} \times \mathbb{R}^{m} \rightarrow \mathbb{R}^{n}$ e $h: I \times \mathcal{A} \times \mathbb{R}^{m} \rightarrow \mathbb{R}^{m}$ são funções suaves, tal como (2.48), acrescentando-se a dependência em relação ao tempo nas funções $f$ e $h$ para $t \in I$, um intervalo de $\mathbb{R}$.

Aqui será utilizado o artifício comum de inserir a variável $t$ no estado do sistema, impondo-se $\dot{t}=1$ com condição inicial $t(0)=t_{0}$. A transformação de coordenadas tomada será $\Psi(t, x, u)=$ $\left(t, \hat{x}(t, x), Y^{(\rho-1)}(t, x), y^{(\rho)}(t, x, u)\right)$, para alguma escolha de coordenadas complementares $\hat{x}(t, x)$ (como na Proposição 2.17) e para funções $Y^{(\rho-1)}(t, x)$ e $y^{(\rho)}(t, x, u)$ obtidas através do Algoritmo de Derivações Sucessivas 3.2 considerando o campo vetorial $\left(\begin{array}{c}1 \\ f(t, x, u)\end{array}\right)$.

Mantendo a notação $T(t, x, u) \doteq \frac{\partial \Psi(t, x,)}{\partial(t, x, u)}$, o MII será:

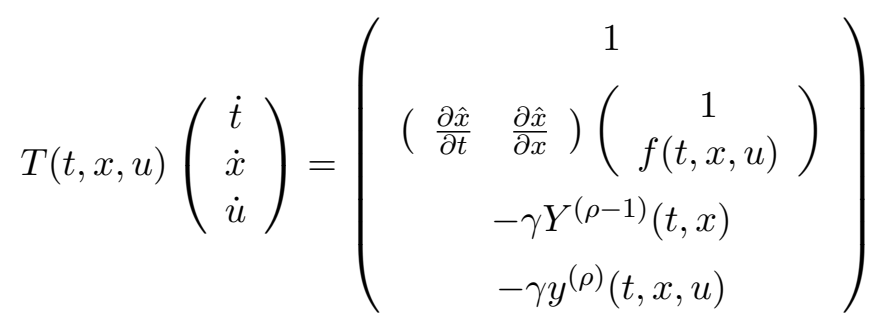


Onde a matriz $T(t, x, u)$ é da forma:

$$
T(t, x, u)=\left(\begin{array}{ccc}
1 & 0 & 0 \\
\frac{\partial \hat{x}}{\partial t} & \frac{\partial \hat{x}}{\partial x} & 0 \\
\frac{\partial Y^{(\rho-1)}}{\partial t} & \frac{\partial Y^{(\rho-1)}}{\partial x} & 0 \\
\frac{\partial y^{(\rho)}}{\partial t} & \frac{\partial y^{(\rho)}}{\partial x} & \frac{\partial y^{(\rho)}}{\partial u}
\end{array}\right)
$$

Daí, observa-se que se pode escolher $\hat{x}(t, x)=\hat{x}(x)$, i.e., como função somente do estado $x$ do sistema, pois a imposição de $\frac{\partial \hat{x}}{\partial t} \equiv 0$ não modifica o posto de $T(t, x, u)$.

Assim, o sistema (3.19) torna-se:

$$
\left(\begin{array}{cc}
0 & 0 \\
\frac{\partial \hat{x}}{\partial x} & 0 \\
\frac{\partial Y^{(\rho-1)}}{\partial x} & 0 \\
\frac{\partial y^{(\rho)}}{\partial x} & \frac{\partial y^{(\rho)}}{\partial u}
\end{array}\right)\left(\begin{array}{c}
\dot{x} \\
\dot{u}
\end{array}\right)+\left(\begin{array}{c}
1 \\
0 \\
\frac{\partial Y^{(\rho-1)}}{\partial t} \\
\frac{\partial y^{(\rho)}}{\partial t}
\end{array}\right) \dot{t}=\left(\begin{array}{c}
1 \\
\frac{\partial \hat{x}}{\partial x} f(t, x, u) \\
-\gamma Y^{(\rho-1)}(t, x) \\
-\gamma y^{(\rho)}(t, x, u)
\end{array}\right)
$$

Ademais, como a evolução no tempo é controlada internamente pelo método numérico, a equação $\dot{t}=1$ pode ser removida, resultando:

$$
\left(\begin{array}{cc}
\frac{\partial \hat{x}}{\partial x} & 0 \\
\frac{\partial Y^{(\rho-1)}}{\partial x} & 0 \\
\frac{\partial y^{(\rho)}}{\partial x} & \frac{\partial y^{(\rho)}}{\partial u}
\end{array}\right)\left(\begin{array}{c}
\dot{x} \\
\dot{u}
\end{array}\right)=\left(\begin{array}{c}
\frac{\partial \hat{x}}{\partial x} f(t, x, u) \\
-\gamma Y^{(\rho-1)}(t, x) \\
-\gamma y^{(\rho)}(t, x, u)
\end{array}\right)-\left(\begin{array}{c}
0 \\
\frac{\partial Y^{(\rho-1)}}{\partial t} \\
\frac{\partial y^{(\rho)}}{\partial t}
\end{array}\right)
$$

Ressaltando que esta técnica pode ser utilizada sem custo adicional para o método de resolução numérica de EDOs mesmo para sistemas autônomos, já que neles o termo mais à direita de (3.22) será nulo, obtendo-se a mesma expressão MII em (2.55).

\subsection{Construção da Implementação}

Os algoritmos desta seção têm como intuito organizar as ideias expostas até aqui. Os códigosfonte podem ser encontrados na Seção 6.1.

Descreve-se agora a construção do algoritmo parametrizado por:

- funções $f, h$, nas variáveis $t, x, u$, na estrutura (3.18)

- uma variável Tipo_de_Simulação, igual a 1 para MII $_{\text {proj }}\left(\right.$ Seção 3.3) ou 2 para o MII cond $_{\text {(Seção }}$ $3.4)$

- um parâmetro de convergência gama> 0 (Seção 2.5)

- para o $\mathrm{MII}_{\text {cond }}$, uma tolerância para o condicionamento tol $\mathrm{l}_{\text {cond }}>1$ (Seção 3.4)

As etapas descritas a seguir são as mesmas da Seção 1.1.

\section{Etapa 1: Cálculos Simbólicos}

O algoritmo descrito abaixo utiliza o Algoritmo 3.2, possibilitando a generalização do MII para sistemas variantes no tempo da Seção 3.5.

\section{Algoritmo 3.14 (ConstroiImplementacao)}




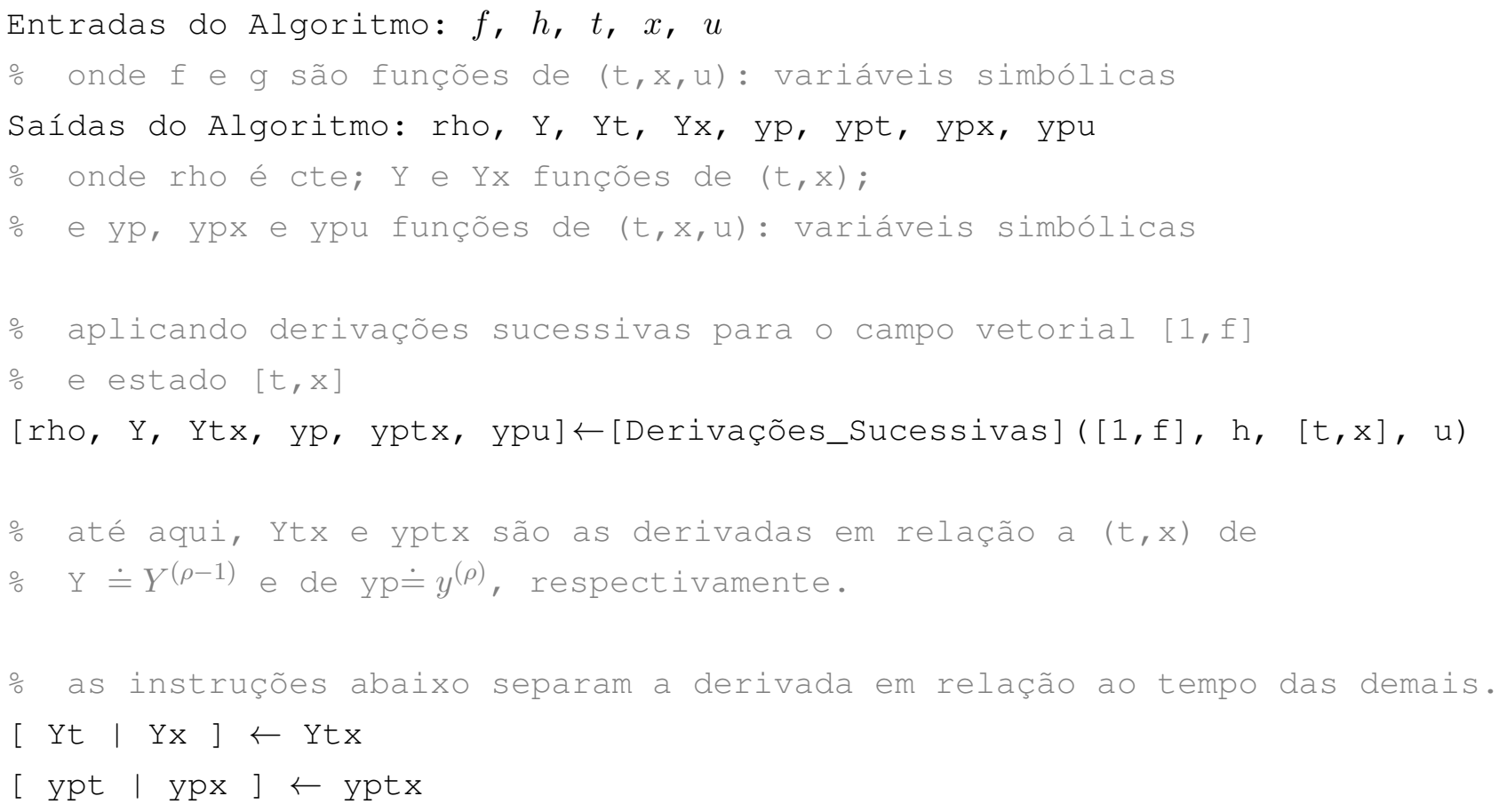

\section{Etapas 2 e 3: Cálculos Numéricos}

Para a integração numérica do MII, é utilizado o seguinte diagrama.

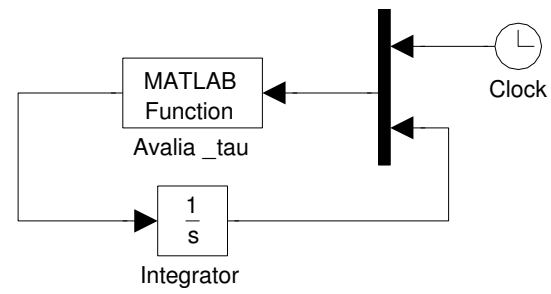

Figura 3.2: Diagrama de simulação, padrão Matlab.

A avaliação do campo $\tau$ no ponto $(t, x, u)$ (vide 3.22 ), nas versões $M I I_{\text {proj }}$ ou $M I I_{\text {cond }}$, utiliza as variáveis simbólicas resultantes do algoritmo anterior é feita por:

\section{Algoritmo 3.15 (Avalia_tau)}

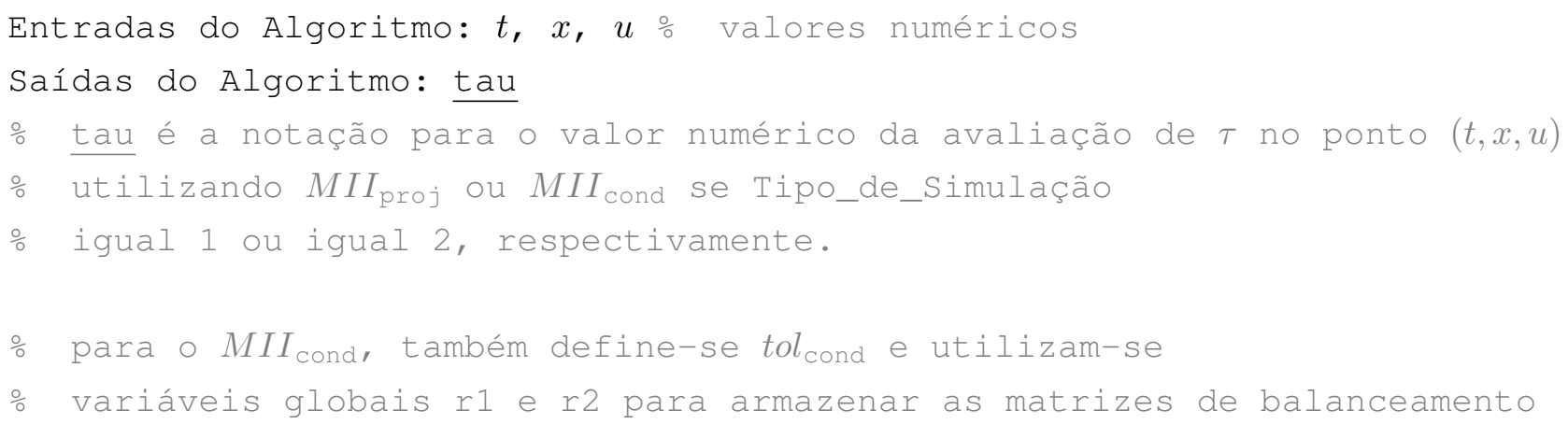


\% avaliando as funções necessárias no ponto $(t, x, u)$

o a barra abaixo dos símbolos indica que a variável é do tipo numérica

txu $\leftarrow(t, x, u) ;$

$\underline{f} \leftarrow f(t x u)$

$\underline{\mathrm{Y}} \leftarrow \mathrm{Y}(\mathrm{txu})$

$\underline{y p} \leftarrow y p(t x u) ;$

$\underline{\mathrm{Yt}} \leftarrow \mathrm{Yt}(\mathrm{txu}) ; \underline{\mathrm{Yx}} \leftarrow \mathrm{Yx}(\mathrm{txu}) ;$

$\underline{\text { ypt }} \leftarrow \operatorname{ypt}(t x u) ; \underline{\operatorname{ypx}} \leftarrow \operatorname{ypx}(t x u) ; \underline{\text { ypu }} \leftarrow \operatorname{ypu}(t x u) ;$

\% a notação $\mathrm{A} \backslash$ b abaixo indica o processo de eliminação de

\% Gauss para obter $\mathrm{x}$ tal que $\mathrm{Ax}=\mathrm{b}$

Se Tipo_de_Simulação $=1 \frac{0}{0}: M I_{\text {proj }}$

$[R, r 1] \leftarrow$ Coordenadas_Complementares $(\underline{\mathrm{Yx}}) ;$ \% algoritmo 3.6

$\operatorname{tau} 1 \leftarrow \mathrm{R}^{\top} * \mathrm{R} * \underline{\mathrm{f}}-\underline{\mathrm{Yx}}^{\top} *\left(\left(\mathrm{r} 1 * \mathrm{r} 1^{\top}\right) \backslash(\underline{\operatorname{gama}} * \underline{\mathrm{Y}}+\underline{\mathrm{Yt}})\right)$

$\operatorname{tau} 2 \leftarrow \underline{-y p u} \backslash(\underline{y p x} * \underline{\operatorname{tau} 1}+$ gama $* \underline{\text { yp}}+\underline{\text { ypt }})$

Se Tipo_de_Simulação $=2$ : MII $_{\text {cond }}$

- Aplicando o balanceamento nas coordenadas,

\% mantendo o mesmo nome das variáveis.

$\underline{\mathrm{Y}} \leftarrow r 1 \backslash \underline{\mathrm{Y}}$

$\underline{\mathrm{yp}} \leftarrow r 2 \backslash \underline{\mathrm{yp}}$

$\underline{\mathrm{Yt}} \leftarrow \mathrm{r} 1 \backslash \underline{\mathrm{Yt}} ; \underline{\mathrm{Yx}} \leftarrow \mathrm{r} 1 \backslash \underline{\mathrm{Yx}} ;$

$\underline{\text { ypt }} \leftarrow r 2 \backslash \underline{\text { ypt }} ; \underline{\text { ypx }} \leftarrow r 2 \backslash \underline{\text { ypx }} ; \underline{\text { ypu }} \leftarrow r 2 \backslash \underline{\text { ypu }} ;$

- Calculando o condicionamento dos blocos diagonais

condRYx $\leftarrow \operatorname{cond}([R, \underline{Y x}]) ; \operatorname{condypu} \leftarrow \operatorname{cond}(\underline{\mathrm{ypu}})$;

Se condRYx $>$ tol $_{\text {cond }}$ ou condYPu $>$ tol $_{\text {cond }}$

\% Se o condicionamento é maior que tol cond

o é preciso recalcular os balanceamentos r1, r2

$[R 1, r 1] \leftarrow$ Coordenadas_Complementares $(\underline{\mathrm{Yx}}) ; \%$ RI não é utilizado

[Q2, r2] $\leftarrow \mathrm{QR}(\underline{\mathrm{ypu}}) ;$

$r 2 \leftarrow r 2^{\top}$

․ R2 pode ser visto como uma matriz de dimensão $0 \times 0$,

o já que ypu é quadrada 


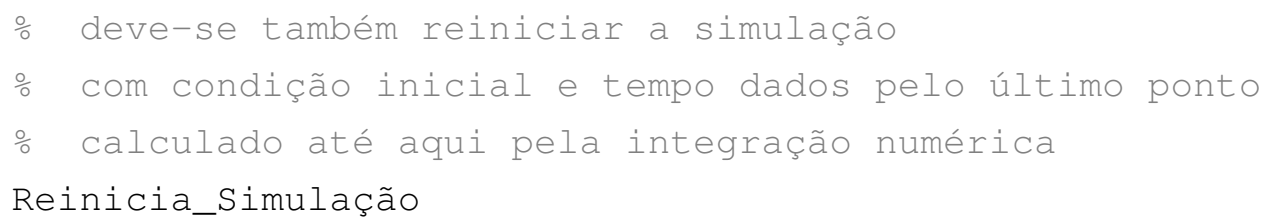

Fim do Se

$\operatorname{tau} 1 \leftarrow\left[\begin{array}{c}\mathrm{R} \\ \underline{\mathrm{Yx}}\end{array}\right] \backslash\left[\begin{array}{c}\mathrm{R} * \underline{\mathrm{f}} \\ - \text { gama } * \underline{\mathrm{Y}}-\underline{\mathrm{Yt}}\end{array}\right]$

$\operatorname{tau} 2 \leftarrow \underline{\text { ypu }} \backslash(-\underline{y p x} *$ taul - gama * $\underline{\text { yp }}-\underline{\text { ypt }})$

Fim do Se

$\underline{\operatorname{tau}} \leftarrow[\tan 1, \tan 2]$

Observação 3.16 Algumas observações importantes sobre o algoritmo anterior:

- Antes da primeira iteração do Algoritmo 3.15 para o $M I I_{\text {cond }}$, já estão calculadas as matrizes r1 e r2 no ponto $\left(x\left(t_{0}\right), u\left(t_{0}\right)\right)$.

- Caso a matriz $T(x, u)$ seja singular em algum ponto durante a simulação, o Algoritmo 3.15 causará um erro e a simulação é finalizada. 


\section{Capítulo 4}

\section{Integração Numérica}

Estuda-se aqui o uso de métodos numéricos para integração numérica dos Sistemas Explícitos $\mathrm{MII}_{\text {proj }}$ e $\mathrm{MII}_{\text {cond }}$, resultantes dos cálculos simbólicos e escolhas numéricas discutidas no capítulo precedente.

A estrutura dos problemas e as características das simulações utilizadas seguem o Test Set for Initial Value Problem Sovers, [MM08] ${ }^{1}$, consistindo em problemas considerados difíceis ou importantes na literatura, comumente chamados de benchmarks no contexto de computação, bem como procedimentos para aferição da acuidade dos métodos numéricos aplicáveis a eles.

São realizadas simulações para sete problemas, brevemente descritos em cada seção, utilizando o Matlab/Simulink ${ }^{\circledR}$. Os três primeiros problemas destinam-se a ilustrar a teoria, enquanto os quatro últimos vêm do [MM08].

Os experimentos numéricos realizados têm como objetivo prático: avaliar o erro da solução numérica contra a solução exata; medir o erro da solução em relação às restrições algébricas; investigar o efeito da variação do parâmetro de convergência $\gamma$ na solução; explorar diferentes métodos de integração numérica e tamanho de passo; e, para os benchmarks, comparar a acuidade do MII com a de outros métodos, através de informações extraídas diretamente do [MM08].

\subsection{Estrutura dos Problemas e Características da Simulação}

Os problemas simulados são sistemas de equações diferenciais não lineares semi-implícitos variantes no tempo, conforme (3.18), na seguinte estrutura:

$$
\left\{\begin{array}{l}
\dot{x}=f(t, x, u) \\
y=h(t, x, u) \equiv 0
\end{array}\right.
$$

Com condição inicial $\left(x\left(t_{0}\right), u\left(t_{0}\right)\right)=\left(x_{0}, u_{0}\right)$, para os instantes $t \in I=\left[t_{0}, t_{f}\right]$. Além disso, é fornecida uma solução de referência $\left(x_{\text {ref }}, u_{\text {ref }}\right)$ aproximando suficientemente $\left(x\left(t_{f}\right), u\left(t_{f}\right)\right)$, para estimar a precisão da solução numérica no instante $t_{f}$.

Além do MII foram incluídos neste trabalho, juntamente com os benchmarks, os resultados apresentados no [MM08] para outros métodos numéricos, cabendo os comentários:

- Os problemas do [MM08] são apresentados originalmente da forma:

$$
M(t, w) \frac{d}{d t}(w)=F(t, w) ; \quad w\left(t_{0}\right)=w_{0} ; \quad w^{\prime}\left(t_{0}\right)=w_{0}^{\prime} ;
$$

Onde $M$ e $F$ dependem suavemente das variáveis $(t, w)$. Observa-se que para a aplicação do MII foi necessário reescrever os problemas no formato semi-implícito, o que é possível somente se $M$ é uma matriz constante.

\footnotetext{
${ }^{1}$ http://pitagora.dm.uniba.it/ ${ }^{\text {testset/ }}$
} 
- Os demais solveres do [MM08] utilizam somente cálculos numéricos, ao contrário do MII que requer a expressão do campo $f$ e da restrição algébrica $h$ para realização das derivações sucessivas no Algoritmo 3.14. Por outro lado, eles exigem uma aproximação para a jacobiana de $F(t, w)$ (que também é fornecida previamente com os problemas), o que não é necessário para o MII, justamente pela utilização dos cálculos simbólicos.

- Não foram simulados 5 dos 9 benchmarks em razão dos itens anteriores.

- Os outros solveres foram implementados na linguagem Fortran, enquanto o MII foi desenvolvido em Matlab, impossibilitando uma comparação justa entre o tempo de processamento já que a primeira linguagem é compilada enquanto a segunda é interpretada, e portanto consideravelmente mais lenta.

- Todos solveres utilizados nas comparações também são de domínio público.

A descrição dos solveres presentes no [MM08] foge ao escopo deste trabalho. Registram-se abaixo somente informações gerais acerca dos solveres pertinentes:

Nome do Solver: $\quad$ BIMD

Autores: $\quad$ C. Magherini and L. Brugnano

Primeira Versão: Outubro, 2005

Última Modificação: Setembro, 2006

Linguagem: $\quad$ Fortran 77

Link oficial: $\quad$ http://www.math.unifi.it/ $\quad$ brugnano/BiM/index.html

Tipos de Problemas: ODEs, DAEs de index menores ou iguais a 3

Breve Descrição: O código BIMD é baseado numa combinação de métodos implícitos de ordens 4, 6, 8, 10 e 12. Ele pertence à classe de métodos implícitos L-estáveis em bloco, definidos como uma combinação adequada de dois métodos equivalentes de maneira a melhorar requisitos de implementação.

Nome do Solver:

DDASS

Autores:

L. Petzold

Primeira Versão:

Março, 1983

Última Modificação:

Julho, 2000

Linguagem:

Fortran 77

Link oficial:

http://www.netlib.org/ode/ddassl.f

Tipos de Problemas: DAEs e Equações Diferenciais Implícitas (IDE) de index 1.

Breve Descrição: Este código implementa um Backward Differentiation Formula de ordens entre 1 e 5 para resolver uma IDE para $y$ e $y^{\prime}$. Valores de $y$ e $y^{\prime}$ no instante inicial devem ser fornecidos como entrada. Estes valores precisam ser consistentes, i.e., $t_{0}, y_{0}, y_{0}^{\prime}$ precisam satisfazer $f\left(t_{0}, y_{0}, y_{0}^{\prime}\right)=0$.

Nome do Solver:

GAMD

Autores:

F. Iavernaro and F. Mazzia

Primeira Versão:

Agosto, 1997

Última Modificação:

Fevereiro, 2006

Linguagem:

Fortran 90

Link oficial: $\quad$ http://www.dm.uniba.it/ $\quad$ mazzia/ode/readme.html

Tipos de Problemas: ODEs e DAEs de index menores do que 3.

Breve Descrição: O código GAMD usa um método generalizado de Adams na forma de bloco, de ordens 3, 5, 7 e 9. Estas fórmulas são A-estáveis, na classe de métodos para valores de contorno. 
Nome do Solver:

MEBDFI

Autores:

Abdulla and J.R. Cash

Primeira Versão:

Outubro, 2003

Última Modificação:

Linguagem:

Fevereiro, 2006

Link oficial:

Fortran 97

Tipos de Problemas:

http://www.ma.ic.ac.uk/ jcash/IVPsoftware/itest/mebdfi.f

Breve Descrição: O código MEBDFI é uma extensão do MEBDFIDAE para solução de equações diferenciais implícitas e usa o Modified Extended Backward Differentiation Formulas of Cash, que aumentam a região de estabilidade absoluta para BDFs clássicos. Estes métodos são especialmente elaborados para solução de sistemas stiff. As ordens das fórmulas implementadas variam de 1 à 8 .

$\begin{array}{ll}\text { Nome do Solver: } & \text { PSIDE-1 } \\ \text { Autores: } & \text { J.J.B. de Swart, W.M. Lioen, and W.A. van der Veen } \\ \text { Primeira Versão: } & \text { Novembro, 1997 } \\ \text { Ultima Modificação: } & \text { Novembro, 1998 } \\ \text { Linguagem: } & \text { Fortran } 77 \\ \text { Link oficial: } & \text { http://www.ma.ic.ac.uk/ jcash/IVPsoftware/itest/mebdfi.f } \\ \text { Tipos de Problemas: } & \text { IDEs/DAEs de index menores que 3. }\end{array}$

Breve Descrição: Este código usa o método Radau IIA de quatro estágios.

Nome do Solver:

RADAU

Autores:

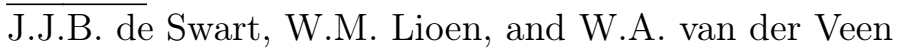

Primeira Versão:

Abril, 1998

Última Modificação:

Linguagem:

Janeiro, 2002

Link oficial:

Fortran 77

http://www.unige.ch/ hairer/prog/stiff/radau.f

Tipos de Problemas: ODES/DAEs de index menores ou igual a 3.

Breve Descrição: O código RADAU é baseado em método em Runge-Kutta Implícitos (Radau

IIa) de ordens 5, 9 e 13. Estes métodos são L-estáveis.

Fixam-se os seguintes termos para as características de simulação dos métodos numéricos.

- Nome do Solver: Podendo ser $\mathrm{MII}_{\text {proj }}$ ou $\mathrm{MII}_{\text {cond }}$, para as novas implementações. BIMD, DDASSL, GAMD, MEBDFI, PSIDE-1 ou RADAU, para os solveres do previamente simulados no [MM08].

- rtol: Tolerância relativa do erro para o integrador numérico.

- atol: Tolerância absoluta do erro para o integrador numérico.

- $h_{0}$ : Tamanho do passo inicial.

- scd: O valor scd denota o número mínimo correto de dígitos significativos na solução numérica no final do intervalo de integração, i.e.:

$$
\text { scd } \doteq-\log _{10}\left(\| \text { erro relativo no final do intervalo de integração } \|_{\infty}\right)
$$

Se algumas das componentes do vetor de solução não são considerados para o computo do valor do scd, ou se o valor absoluto é tomado ao invés do valor relativo, então isto será mencionado no problema em específico. 
- scdY: O valor scdY denota o número mínimo correto de dígitos significativos para a norma do erro da solução em relação à variedade, durante toda a simulação, i.e.:

$$
\operatorname{scd} Y \doteq \max \left\{-\log _{10}\left(\left\|Y^{(\rho)}\right\|_{\infty}\right) ; \forall t \in I\right\}
$$

- steps: Quantidade total de passos dados pelo método (incluindo passos rejeitados por falhas em testes de erro ou em teste de convergência).

- \# $\tau$ e \#QR: quantidade de cálculos do campo $\tau$ e de decomposições QR para $\frac{\partial Y^{\rho-1}}{\partial x}$. (aplicável somente para o MII). Já que no $\mathrm{MII}_{\text {proj }}$ é preciso calcular o complemento ortogonal em cada ponto durante a obtenção da solução, \# $\tau=\# Q R$ para ele.

- \#F, \#Jac e \#LU: quantidade de cálculos da função F, da jacobiana da $\mathrm{F}$ e da decomposição LU para a a Jacobiana de F (aplicável somente para os solveres do TestSet).

- $C P U:$ Tempo de processamento de máquina em segundos para a simulação.

Os problemas simulados são:

\begin{tabular}{|c|c|c|c|}
\hline Abreviatura & Nome do Problema & Área & Referência \\
\hline \hline MatrizCond & Matrizes Mal Condicionadas & exemplo teórico & [SDS05] \\
Pêndulo & Pêndulo Simples sem Amortecimento & mecânica & {$[$ FN02] } \\
SpaceShuttle & Controle de Trajetória Prescrita & mecânica & [AP98] \\
Chemakzo & Chemical Akzo Nobel problem & cinética química & {$[$ [MM08, Sto98] } \\
Andrews & Andrews' squeezing mechanism & mecânica & [MM08, HW96] \\
TransAmp & Amplificador de Transistor & circuitos elétricos & {$[$ [MM08, HLR89] } \\
CarAxis & Eixo de carro & mecânica & [MM08, Sch94] \\
\hline
\end{tabular}

Tabela 4.1: Problemas Simulados: abreviatura, nome, área e referência

Outras informações importantes:

- A menos de menção contrária, é utilizado o método de integração numérica ode $45^{2}$ do $M a t l a b$. Para algumas simulações, utiliza-se também o ode $113^{3}$.

- Os experimentos numéricos foram efetuados num computador com processador Intel Pentium (R) Dual Cpu T3200 2.0GHz, com 2.0Gb de memória RAM.

- Para as simulações, foi utilizado também o parâmetro de convergência $\gamma=0$. Esta escolha foi tomada afim de avaliar o comportamento das soluções sem a presença da componente no campo vetorial corrigindo o erro em relação à variedade $\Gamma$.

\footnotetext{
${ }^{2}$ Da referência de ajuda do Matlab: o ode 45 é um método baseado na associação de métodos de Runge-Kutta de ordens 4 e 5, com controle automático de passo, conhecido como Dormand-Price. Ele é um método de passo único, i.e., para o computo de $y\left(t_{n}\right)$, é necessário somente o conhecimento imediato da solução no ponto anterior $y\left(t_{n-1}\right)$. Em geral, o ode 45 costuma ser uma boa escolha para a primeira tentativa na maioria dos problemas.

${ }^{3}$ Da referência de ajuda do Matlab: o ode113 é um método com controle automático de passo, consistindo num Adamns-Bashforth-Moulton PECE solver de ordens variadas. Ele costuma ser mais eficiente que o ode 45 para tolerâncias rigorosas e quando o avaliação do campo vetorial da EDO requer custo computacional alto. O ode113 é um método de passo múltiplo, ou seja, excetuando-se os primeiros pontos, ele depende das soluções em vários instantes de tempo precedentes para calcular a próxima solução.
} 


\subsection{Problema MatrizCond}

Considere inicialmente o sistema quadrado linear afim invariante no tempo:

$$
\left\{\begin{array}{l}
\dot{x}=\tilde{A} x+\tilde{B} u \\
y=\tilde{C} x \equiv 0
\end{array}\right.
$$

Onde:

$$
\tilde{A}=\left(\begin{array}{rrrr}
0 & -100 & -100 & -100 \\
0 & 0 & 1 & 0 \\
0 & 0 & 0 & 1 \\
0 & 0 & 0 & 0
\end{array}\right), \quad \tilde{B}=\left(\begin{array}{l}
0 \\
0 \\
0 \\
1
\end{array}\right), \quad \tilde{C}=\left(\begin{array}{llll}
0 & 1 & 0 & 0
\end{array}\right)
$$

Com condição inicial $x(0)=x_{0} \doteq(2,0,0,0)$ e $u(0)=0$, para $t \in[0,1000]$.

Assim, escrevendo separadamente as equações do sistema (4.2), obtém-se:

$$
\left\{\begin{aligned}
\dot{x}_{1} & =-100 x_{2}-100 x_{3}-100 x_{4} \\
\dot{x}_{2} & =x_{3} \\
\dot{x}_{3} & =x_{4} \\
\dot{x}_{4} & =u \\
y & =x_{2} \equiv 0
\end{aligned}\right.
$$

De onde verifica-se por substituição que a expressão analítica para a solução de (4.2) é:

$$
x(t)=(2,0,0,0), u(t)=0, \forall t \in[0,1000]
$$

Já que $h(x)=x_{2}$, aplicando-se o processo de derivações de saída sucessivas ao sistema (4.2), conforme Observação 2.6 e Algoritmo 3.2, obtém-se:

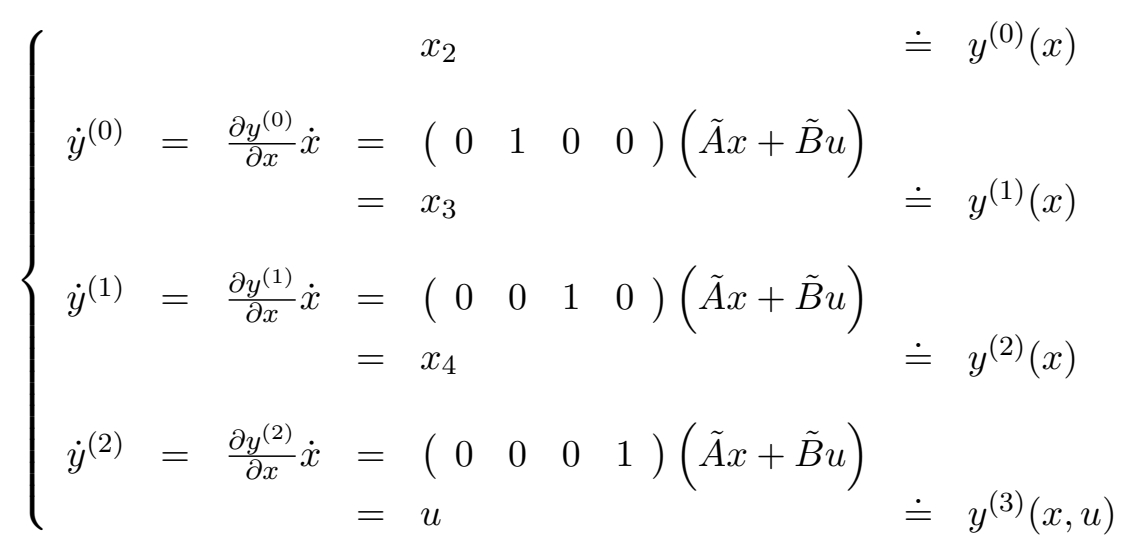

Ou seja, $\rho=3, Y^{(\rho-1)}(x)=\left(x_{2}, x_{3}, x_{4}\right)$ e $y^{(\rho)}(x, u)=u$.

Tomem-se agora uma matriz $K_{4 \times 4}$, constante não singular, e a transformação de coordenadas $w=K^{-1} x$. Assim, o sistema (4.2) escrito nessas novas coordenadas é da forma:

$$
\left\{\begin{array}{l}
\dot{w}=A w+B u \\
y=C w \equiv 0
\end{array}\right.
$$

Onde:

$$
A=K^{-1} \tilde{A} K, \quad B=K^{-1} \tilde{B}, \quad C=\tilde{C} K
$$

Com condição inicial $w(0)=K^{-1} x_{0}$ e $u(0)=0$, para $t \in[0,1000]$. 
Portanto a solução do sistema (4.7) será:

$$
w(t)=K^{-1} x(t)=K^{-1}(2,0,0,0), u(t)=0, \forall t \in[0,1000]
$$

Toma-se então $\left(x_{\text {ref }}, u_{\text {ref }}\right)=\left(x\left(t_{f}\right), u\left(t_{f}\right)\right)\left(K^{-1}(2,0,0,0), 0\right)$. Onde a inversão da matriz foi efetuada simbolicamente.

Daí, como (4.7) foi obtido por uma transformação linear de (4.2). Aplicando-se o processo de derivações de saída sucessivas para o sistema (4.7), também chega-se à $\rho=3, Y^{(\rho-1)}(w)=\left(w_{2}, w_{3}, w_{4}\right)$ e $y^{(\rho)}(x, u)=u$.

Com o intuito de avaliar o efeito de um mal condicionamento, adotou-se a seguinte matriz $\mathrm{K}$ para as simulações:

$$
K=\left(\begin{array}{cccc}
1 & 2 & 3 & 4 \\
4 & 5 & 6 & 7 \\
7 & 8 & 9+10^{-5} & 10 \\
11 & 12 & 13 & 14+10^{-5}
\end{array}\right)
$$

Cujo número de condicionamento é da ordem de $10^{7}$. Assim, o MII será da forma:

$$
\begin{gathered}
\left(\begin{array}{c|c}
\frac{\partial \hat{x}}{\partial w} & 0 \\
\frac{\partial Y^{(\rho-1)}}{\partial w} & 0 \\
\hline \frac{\partial y^{(\rho)}}{\partial w} & \frac{\partial y^{(\rho)}}{\partial u}
\end{array}\right)\left(\begin{array}{c}
\dot{w} \\
\dot{u}
\end{array}\right)=\left(\begin{array}{c}
\left.\frac{\partial \hat{w}}{\partial w}(A w+B u)\right) \\
-\gamma Y^{(\rho)}(w, u)
\end{array}\right) \\
\left(\begin{array}{cccc|c}
\frac{\partial \hat{x}}{\partial w_{1}} & \frac{\partial \hat{x}}{\partial w_{2}} & \frac{\partial \hat{x}}{\partial w_{3}} & \frac{\partial \hat{x}}{\partial w_{4}} & 0 \\
0 & 1 & 0 & 0 & 0 \\
0 & 0 & 1 & 0 & 0 \\
0 & 0 & 0 & 1 & 0 \\
\hline 0 & 0 & 0 & 0 & 1
\end{array}\right)\left(\begin{array}{c}
\dot{w} \\
\dot{u}
\end{array}\right)=\left(\begin{array}{c}
\left.\frac{\partial \hat{x}}{\partial w}(A w+B u)\right) \\
-\gamma w_{2} \\
-\gamma w_{3} \\
-\gamma w_{4}
\end{array}\right)
\end{gathered}
$$

Os resultados das simulações são expostos na tabela abaixo.

\begin{tabular}{|lr|r|rrrrrr|}
\hline Solver & tol $_{\text {cond }}$ & \multicolumn{1}{|c|}{$\gamma$} & scd & scdY & $\# \tau$ & steps & \#QR & CPU \\
\hline \hline MII $_{\text {proj }}$ & & 0 & 2.83 & 7.80 & 407 & 59 & 407 & 0.029 \\
& & 0.1 & 3.87 & 6.60 & 407 & 59 & 407 & 0.029 \\
& & 1 & 4.42 & 2.05 & 2228 & 308 & 2228 & 0.045 \\
& & 10 & 3.86 & 2.39 & 22212 & 3018 & 22212 & 0.228 \\
\hline MII $_{\text {cond }}$ & 10 & 0 & 2.91 & 7.84 & 407 & 59 & 1 & 0.031 \\
& & 0.1 & 3.78 & 6.63 & 407 & 59 & 1 & 0.031 \\
& & 1 & 4.43 & 3.06 & 2239 & 307 & 1 & 0.031 \\
& & 10 & 4.29 & 1.21 & 22247 & 3017 & 1 & 0.130 \\
\hline
\end{tabular}

Tabela 4.2: Problema MatrizCond: $M I I$, rtol $=$ atol $=h_{0}=10^{-4}$

Além disso, o condicionamento dos blocos diagonais de $\mathrm{T}(\mathrm{x}, \mathrm{u})$ foi constante para todos os casos.

\begin{tabular}{|c|cc|}
\hline Solver & cond $([\mathrm{R} ; \mathrm{Yx}])$ & cond $([\mathrm{ypu}]$ \\
\hline \hline $\mathrm{MII}_{\mathrm{proj}}$ & $10^{6}$ & 1 \\
$\mathrm{MII}_{\text {cond }}$ & 1 & 1 \\
\hline
\end{tabular}

Tabela 4.3: Problema MatrizCond: MII, condicionamento 
Análise do Experimento Numérico:

- Para ambos os casos, a precisão da solução $(s c d)$ melhorou com o aumento de $\gamma$, exceto para $\gamma=10$. O maior $(s c d)$ foi atingido para $\gamma=1$.

- O erro em relação à restrição $(s c d Y)$ piorou com o aumento de $\gamma$ em ambos os casos.

- Somente 1 balanceamento foi necessário para o MII $_{\text {cond }}$, pois o problema possui matriz $T(x, u)$ constante.

- Para cada $\gamma$, as características de tempo de processamento (CPU), \#tau e \#steps foram similares nos dois métodos. 


\subsection{Problema Pêndulo}

Considere-se o problema do pêndulo simples sem amortecimento, onde $m$ é uma massa fixada na extremidade inferior de um fio de comprimento $L, g$ é a aceleração da gravidade, $\lambda$ é a tensão no fio e $\theta$ é a coordenada angular contada a partir da vertical no sentido anti-horário. Escolhendo $m=1$ e $L=1$, chega-se a:

$$
\left\{\begin{array}{l}
\ddot{x}=-\lambda x \\
\ddot{y}=-\lambda y-g \\
0 \equiv x^{2}+y^{2}-1^{2}
\end{array}\right.
$$

Com condição inicial $x(0)=x_{0} \doteq(-1,0,0,0)$ e $u(0)=0$, para $t \in\left[0, t_{f}\right]$.

Que, com auxílio das variáveis auxiliares $x_{1} \doteq x, x_{2} \doteq \dot{x}, x_{3} \doteq y, x_{4} \doteq \dot{y}, u \doteq \lambda$, assume a estrutura semi-implícita:

$$
\left\{\begin{aligned}
\dot{x}_{1} & =x_{2} \\
\dot{x}_{2} & =-u x_{1} \\
\dot{x}_{3} & =x_{4} \\
\dot{x}_{4} & =-u x_{3}-g \\
y & =x_{1}^{2}+x_{3}^{2}-1 \equiv 0
\end{aligned}\right.
$$

E já que $h(x)=x_{1}^{2}+x_{3}^{2}-1$, aplicando-se o processo de derivações de saída sucessivas ao sistema (4.13), conforme Observação 2.6 e Algoritmo 3.2, obtém-se:

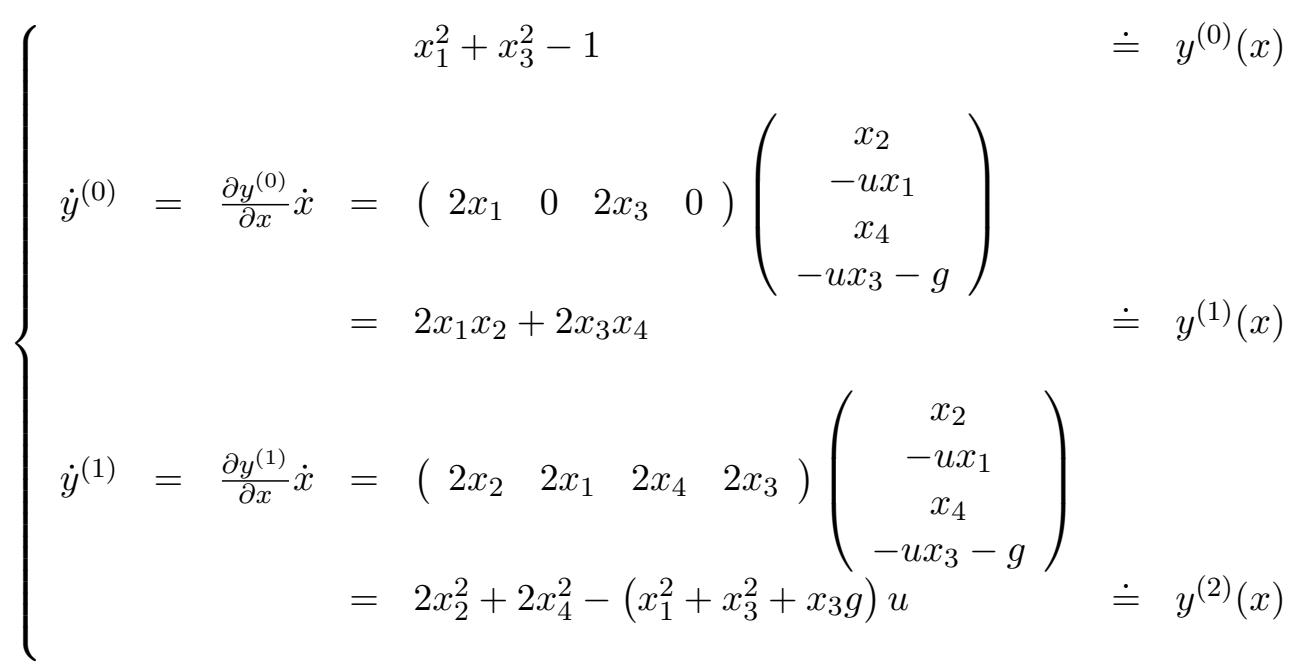

Ou seja, $\rho=2, Y^{(\rho-1)}(x)=\left(x_{1}^{2}+x_{3}^{2}-1,2 x_{1} x_{2}+2 x_{3} x_{4}\right)$ e $y^{(\rho)}(x, u)=2 x_{2}^{2}+2 x_{4}^{2}-\left(x_{1}^{2}+x_{3}^{2}+x_{3} g\right) u$.

Assim, o MII será da forma:

$$
\left(\begin{array}{c|c}
\frac{\partial \hat{x}}{\partial x} & 0 \\
\frac{\partial Y^{(\rho-1)}}{\partial x} & 0 \\
\hline \frac{\partial y^{(\rho)}}{\partial x} & \frac{\partial y^{(\rho)}}{\partial u}
\end{array}\right)\left(\begin{array}{c}
\dot{x} \\
\dot{u}
\end{array}\right)=\left(\begin{array}{c}
\frac{\partial \hat{x}}{\partial x} f(x, u) \\
-\gamma Y^{(\rho)}(x, u)
\end{array}\right)
$$

O instante final da simulação $t_{f}$ será tomado como o período de oscilação do pêndulo, para que $\left(x_{\text {ref }}, u_{\text {ref }}\right) \doteq\left(x\left(t_{f}\right), u\left(t_{f}\right)\right)=(x(0), u(0))$. Esse período é dado pela expressão [FN02]:

$$
t_{f}=4 \sqrt{\frac{l}{g}} \int_{0}^{\pi / 2}\left(\sqrt{1-\left|\sin \frac{\theta_{0}}{2}\right|}\right)^{-1} \sin ^{2} \theta \mathrm{d} \theta
$$

O cálculo dessa integral elíptica é feito com 14 casas decimais de precisão. 
Os resultados das simulações são expostos nas tabelas abaixo.

\begin{tabular}{|lr|r|rrrrrr|}
\hline Solver & tol $_{\text {cond }}$ & \multicolumn{1}{|c|}{$\gamma$} & scd & scdY & $\# \tau$ & steps & $\# \mathrm{QR}$ & $\mathrm{CPU}$ \\
\hline \hline MII $_{\text {proj }}$ & & 0 & 4.30 & 0.93 & 379 & 55 & 379 & 0.029 \\
& & 0.1 & 4.35 & 0.93 & 379 & 55 & 379 & 0.031 \\
& & 1 & 4.58 & 0.92 & 379 & 55 & 379 & 0.030 \\
& & 10 & 3.75 & 0.49 & 379 & 55 & 379 & 0.027 \\
& & 100 & 4.65 & 0.85 & 1114 & 160 & 1114 & 0.031 \\
\hline MII $_{\text {cond }}$ & 10 & 0 & 4.91 & 1.08 & 715 & 103 & 4 & 0.117 \\
& & 0.1 & 4.91 & 1.08 & 715 & 103 & 4 & 0.106 \\
& & 1 & 4.77 & 1.07 & 715 & 103 & 4 & 0.110 \\
& & 10 & 4.03 & 0.65 & 721 & 103 & 4 & 0.110 \\
& & 100 & 4.13 & 0.85 & 1219 & 175 & 4 & 0.095 \\
\hline MII $_{\text {cond }}$ & 5 & 0 & 4.80 & 1.02 & 715 & 103 & 4 & 0.151 \\
& & 0.1 & 4.81 & 1.02 & 715 & 103 & 4 & 0.148 \\
& & 1 & 4.72 & 1.01 & 715 & 103 & 4 & 0.139 \\
& & 10 & 3.91 & 0.59 & 721 & 103 & 4 & 0.140 \\
& & 100 & 4.26 & 0.85 & 1212 & 174 & 4 & 0.141 \\
\hline MII $_{\text {cond }}$ & 2 & 0 & 4.96 & 1.18 & 1905 & 273 & 15 & 0.654 \\
& & 0.1 & 5.00 & 1.17 & 1905 & 273 & 15 & 0.484 \\
& & 1 & 5.12 & 1.11 & 1905 & 273 & 15 & 0.617 \\
& & 10 & 4.33 & 0.68 & 1905 & 273 & 15 & 0.498 \\
& & 100 & 4.55 & 0.85 & 1884 & 270 & 12 & 0.399 \\
\hline
\end{tabular}

Tabela 4.4: Problema Pêndulo: $M I I$, rtol $=$ atol $=h_{0}=10^{-4}$

O condicionamento do bloco ypu na diagonal de $T(x, u)$ foi constantemente igual a 1 em todos os casos. Para o $\mathrm{MII}_{\text {proj }}$, o condicionamento máximo foi inferior à 9.5.

Análise do Experimento Numérico:

- Não há uma tendência clara entre o aumento de $\gamma$ e $s c d$. Considerando-se $s c d, \gamma=1$ está entre as melhores resultados em todos os casos.

- O erro em relação à restrição $s c d Y$ piorou com o aumento de $\gamma$ em ambos os solveres, exceto para $\gamma=100$.

- O custo computacional aumenta com o aumento de $\gamma$ para todos os casos. Em especial para $\gamma=100$ o aumento foi acentuado, indicando uma dinâmica muita rápida em relação ao tamanho do passo de integração, o que dificulta a integração numérica.

- Para o $M I I_{\text {cond }}$ com $t o l_{\text {cond }}=10$ e $t o l_{\text {cond }}=5$ foram necessárias 4 decomposições QR. Para o $M I I_{\text {cond }}$ com $t o l_{\text {cond }}=2$ foi preciso ao menos 3 vezes mais.

- O maior scd foi obtido com $M I I_{\text {cond }}$ com $t o l_{\text {cond }}=2$.

- Considerando conjuntamente o custo computacional (envolvendo $C P U$ e \# $\tau$ ) e precisão $s c d$, $M I I_{\text {proj }} \operatorname{com} \gamma=1$ é a melhor escolha. 


\begin{tabular}{|lr|r|rrrrrr|}
\hline Solver & tol $_{\text {cond }}$ & \multicolumn{1}{|c|}{$\gamma$} & scd & scdY & $\# \tau$ & steps & $\# \mathrm{QR}$ & $\mathrm{CPU}$ \\
\hline \hline MII $_{\text {proj }}$ & & 0 & 5.29 & 1.25 & 505 & 73 & 505 & 0.039 \\
& & 0.1 & 5.33 & 1.23 & 505 & 73 & 505 & 0.025 \\
& & 1 & 5.49 & 1.08 & 545 & 77 & 545 & 0.030 \\
& & 10 & 5.55 & 1.15 & 736 & 106 & 736 & 0.050 \\
& & 100 & 6.88 & 2.11 & 2584 & 370 & 2584 & 0.039 \\
\hline MII $_{\text {cond }}$ & 10 & 0 & 5.59 & 1.26 & 867 & 123 & 4 & 0.109 \\
& & 0.1 & 5.55 & 1.25 & 865 & 123 & 4 & 0.095 \\
& & 1 & 5.37 & 1.17 & 873 & 124 & 4 & 0.280 \\
& & 10 & 5.55 & 1.18 & 986 & 141 & 4 & 0.102 \\
& & 100 & 6.29 & 2.11 & 2780 & 398 & 4 & 0.102 \\
\hline MII $_{\text {cond }}$ & 5 & 0 & 5.59 & 1.25 & 853 & 121 & 4 & 0.100 \\
& & 0.1 & 5.57 & 1.22 & 853 & 121 & 4 & 0.113 \\
& & 1 & 5.45 & 1.16 & 846 & 120 & 4 & 0.135 \\
& & 10 & 5.59 & 1.17 & 959 & 137 & 4 & 0.106 \\
& 100 & 6.36 & 2.13 & 2766 & 396 & 4 & 0.110 \\
\hline MII $_{\text {cond }}$ & 2 & 0 & 5.44 & 1.26 & 2202 & 315 & 15 & 0.422 \\
& & 0.1 & 5.48 & 1.24 & 1881 & 269 & 14 & 0.394 \\
& & 1 & 5.59 & 1.16 & 1895 & 271 & 14 & 0.397 \\
& & 10 & 5.65 & 1.16 & 1960 & 281 & 14 & 0.556 \\
& 100 & 6.66 & 2.11 & 3410 & 488 & 12 & 0.341 \\
\hline
\end{tabular}

Tabela 4.5: Problema Pêndulo: $M I I$, rtol $=$ atol $=h_{0}=10^{-6}$

$\underline{\text { Análise do Experimento Numérico: }}$

- De maneira geral, o aumento de $\gamma$ melhora o $s c d$.

- O erro em relação à restrição $s c d Y$ atingiu menor valor para $\gamma=1$ em todos os casos.

- O custo computacional aumenta com o aumento de $\gamma$ para todos os casos. Em especial para $\gamma=100$ o aumento foi acentuado, indicando uma dinâmica muita rápida em relação ao tamanho do passo de integração, o que dificulta a integração numérica.

- Para o $M I I_{\text {cond }}$ com $t o l_{\text {cond }}=10$ e $t o l_{\text {cond }}=5$ foram necessárias 4 decomposições QR. Para o $M I I_{\text {cond }}$ com $t o l_{\text {cond }}=2$ foi preciso ao menos 3 vezes mais.

- O maior scd foi obtido com $M I I_{\text {cond }}$ com $t o l_{\text {cond }}=2$.

- Considerando conjuntamente o custo computacional (envolvendo $C P U$ e \# $\tau$ ) e precisão $s c d$, $M I I_{\text {proj }} \operatorname{com} \gamma=1$ é a melhor escolha.

- O custo computacional do $M I I_{\text {proj }} \operatorname{com} \gamma=1$ e rtol $=$ atol $=h_{0}=10^{-6}$ foi mais baixo e do que $M I I_{\text {cond }} \operatorname{com} \gamma=1, t_{\text {cond }}=2 \mathrm{e}$ rtol $=$ atol $=h_{0}=10^{-4}$, contudo a precisão $s c d$ foi maior. Nesse caso o refinamento do passo de integração para o $M I I_{\text {proj }}$ foi mais vantajoso que a imposição de uma tolerância menor no condicionamento para o $M I I_{\text {cond }}$. 


\subsection{Problema SpaceShuttle}

Na simulação de veículos espaciais, o formato da trajetória é frequentemente imposto adicionandose um conjunto de restrições às equações de movimento.

O modelo de equações então torna-se um sistema de DAEs não linear semi-implícito. As equações diferenciais incluem as equações clássicas de movimento, e as equações algébricas correspondem às restrições de percurso. Dá-se o nome de Trajectory prescribed path control - TPPC para essa classe de problemas.

O problema tratado aqui é um TPPC de 6 equações diferenciais, 2 equações algébricas e index 2, presente em [AP98] Seção 6.3, da forma:

$$
\left\{\begin{array}{l}
\dot{x}=f(t, x, u) \\
y=h(t, x) \equiv 0
\end{array}\right.
$$

Com uma condição inicial $(x(0), u(0))=\left(x_{0}, u_{0}\right)$ dada, para $t \in[0,300]$.

Utilizou a solução obtida através do $M I I_{\text {proj }}$ com rtol $=$ atol $=h_{0}=10^{-14}$ como $\left(x_{\text {ref }}, u_{\text {ref }}\right)$, visto que a fonte [AP98] não disponibiliza uma solução suficientemente precisa para comparação em $t=300$. Além disso, provavelmente por problemas de tipográficos em [AP98], algumas constantes na definição do problema estavam incorretas e precisaram ser readequadas para que $\left\|h\left(0, x_{0}\right)\right\|$ fosse suficientemente pequena. As constantes utilizadas bem como a solução de referência encontram-se no apêndice, dentro dos códigos fonte.

Os resultados das simulações são expostos nas tabelas abaixo.

\begin{tabular}{|lr|r|rrrrrr|}
\hline Solver & tol $_{\text {cond }}$ & \multicolumn{1}{|c|}{$\gamma$} & scd & scdY & $\# \tau$ & steps & $\# \mathrm{QR}$ & $\mathrm{CPU}$ \\
\hline \hline MII $_{\text {proj }}$ & & 0 & 4.51 & 2.83 & 400 & 58 & 400 & 0.027 \\
& & 0.1 & 3.60 & 1.92 & 400 & 58 & 400 & 0.027 \\
& & 1 & 2.76 & 1.30 & 843 & 120 & 834 & 0.051 \\
& & 10 & 2.59 & 1.23 & 6430 & 916 & 6430 & 0.050 \\
\hline MII $_{\text {cond }}$ & 10 & 0 & 6.31 & 2.84 & 1246 & 180 & 10 & 0.253 \\
& & 0.1 & 4.86 & 1.93 & 1248 & 180 & 10 & 0.267 \\
& & 1 & 4.85 & 1.39 & 1421 & 204 & 8 & 0.194 \\
& & 10 & 2.96 & 1.23 & 6722 & 956 & 8 & 0.231 \\
\hline MII $_{\text {cond }}$ & 5 & 0 & 6.20 & 2.84 & 2066 & 299 & 22 & 0.602 \\
& & 0.1 & 4.85 & 1.93 & 2072 & 299 & 22 & 0.573 \\
& & 1 & 4.64 & 1.39 & 1882 & 270 & 15 & 0.422 \\
& & 10 & 3.23 & 1.17 & 6924 & 948 & 13 & 0.391 \\
\hline
\end{tabular}

Tabela 4.6: Problema SpaceShuttle: $M I I$, rtol $=$ atol $=h_{0}=10^{-4}$

\begin{tabular}{|cc|c|crrrrr|}
\hline Solver & rtol & $\gamma$ & scd & scdY & $\# \tau$ & steps & \#QR & CPU \\
\hline \hline MII $_{\text {proj }}$ & $10^{-6}$ & 1 & 4.73 & 2.61 & 1709 & 245 & 1709 & 0.100 \\
& $10^{-7}$ & 1 & 5.61 & 3.23 & 2577 & 369 & 2557 & 0.135 \\
\hline
\end{tabular}

Tabela 4.7: Problema SpaceShuttle: $M I_{\text {proj }}, r t o l=$ atol $=h_{0}$, refinamento de passo 
Análise do Experimento Numérico:

- O aumento de $\gamma$ piorou scd e $s c d Y$.

- O custo computacional aumenta com o aumento de $\gamma$ para todos os casos. Em especial para $\gamma=10$ o aumento foi acentuado.

- Para o $M I I_{\text {cond }}$, o aumento de $t o l_{\text {cond }}$ exigiu mais decomposições QR.

- O maior scd foi obtido com $M I I_{\text {cond }}$ com $t o l_{\text {cond }}=5$ e $\gamma=0$.

- Considerando conjuntamente o custo computacional (envolvendo $C P U$ e \# $\pi$ ) e precisão $s c d$, $M I I_{\text {proj }} \operatorname{com} \gamma=1$ não foi a melhor escolha, mas não foi a pior.

- Nesse caso o refinamento do passo de integração para o $M I I_{\text {proj }}$ não foi mais vantajoso que a imposição de uma tolerância menor no condicionamento para o $\overline{M I} I_{\text {cond }}$. 


\subsection{Problema Chemakzo}

Nome do Problema: Chemical Akzo Nobel problem

Contribuição: parallel-IVP-algorithm group of CWI.

Ano: $\quad 1996$

Área: $\quad$ cinética química.

Breve Descrição Este PVI é um sistema de EDAs stiff com 6 equações e index 1. O problema é originário do Akzo Nobel Central research in Arnhern e descreve o processo químico no qual duas espécies químicas são misturadas, enquanto dióxido de carbono é adicionado continuamente.

O problema foi reescrito na forma:

$$
\left\{\begin{array}{l}
\dot{x}=f(x, u) \\
y=h(x, u) \equiv 0
\end{array}\right.
$$

Com $x \in \mathbb{R}^{5}$ e $u \in \mathbb{R}$, condição inicial $(x(0), u(0))=\left(x_{0}, u_{0}\right)$ dada, e $t \in[0,180]$. A solução de referência ao final do intervalo de integração $\left(x_{\text {ref }}, u_{\text {ref }}\right)$ também foi fornecida.

Os resultados das simulações são expostos nas tabelas abaixo.

\begin{tabular}{|lr|r|rrrrrr|}
\hline Solver tol & \multicolumn{1}{|c|}{$\gamma$} & scd & scdY & $\# \tau$ & steps & $\# \mathrm{QR}$ & $\mathrm{CPU}$ \\
\hline \hline MII $_{\text {proj }}$ & & 0 & 10.99 & 5.72 & 3780 & 540 & 3780 & 0.040 \\
& & 0.1 & 10.99 & 5.58 & 3780 & 540 & 3780 & 0.050 \\
& & 1 & 10.91 & 6.45 & 4159 & 595 & 4159 & 0.040 \\
& & 10 & 10.84 & 7.22 & 13805 & 1973 & 13805 & 0.070 \\
& & 100 & 11.15 & 8.00 & 62329 & 8905 & 62329 & 0.192 \\
\hline MII $_{\text {cond }}$ & 10 & 0 & 10.99 & 5.72 & 3780 & 540 & 1 & 0.063 \\
& & 0.1 & 10.99 & 5.58 & 3780 & 540 & 1 & 0.045 \\
& & 1 & 10.91 & 6.45 & 4159 & 595 & 1 & 0.046 \\
& 10 & 10.84 & 7.22 & 13805 & 1973 & 1 & 0.090 \\
& 100 & 11.15 & 8.00 & 62329 & 8905 & 1 & 0.273 \\
\hline
\end{tabular}

Tabela 4.8: Problema Chemakzo: MII, rtol $=$ atol $=h_{0}=10^{-10}$

Nesse problema a saída já depende explicitamente de $\mathrm{u}$, assim $\rho=0$. Além disso, yu $\equiv-1$ e os condicionamento foram constantes para o $\mathrm{MII}_{\text {proj }}: \operatorname{cond}([\mathrm{R} ; \mathrm{Yx}]) \equiv 1$ e $\operatorname{cond}(\mathrm{yu}) \equiv 1$.

Análise do Experimento Numérico:

- A menos das características $C P U$ e \#QR, ambos os solveres exibiram comportamento idêntico, devido ao fato de $\rho$ ser nulo.

- O aumento de $\gamma$ piorou $s c d$, exceto para $\gamma=100$.

- O aumento de $\gamma$ melhorou $s c d Y$ para todos o $\gamma$. 
Os resultados apresentados a seguir foram extraídos de [MM08], utilizando outros solveres implementados em Fortran.

\begin{tabular}{|lccc|rrrrrrr|}
\hline \multicolumn{1}{|c|}{ Solver } & rtol & atol & $h_{0}$ & scd & steps & accept & \#F & \#Jac & \#LU & CPU \\
\hline \hline BIMD & $10^{-10}$ & $10^{-10}$ & $10^{-10}$ & 10.61 & 41 & 41 & 1177 & 41 & 41 & 0.0039 \\
DDASSL & $10^{-10}$ & $10^{-10}$ & & 8.33 & 522 & 515 & 649 & 38 & & 0.0039 \\
GAMD & $10^{-10}$ & $10^{-10}$ & $10^{-10}$ & 9.82 & 35 & 35 & 1737 & 35 & 35 & 0.0039 \\
MEBDFI & $10^{-10}$ & $10^{-10}$ & $10^{-10}$ & 9.76 & 274 & 273 & 916 & 32 & 32 & 0.0029 \\
PSIDE-1 & $10^{-10}$ & $10^{-10}$ & & 9.91 & 87 & 85 & 1671 & 15 & 204 & 0.0039 \\
RADAU & $10^{-10}$ & $10^{-10}$ & $10^{-10}$ & 8.39 & 43 & 41 & 696 & 30 & 43 & 0.0010 \\
\hline
\end{tabular}

Tabela 4.9: Problema Chemakzo: Outros Solveres

Comparação com outros métodos:

- Tome-se como base o resultado obtido para o $M I I_{\text {proj }} \operatorname{com} \gamma=1$.

- Comparando com a tabela acima, o scd foi melhor do que o dos outros solveres.

- A quantidade de passos steps ficou próxima da faixa dos outros solveres.

- A quantidade de cálculo de $\tau$ foi ao menos 4 vezes maior que a quantidade de cálculos de $f$ dos outros solveres. Por outro lado, este custo pode ser reduzido utilizando um método de integração numérica de passo múltiplo, como ilustrado abaixo, mantendo ainda bons resultados.

\begin{tabular}{|rr|r|rrrrrr|}
\hline Método & integrador & $\gamma$ & scd & scdY & $\# \tau$ & steps & \#QR & CPU \\
\hline \hline MII $_{\text {proj }}$ & ode113 & 1 & 10.29 & 8.94 & 2072 & 681 & 2072 & 0.060 \\
\hline
\end{tabular}

Tabela 4.10: Problema Chemakzo: $M I I_{\text {proj }}$, rtol $=$ atol $=h_{0}=10^{-10}$, ode113 


\subsection{Problema Andrews}

Nome do Problema: Andrews'squeezing mechanism problem

Contribuição: parallel-IVP-algorithm group of CWI

Ano: $\quad 1996$

Área: $\quad$ mecânica

Breve Descrição: Este PVI é um sistema EDAs não-stiff de segunda ordem e index 3, constituído de 14 equações diferenciais e 13 equações algébricas. O problema descreve o movimento de 7 corpos rígidos conectado por articulações sem friç̧ão.

O problema foi reescrito na forma:

$$
\left\{\begin{array}{l}
\dot{x}=f(x, u) \\
y=h(x) \equiv 0
\end{array}\right.
$$

Com $x \in \mathbb{R}^{14}$ e $u \in \mathbb{R}^{13}$, condição inicial $(x(0), u(0))=\left(x_{0}, u_{0}\right)$ dada, e $t \in[0,0.03]$. A solução de referência ao final do intervalo de integração $\left(x_{\text {ref }}, u_{\text {ref }}\right)$ também foi fornecida, observando-se que somente as 7 primeiras coordenadas da solução de referência foram utilizadas para o cálculo de $s c d$, tal como feito por [MM08].

Os resultados das simulações são expostos nas tabelas abaixo.

\begin{tabular}{|lr|r|rrrrrr|}
\hline Solver & tol $_{\text {cond }}$ & \multicolumn{1}{|c|}{$\gamma$} & scd & scdY & $\# \tau$ & steps & $\# \mathrm{QR}$ & \multicolumn{1}{c|}{ CPU } \\
\hline \hline MII $_{\text {proj }}$ & & 0 & 2.02 & -2.46 & 581 & 77 & 581 & 0.052 \\
& & 0.1 & 2.02 & -2.46 & 581 & 77 & 581 & 0.042 \\
& & 1 & 2.02 & -2.57 & 581 & 77 & 581 & 0.051 \\
& & 10 & 2.05 & -2.56 & 600 & 78 & 600 & 0.047 \\
& & 100 & 2.41 & -2.50 & 613 & 79 & 613 & 0.048 \\
\hline MII $_{\text {cond }}$ & 1000 & 1 & 5.29 & 1.19 & 38082 & 21561 & 4293 & 57.000 \\
\hline
\end{tabular}

Tabela 4.11: Problema Andrews: $M I I$, rtol $=$ atol $=h_{0}=10^{-4}$

\begin{tabular}{|cc|r|rrrrrr|}
\hline Solver & tol $_{\text {cond }}$ & \multicolumn{1}{|c|}{$\gamma$} & scd & scdY & \multicolumn{1}{c}{$\# \tau$} & steps & \#QR & \multicolumn{1}{c|}{ CPU } \\
\hline \hline MII $_{\text {proj }}$ & & 0 & 5.75 & -0.33 & 2315 & 323 & 2315 & 0.062 \\
& & 0.1 & 5.75 & -0.33 & 2290 & 322 & 2290 & 0.060 \\
& & 1 & 5.76 & -0.33 & 2284 & 322 & 2284 & 0.059 \\
\hline MII $_{\text {cond }}$ & 1000 & 1 & 8.20 & 1.28 & 178036 & 103839 & 8287 & 191.000 \\
\hline
\end{tabular}

Tabela 4.12: Problema Andrews: $M I I$, rtol $=$ atol $=h_{0}=10^{-7}$

\begin{tabular}{|cc|c|crrrrr|}
\hline Solver & rtol & $\gamma$ & scd & scdY & $\# \tau$ & steps & $\# \mathrm{QR}$ & $\mathrm{CPU}$ \\
\hline \hline MII $_{\text {proj }}$ & $10^{-9}$ & 1 & 8.21 & 0.51 & 5865 & 837 & 5865 & 0.088 \\
& $10^{-11}$ & 1 & 8.86 & 1.32 & 14980 & 2140 & 14980 & 0.143 \\
\hline
\end{tabular}

Tabela 4.13: Problema Andrews: $M I_{\text {proj }}$, rtol $=$ atol $=h_{0}$, refinamento de passo 
Análise do Experimento Numérico:

- O aumento de $\gamma$ melhorou scd.

- Para rtol $=10^{-4}$ o aumento de $\gamma$ melhorou $s c d Y$, exceto para $\gamma=100$. Para rtol $=10^{-7} \mathrm{o}$ aumento de $\gamma$ não modificou $s c d Y$, exceto para $\gamma=100$.

- Não há uma relação clara entre o aumento de $\gamma$ e um maior custo computacional ( $C P U$ e steps).

- Para o $M I I_{\text {cond }}$, tolerâncias de condicionamento mais baixas impossibilitaram na prática a simulação, devido ao tempo de processamento requerido. Mostram-se nas tabelas anteriores o resultado somente para $t o l_{\text {cond }}=1000$. De fato, obtiveram-se 3 dígitos a mais de precisão no $s c d$, contudo a quantidade de cálculos de $\tau$ e o tempo de processamento $C P U$ foram ao menos 70 vezes maiores.

- Considerando conjuntamente o custo computacional (envolvendo $C P U$ e \# $\tau$ ) e precisão scd, $M I I_{\text {proj }} \operatorname{com} \gamma=1$ foi uma boa escolha.

- Nesse caso o refinamento do passo de integração para o $M I I_{\text {proj }}$ foi mais vantajoso que o uso do $M I I_{\text {cond }}$.

\begin{tabular}{|cccc|crrrrrr|}
\hline Solver & rtol & atol & $h_{0}$ & scd & steps & accept & \#F & \#Jac & \#LU & CPU \\
\hline \hline BIMD & $10^{-4}$ & $10^{-4}$ & $10^{-4}$ & 3.05 & 46 & 41 & 1034 & 41 & 46 & 0.0185 \\
& $10^{-7}$ & $10^{-7}$ & $10^{-7}$ & 5.38 & 122 & 122 & 2553 & 122 & 122 & 0.0459 \\
GAMD & $10^{-4}$ & $10^{-4}$ & $10^{-4}$ & 2.82 & 82 & 58 & 2281 & 58 & 82 & 0.0293 \\
& $10^{-7}$ & $10^{-7}$ & $10^{-7}$ & 4.54 & 128 & 116 & 5176 & 116 & 128 & 0.0693 \\
MEBDFI & $10^{-4}$ & $10^{-4}$ & $10^{-4}$ & 0.37 & 118 & 108 & 466 & 23 & 23 & 0.0078 \\
& $10^{-7}$ & $10^{-7}$ & $10^{-7}$ & 3.50 & 300 & 287 & 1222 & 38 & 38 & 0.0195 \\
PSIDE-1 & $10^{-4}$ & $10^{-4}$ & & 2.95 & 92 & 75 & 1675 & 52 & 368 & 0.0410 \\
& $10^{-7}$ & $10^{-7}$ & & 4.98 & 113 & 93 & 2637 & 63 & 428 & 0.0615 \\
RADAU & $10^{-4}$ & $10^{-4}$ & $10^{-4}$ & 1.36 & 96 & 56 & 810 & 54 & 96 & 0.0137 \\
& $10^{-7}$ & $10^{-7}$ & $10^{-7}$ & 4.45 & 114 & 95 & 1292 & 90 & 114 & 0.0195 \\
\hline
\end{tabular}

Tabela 4.14: Problema Andrews: Outros Solveres

Comparação com outros métodos:

- Tome-se como base o resultado obtido para o $M I I_{\text {proj }} \operatorname{com} \gamma=1$.

- Comparando com a tabela acima, o scd foi melhor do que o dos outros solveres.

- A quantidade de passos steps foi ao menos duas vezes maior que a dos outros solveres.

- A quantidade de cálculos de $\tau$ ficou próxima da quantidade mais baixa dentre os outros solveres. 


\subsection{Problema TransAmp}

Nome do Problema: Amplificador de Transistor

Contribuição: $\quad$ parallel-IVP-algorithm group of CWI.

Ano: 1996

Área: análise de circuitos elétricos

Breve Descrição: Este PVI é um sistema EDAs stiff de index 1, constituído de 8 equações. O problema origina-se da análise de circuitos elétricos, sendo um modelo de amplificador de transistor.

Uma particularidade deste problema é que ele foi dado na forma:

$$
\left\{M \frac{d}{d t}(w)=F(t, w)\right.
$$

Com $w(0)=w_{0}, w^{\prime}(0)=w_{0}^{\prime}, w \in \mathbb{R}^{N}$ e $t \in[0,0.2]$. Observando-se que $M$ é uma matriz constante singular, com a qual não é imediata, como nos benchmarks anteriores, a reformulação necessária.

Então, para transformá-lo num problema semi-implícito, será utilizada a decomposição em valores singulares, conforme o Lema 3.12, obtendo $U S V^{\top}=M \operatorname{com} S$ de posto $n$, da seguinte maneira:

$$
\begin{aligned}
M \dot{w} & =F(t, w) \\
\Leftrightarrow \quad U S V^{\top} \dot{w} & =F(t, w) \\
\Leftrightarrow S\left(\begin{array}{c}
\dot{x} \\
\dot{u}
\end{array}\right) & =\left(\begin{array}{c}
F_{1}(t, x, u) \\
F_{2}(t, x, u)
\end{array}\right)
\end{aligned}
$$

Onde $\left(\begin{array}{l}x \\ u\end{array}\right) \doteq V^{\top} w, \tilde{F}(t, x, u)=U^{\top} F\left(t, V\left(\begin{array}{l}x \\ u\end{array}\right)\right)$ e $\left(\tilde{F}_{1}, \tilde{F}_{2}\right) \doteq \tilde{F}$, onde $\tilde{F}_{1}$ representa as $n$ primeiras componentes de $\tilde{F}$ e $\tilde{F}_{2}$ representa as últimas $m \doteq N-n$ componentes de $\tilde{F}$.

Como $S=\left(\begin{array}{c|c}s_{n \times n} & 0 \\ \hline 0 & 0\end{array}\right)$, já que $S$ é uma matriz diagonal não negativa, ordenada de maneira decrescente, pode-se escrever:

$$
\left\{\begin{array}{l}
\dot{x}=f(t, x, u) \\
y=h(t, x, u) \equiv 0
\end{array}\right.
$$

Onde $f(t, x, u) \doteq s^{-1} \tilde{F}_{1}(t, x, u)$ e $h(t, x, u) \doteq \tilde{F}_{2}(t, x, u)$. Com condição inicial $(x(0), u(0))=V^{\top} w_{0}$, para o mesmo intervalo de tempo $t \in[0,0.2]$

\begin{tabular}{|lc|r|rrrrrr|}
\hline Solver & tol $_{\text {cond }}$ & \multicolumn{1}{|c|}{$\gamma$} & scd & scdY & $\# \tau$ & steps & $\# \mathrm{QR}$ & $\mathrm{CPU}$ \\
\hline \hline MII $_{\text {proj }}$ & & 0 & 1.25 & 1.11 & 10567 & 1429 & 10567 & 0.097 \\
& & 0.1 & 1.34 & 1.11 & 10574 & 1430 & 10574 & 0.100 \\
& & 1 & 1.55 & 1.09 & 10588 & 1432 & 10588 & 0.102 \\
& & 10 & 1.67 & 0.91 & 10639 & 1435 & 10639 & 0.114 \\
& & 100 & 2.70 & 1.16 & 10831 & 1465 & 10831 & 0.086 \\
\hline MII $_{\text {cond }}$ & 1000 & 1 & 2.07 & 1.19 & 59422 & 8452 & 1859 & 51.755 \\
\hline
\end{tabular}

Tabela 4.15: Problema Transamp: MII, rtol $=$ atol $=10^{-4}, h_{0}=10^{-6}$ 


\begin{tabular}{|cc|r|rrrrrr|}
\hline Solver & tol $_{\text {cond }}$ & \multicolumn{1}{|c|}{$\gamma$} & scd & scdY & $\# \tau$ & steps & $\# \mathrm{QR}$ & $\mathrm{CPU}$ \\
\hline \hline $\mathrm{MII}_{\text {proj }}$ & & 0 & 5.10 & 2.57 & 38304 & 5443 & 38304 & 0.268 \\
& & 0.1 & 5.11 & 2.58 & 38304 & 5443 & 38304 & 0.223 \\
& & 1 & 5.16 & 2.58 & 38298 & 5443 & 38298 & 0.228 \\
& & 10 & 5.65 & 2.65 & 38395 & 5456 & 38395 & 0.255 \\
& & 100 & 7.17 & 2.66 & 39242 & 5571 & 39242 & 0.238 \\
\hline MII $_{\text {cond }}$ & 1000 & 1 & 3.92 & 2.80 & 90722 & 13058 & 998 & 27.511 \\
\hline
\end{tabular}

Tabela 4.16: Problema Transamp: $M I I$, rtol $=$ atol $=10^{-7}, h_{0}=10^{-9}$

Análise do Experimento Numérico:

- O aumento de $\gamma$ melhorou scd.

- Para rtol $=10^{-} 4$ a relação entre o aumento $\gamma$ e $s c d Y$ não é clara. Para rtol $=10^{-} 7$ o aumento de $\gamma$ melhorou $s c d Y$.

- O aumento de $\gamma$ acarretou um maior custo computacional (CPU e steps).

- Para o $M I I_{\text {cond }}$, tolerâncias de condicionamento mais baixas impossibilitaram na prática a simulação, devido ao tempo de processamento requerido. Mostram-se nas tabelas anteriores o resultado somente para $t o l_{\text {cond }}=1000$. Mesmo assim o ganho em precisão expressivo e o custo computacional foi consideravelmente maior tanto para rtol $=10^{-} 4$ como $10^{-} 7$.

- Considerando conjuntamente o custo computacional (envolvendo $C P U$ e \# $\pi$ ) e precisão $s c d$, $M I I_{\text {proj }} \operatorname{com} \gamma=1$ não foi a melhor escolha, mas ficou entre as melhores.

\begin{tabular}{|cccc|crrrrrr|}
\hline Solver & rtol & atol & $h_{0}$ & scd & steps & accept & \#F & \#Jac & \#LU & CPU \\
\hline \hline BIMD & $10^{-4}$ & $10^{-4}$ & $10^{-6}$ & 5.63 & 466 & 408 & 8423 & 408 & 466 & 0.0322 \\
& $10^{-7}$ & $10^{-7}$ & $10^{-9}$ & 8.34 & 618 & 575 & 19632 & 575 & 618 & 0.0752 \\
DDASSL & $10^{-4}$ & $10^{-4}$ & & 3.08 & 9759 & 6026 & 18381 & 7359 & & 0.1113 \\
& $10^{-7}$ & $10^{-7}$ & & 5.49 & 40810 & 23859 & 77402 & 33678 & & 0.4743 \\
GAMD & $10^{-4}$ & $10^{-4}$ & $10^{-6}$ & 5.83 & 373 & 276 & 17204 & 276 & 373 & 0.0517 \\
& $10^{-7}$ & $10^{-7}$ & $10^{-9}$ & 7.37 & 374 & 325 & 34320 & 326 & 374 & 0.1064 \\
MEBDFI & $10^{-4}$ & $10^{-4}$ & $10^{-6}$ & 4.80 & 1580 & 1486 & 5949 & 256 & 256 & 0.0303 \\
& $10^{-7}$ & $10^{-7}$ & $10^{-9}$ & 6.99 & 3628 & 3513 & 13324 & 419 & 419 & 0.0703 \\
PSIDE-1 & $10^{-4}$ & $10^{-4}$ & & 4.76 & 516 & 362 & 9742 & 253 & 2008 & 0.0351 \\
& $10^{-7}$ & $10^{-7}$ & & 7.23 & 653 & 653 & 21914 & 419 & 2724 & 0.0732 \\
RADAU & $10^{-7}$ & $10^{-7}$ & $10^{-9}$ & 6.83 & 1551 & 1551 & 17582 & 1541 & 1775 & 0.0517 \\
\hline
\end{tabular}

Tabela 4.17: Problema TransAmp: Outros Solveres

Comparação com outros métodos:

- Tome-se como base o resultado obtido para o $M I I_{\text {proj }} \operatorname{com} \gamma=1$.

- Comparando com a tabela acima, o scd foi pior que todos os outros solveres, indicando que apesar do artifício registrado acima possibilitar a estruturação de $M \dot{w}=F(w)$ como um sistema semi-implícito, esta não é uma boa opção prática comparando-se com outros solveres que lidam diretamente com essa classe de problemas. 


\subsection{Problema CarAxis}

\begin{tabular}{ll} 
Nome do Problema: & Eixo de Carro \\
Contribuição: & S. Schneider \\
\hline Ano: & 1996 \\
Ârea: & mecânica
\end{tabular}

Breve Descrição: Este problema é um sistema EDAs stiff de index 3, constituído 8 equações diferenciais e 2 equações algébricas. Ele é um exemplo simplificado de sistema com múltiplos corpos, no qual o comportamento do eixo de um carro numa via esburacada é modelado como um conjunto de equações algébrico-diferenciais. O problema foi reescrito na forma:

$$
\left\{\begin{array}{l}
\dot{x}=f(t, x, u) \\
y=h(t, x) \equiv 0
\end{array}\right.
$$

Com $x \in \mathbb{R}^{8}$ e $u \in \mathbb{R}^{2}$, condição inicial $(x(0), u(0))=\left(x_{0}, u_{0}\right)$ dada, e $t \in[0,3]$. A solução de referência ao final do intervalo de integração $\left(x_{\text {ref }}, u_{\text {ref }}\right)$ também foi fornecida.

Os resultados das simulações são expostos nas tabelas abaixo.

\begin{tabular}{|lr|r|rrrrrr|}
\hline Solver & tol $_{\text {cond }}$ & \multicolumn{1}{|c|}{$\gamma$} & scd & scdY & steps & $\# \tau$ & $\# \mathrm{QR}$ & $\mathrm{CPU}$ \\
\hline \hline MII $_{\text {proj }}$ & & 0 & 0.99 & -0.17 & 2061 & 261 & 2061 & 0.044 \\
& & 1 & 1.38 & -0.06 & 2030 & 260 & 2030 & 0.044 \\
& & 10 & 2.56 & -0.13 & 1985 & 257 & 1985 & 0.046 \\
& 100 & 1.91 & 0.32 & 2285 & 317 & 2285 & 0.045 \\
\hline MII $_{\text {cond }}$ & 10 & 0 & 1.48 & -0.19 & 3245 & 425 & 20 & 0.574 \\
& & 1 & 2.18 & -0.23 & 2606 & 338 & 15 & 0.451 \\
& & 10 & 2.20 & -0.19 & 2654 & 350 & 19 & 0.548 \\
& & 100 & 3.02 & -2.41 & 2889 & 399 & 21 & 0.611 \\
\hline MII $_{\text {cond }}$ & 5 & 0 & 1.64 & -0.09 & 3823 & 523 & 40 & 1.703 \\
& & 1 & 1.79 & -0.11 & 3783 & 513 & 37 & 1.710 \\
& & 10 & 2.28 & -0.16 & 3773 & 515 & 38 & 1.602 \\
& 100 & 2.61 & 0.02 & 4019 & 563 & 37 & 1.604 \\
\hline
\end{tabular}

Tabela 4.18: Problema CarAxis: MII, rtol $=$ atol $=h_{0}=10^{-4}$

\begin{tabular}{|lr|r|rrrrrr|}
\hline Solver & tol $_{\text {cond }}$ & \multicolumn{1}{|c|}{$\gamma$} & scd & scdY & $\# \tau$ & steps & $\# \mathrm{QR}$ & $\mathrm{CPU}$ \\
\hline \hline $\mathrm{MII}_{\text {proj }}$ & & 0 & 3.85 & 2.26 & 6673 & 949 & 6673 & 0.074 \\
& & 1 & 4.19 & 2.23 & 6673 & 949 & 6673 & 0.072 \\
& & 10 & 4.93 & 2.25 & 6665 & 947 & 6665 & 0.101 \\
& & 100 & 6.25 & 1.72 & 8739 & 1245 & 8739 & 0.103 \\
\hline MII $_{\text {cond }}$ & 10 & 0 & 4.33 & 1.86 & 8759 & 1228 & 29 & 0.845 \\
& & 1 & 4.84 & 2.05 & 8429 & 1185 & 24 & 0.674 \\
& & 10 & 5.56 & 1.97 & 8708 & 1225 & 28 & 0.787 \\
& & 100 & 6.17 & 1.75 & 10508 & 1491 & 28 & 0.945 \\
\hline MII $_{\text {cond }}$ & 5 & 0 & 4.52 & 1.59 & 8733 & 1223 & 30 & 1.284 \\
& & 1 & 4.72 & 1.91 & 8717 & 1224 & 32 & 1.528 \\
& & 10 & 5.39 & 2.03 & 8806 & 1243 & 32 & 1.386 \\
& & 100 & 6.48 & 1.68 & 10678 & 1519 & 36 & 1.571 \\
\hline
\end{tabular}

Tabela 4.19: Problema CarAxis: $M I I$, rtol $=$ atol $=h_{0}=10^{-7}$ 


\begin{tabular}{|lr|r|rrrrrr|}
\hline Solver & tol $_{\text {cond }}$ & \multicolumn{1}{|c|}{$\gamma$} & scd & scdY & $\# \tau$ & steps & \#QR & CPU \\
\hline \hline MII $_{\text {proj }}$ & & 0 & 7.71 & 3.60 & 26475 & 3783 & 26475 & 0.195 \\
& & 1 & 8.09 & 3.60 & 26468 & 3782 & 26468 & 0.242 \\
& & 10 & 8.92 & 3.58 & 26552 & 3794 & 26552 & 0.241 \\
& & 100 & 9.33 & 3.47 & 34497 & 4929 & 34497 & 0.297 \\
\hline MII $_{\text {cond }}$ & 10 & 0 & 7.67 & 3.51 & 28909 & 4148 & 28 & 0.883 \\
& & 1 & 8.05 & 3.43 & 28906 & 4148 & 28 & 0.895 \\
& & 10 & 8.91 & 3.53 & 28798 & 4133 & 28 & 0.866 \\
& & 100 & 9.40 & 3.45 & 36446 & 5226 & 28 & 1.072 \\
\hline MII $_{\text {cond }}$ & 5 & 0 & 7.41 & 3.44 & 29105 & 4180 & 38 & 1.869 \\
& & 1 & 7.80 & 3.44 & 28948 & 4159 & 36 & 2.051 \\
& & 10 & 8.76 & 3.43 & 28802 & 4135 & 34 & 1.764 \\
& & 100 & 9.51 & 3.47 & 36385 & 5223 & 38 & 2.417 \\
\hline
\end{tabular}

Tabela 4.20: Problema CarAxis: $M I I$, rtol $=$ atol $=h_{0}=10^{-10}$

Análise do Experimento Numérico:

- O aumento de $\gamma$ melhorou $s c d$, exceto para rtol $=10^{-} 4$ e $\gamma=100$

- O aumento de $\gamma$ não melhorou necessariamente $s c d Y$.

- De maneira geral o aumento de $\gamma$ acarretou um maior custo computacional (CPU e steps).

- $\mathrm{O} M I I_{\text {cond }}$ aumentou o custo computacional e não tornou a solução mais precisa quanto à scd.

- Considerando conjuntamente o custo computacional (envolvendo $C P U$ e \# $\tau$ ) e precisão $s c d$, $M I I_{\text {proj }} \operatorname{com} \gamma=1$ ficou entre as melhores escolhas.

\begin{tabular}{|clll|rrrrrrr|}
\hline Solver & rtol & atol & \multicolumn{1}{c}{$h_{0}$} & scd & steps & accept & $\# \mathrm{~F}$ & \#Jac & \#LU & CPU \\
\hline \hline BIMD & $10^{-4}$ & $10^{-4}$ & $10^{-4}$ & 0.34 & 71 & 71 & 1693 & 71 & 71 & 0.0088 \\
& $10^{-7}$ & $10^{-7}$ & $10^{-7}$ & 3.34 & 138 & 138 & 4511 & 138 & 138 & 0.0224 \\
& $10^{-10}$ & $10^{-10}$ & $10^{-10}$ & 5.35 & 235 & 235 & 9669 & 235 & 235 & 0.0488 \\
GAMD & $10^{-4}$ & $10^{-4}$ & $10^{-4}$ & 0.39 & 39 & 39 & 2169 & 39 & 39 & 0.0088 \\
& $10^{-7}$ & $10^{-7}$ & $10^{-7}$ & 2.64 & 98 & 98 & 7167 & 98 & 98 & 0.0293 \\
& $10^{-10}$ & $10^{-10}$ & $10^{-10}$ & 3.84 & 179 & 179 & 18771 & 179 & 179 & 0.0742 \\
MEBDFI & $10^{-4}$ & $10^{-4}$ & $10^{-4}$ & -0.23 & 280 & 278 & 1246 & 27 & 27 & 0.0059 \\
& $10^{-7}$ & $10^{-7}$ & $10^{-7}$ & 3.34 & 650 & 648 & 2797 & 47 & 47 & 0.0137 \\
& $10^{-7}$ & $10^{-7}$ & $10^{-10}$ & 2.08 & 1393 & 1391 & 5624 & 85 & 85 & 0.0264 \\
PSIDE-1 & $10^{-4}$ & $10^{-4}$ & & -0.28 & 55 & 54 & 1403 & 42 & 220 & 0.0098 \\
& $10^{-7}$ & $10^{-7}$ & & 2.27 & 179 & 172 & 4103 & 83 & 464 & 0.0273 \\
& $10^{-10}$ & $10^{-10}$ & & 4.86 & 625 & 612 & 13751 & 115 & 964 & 0.0869 \\
RADAU & $10^{-4}$ & $10^{-4}$ & $10^{-4}$ & 0.19 & 98 & 97 & 850 & 95 & 98 & 0.0039 \\
& $10^{-7}$ & $10^{-7}$ & $10^{-7}$ & 2.51 & 289 & 288 & 2559 & 282 & 288 & 0.0127 \\
& $10^{-10}$ & $10^{-10}$ & $10^{-10}$ & 4.22 & 179 & 178 & 4281 & 169 & 179 & 0.0166 \\
\hline
\end{tabular}

Tabela 4.21: Problema CarAxis: Outros Solveres 
Comparação com outros métodos:

- Tome-se como base o resultado obtido para o $M I I_{\text {proj }} \operatorname{com} \gamma=1$.

- Comparando com a tabela acima, o scd foi melhor que todos os outros solveres, além disso

- A quantidade de cálculo de $\tau$ foi ao menos muitas vezes maior que a quantidade de cálculos de $f$ dos outros solveres. Por outro lado, este custo pode ser reduzido utilizando um método de integração numérica de passo múltiplo, como ilustrado abaixo, mantendo ainda bons resultados.

\begin{tabular}{|cccc|cccrrr|}
\hline Método & integrador & rtol & $\gamma$ & scd & scdY & $\# \tau$ & steps & $\# \mathrm{QR}$ & CPU \\
\hline \hline MII $_{\text {proj }}$ & ode113 & $10^{-4}$ & 1 & 1.29 & 0.65 & 1413 & 471 & 1413 & 0.056 \\
& & $10^{-7}$ & 1 & 4.33 & 3.41 & 2771 & 942 & 2771 & 0.064 \\
& & $10^{-10}$ & 1 & 7.51 & 5.99 & 4817 & 1606 & 4817 & 0.063 \\
\hline
\end{tabular}

Tabela 4.22: Problema CarAxis: $M I_{\text {proj }}$, rtol $=$ atol $=h_{0}$, ode113 


\subsection{Conclusões sobre as Implementações}

Sintetizam-se abaixo as conclusões dos experimentos numéricos:

- Foi possível realizar todas as simulações com ambas as escolhas de coordenadas complementares, $\mathrm{MII}_{\text {proj }}$ e $\mathrm{MII}_{\text {cond }}$.

- A transformação do Sistema Implícito em um Sistema Explícito foi vantajosa por permitir o uso de diferentes métodos de integração numérica, dependendo das características do problema, como ilustrado em Seção 4.6 - Andrews.

- De maneira geral o $\mathrm{MII}_{\text {proj }}$ apresentou melhores resultados e a escolha de $\gamma=0$ não foi boa, excetuando-se em especial a simulação do Seção 4.4 - SpaceShuttle.

- A utilização do parâmetro de convergência $\gamma=1$ mostrou-se uma boa primeira escolha para as simulações.

- O aumento excessivo de $\gamma$ pode gerar o fenômeno conhecido como stifness, dificultando a integração numérica por impor uma dinâmica muito rápida para o sistema em relação ao tamanho de passo de integração. Com isso, trazendo um maior custo computacional, não acompanhado necessariamente de um ganho de precisão compensatório (Seção 4.8 - CarAxis).

- Em muitos casos o $\mathrm{MII}_{\text {cond }}$ foi mais preciso que o $\mathrm{MII}_{\mathrm{proj}}$. Contudo, o refinamento do passo de integração para o MII $_{\text {proj }}$ foi mais vantajoso do que a imposição de uma tolerância menor no condicionamento para o $\mathrm{MII}_{\text {cond }}$, avaliando conjuntamente a precisão da solução obtida e o custo computacional. Como por exemplo: Seção 4.3 - Pêndulo.

- Para o $\mathrm{MII}_{\text {cond }}$, a imposição do parâmetro tol ${ }_{\text {cond }}$ muito justo tornou a simulação inviável para os problemas Seção 4.7 - TransAmp - e Seção 4.6 - Andrews.

- Não há indícios que a extensão do MII para sistemas variantes no tempo tenha prejudicado a performance do método.

- o artifício da transformação do sistema $M \frac{d}{d t}(w)=F(t, w)$, com $M$ uma matriz constante, num sistema semi-implícito utilizando decomposição em valores singulares, Seção 4.7 - TransAmp, originou resultados piores do que os obtidos pelos outros solveres, que lidam diretamente com essa classe de problemas.

A principal conclusão sobre os experimentos numéricos é:

O MII $_{\text {proj }}$ foi uma alternativa viável para a solução de sistemas implícitos com index baixos, mas ele mostrou-se uma ferramenta especialmente boa para problemas com index mais altos: Seção 4.7 - TransAmp e Seção 4.6 - Andrews. 


\section{Capítulo 5}

\section{Conclusões}

Neste trabalho aprimorou-se o método MII para aproximar soluções de DAEs, dadas como Sistemas Semi-Implícitos Quadrados (chamados aqui por simplicidade de Sistemas Implícitos),

$$
\left\{\begin{array}{l}
\dot{x}=f(x, u) \\
y=h(x) \equiv 0
\end{array}\right.
$$

através da integração numérica de um Sistema Explícito de equações diferenciais ordinárias,

$$
\left(\begin{array}{c}
\dot{x} \\
\dot{u}
\end{array}\right)=\tau(x, u)
$$

construído através do cálculos simbólicos e numéricos.

Inicialmente foi apresentada uma revisão da elementos da teoria de controle geométrico, definindo o método clássico por realimentação de estado estática (MI). Todavia, foi visto um exemplo simples no qual o erro relativo à variedade $\Gamma$ das soluções geradas pelo MI pode apresentar um comportamento indesejado.

Passou-se então para a definição do MII por injeção de saída generalizada, contornando esta dificuldade. O MII como em (2.55) é da forma:

$$
\left(\begin{array}{c}
\dot{x} \\
\dot{u}
\end{array}\right)=\tau(x, u) \text {, onde } T(x, u) \tau(x, u)=\left(\begin{array}{c}
\frac{\partial \hat{x}}{\partial x}(f(x, u)) \\
-\gamma Y^{(\rho)}(x, u)
\end{array}\right) \text {, com } T(x, u) \text { não singular }
$$

observando que:

- a função $Y^{(\rho)}(x, u) \doteq\left(Y^{(\rho-1)}(x), y^{(\rho)}(x, u)\right)$ é construída simbolicamente através de derivadas sucessivas das saída do Sistema Implícito (vide $(2.23)$ ), de forma que $Y^{(\rho-1)}(x)$ não depende de $u$ e $y^{(\rho)}(x, u)$ depende de $u$;

- $\hat{x}(x)$ são coordenadas complementares quaisquer, escolhidas para que a aplicação $\Psi(x, u)=$ $\left(\hat{x}(x), Y^{(\rho-1)}(x), y^{(\rho)}(x, u)\right)$ seja uma transformação de coordenadas suave. Ressaltando que na Seção 2.2 prova-se a existência de tais coordenadas complementares suaves desde que $\frac{\partial Y^{(\rho-1)}}{\partial x}$ possua posto máximo numa vizinhança;

- $T(x, u)$ é uma notação para a matriz jacobiana $\frac{\partial \Psi(x, u)}{\partial x, u}$.

O próximo passo foi verificar que as soluções do Sistema Implícito evoluem sobre uma subvariedade $\Gamma \doteq\left\{(x, u) ; Y^{(\rho)}(x, u)=0\right\}$.

Os teoremas discutindo a proximidade entre as soluções do MII e do Sistema Implícito foram aperfeiçoados e suas demonstrações tornadas mais claras. Provou-se com o Teorema 2.25 que a distância $\left\|Y^{(\rho)}(x, u)\right\|$ entre as soluções do MII e $\Gamma$ decresce exponencialmente. 
Na sequência do trabalho, tratou-se da obtenção pontual de coordenadas complementares, empregando técnicas de análise numérica com objetivo de melhorar a precisão e a performance do algoritmo.

Foram levantadas e testadas algumas novas opções para escolhas numéricas de $\hat{x}(x)$, das quais o MII $_{\text {cond }}$ e o MII $_{\text {proj }}$, respectivamente nas Seções 3.3 e 3.3, obtiveram os melhores resultados.

A ideia principal do MII $_{\text {cond }}$ é utilizar uma escolha linear de coordenadas complementares $\hat{x}(x) \doteq$ $K x$ e balanceamentos nas coordenadas $Y^{(\rho)}$, recalculados quando o número de condicionamento dos blocos diagonais ultrapassar uma tolerância tol ${ }_{\text {cond }}$. Com isso, buscou-se que a eliminação de Gauss envolvendo $T(x, u)$ fosse sempre numericamente estável.

O conceito do $\mathrm{MII}_{\mathrm{proj}}$ é tomar uma base para o complemento ortogonal do espaço gerado pelas linhas de $\frac{\partial Y^{(\rho-1)}}{\partial x}$, denotada matricialmente por $R(x)$, em cada um dos pontos $x$ no decorrer da simulação. Além disso, aproveita-se a estrutura especial da matriz inversa de $T(x, u)$, descrita em (3.8), juntamente com uma interpretação geométrica da expressão obtida. Repete-se aqui por comodidade a estrutura obtida para o MII $_{\text {proj }}$ como em (3.11):

$$
\left\{\begin{array}{l}
\dot{x}=\tau_{x}(x, u)=\operatorname{proj}_{[R]}(f(x, u))-\gamma \operatorname{inc}_{\left[Y_{x}\right]}\left(Y^{(\rho-1)}(x)\right) \\
\dot{u}=\tau_{u}(x, u)=\left(\frac{\partial y^{(\rho)}}{\partial u}\right)^{-1}\left(-\gamma y^{(\rho)}(x, u)-\frac{\partial y^{(\rho)}}{\partial x} \dot{x}\right)
\end{array}\right.
$$

Outro fato importante é a interpretação geométrica simples da expressão obtida para o campo vetorial $\tau_{x}$, composto pela soma de dois vetores perpendiculares:

- $\operatorname{proj}_{[R]}(f(x, u))$ : projeção ortogonal do vetor $f(x, u)$ sobre o espaço tangente à superfície definida por $\left\{\bar{x} \in \mathbb{R}^{n} ;\left\|Y^{(\rho-1)}(\bar{x})\right\|=\varepsilon\right\}$, no ponto $x$, com $\varepsilon \doteq\left\|Y^{(\rho-1)}(x)\right\|$.

- $-\gamma \operatorname{inc}_{\left[Y_{x}\right]}\left(Y^{(\rho-1)}(x)\right)$ : inclusão no espaço de estados de uma componente corrigindo o erro $Y^{(\rho-1)}(x)$ em relação à variedade, com velocidade multiplicada por $\gamma$.

As simulações dos três primeiros problemas mostram que ambas escolhas numéricas de coordenadas complementares são aplicáveis. Com os bechmarks, constatou-se que o MII $_{\text {cond }}$ foi inviável em alguns casos, devido ao tempo de processamento muito extenso, exigindo o ajuste do parâmetro tol $\mathrm{l}_{\text {cond }}$. Por outro lado, o $\mathrm{MII}_{\text {proj }}$ apresentou melhores resultados, sendo que parâmetro de convergência $\gamma=1$ com integrador ode 45 mostrou-se uma boa primeira escolha em todos os casos.

Ademais, a liberdade de escolha para o integrador numérico, como por exemplo Runge-Kutta ou Adams Bash-forth, mostrou-se uma boa ferramenta para o ajuste das características da solução gerada dependendo da necessidade.

Conclui-se por fim, ao comparar com os demais solveres do [MM08], que o o MII $_{\text {proj }}$ é uma alternativa válida para aproximação numérica desta classe de problemas, em especial para sistemas com index alto (maior ou igual a 3 ).

\subsection{Sugestões para Pesquisas Futuras}

Como sugestões para futuras pesquisas e implementação, ressaltam-se:

- Emprego de estratégia numérica para obter maior robustez na identificação do anulamento simbólico da expressão $\frac{\partial \bar{h}}{\partial u}$, conforme comentário ao final da Seção 3.1.

- Definição de um método para uma busca de solução inicial $\left(x_{0}, u_{0}\right)$ tal que $\left(x_{0}, u_{0}\right)$ pertença à $\Gamma$.

- Implementação usando um pacote de cálculo simbólico em Fortran.

- Generalização do método para lidar diretamente com problemas da forma $M(w) w^{\prime}=F(t, w)$, ou ainda $F\left(t, w, w^{\prime}\right)=0$. 
- Generalização do método para tratar de sistemas semi-implícitos não necessariamente quadrados, i.e., $\operatorname{com} y \in \mathbb{R}^{m}$ e $u \in \mathbb{R}^{l}, \operatorname{com} l \geq m$. 


\section{Capítulo 6}

\section{Apêndice}

Neste capítulo incluem-se os códigos-fonte desenvolvidos em Matlab. Inicialmente estão as rotinas para construção das simulações e rotinas auxiliares, descritas na Seção 3.6, incluem-se depois o códigos-fonte dos problemas simulados.

\subsection{Código-Fonte Construção da Implementação}

Arquivo: ConstroiFuncao.m

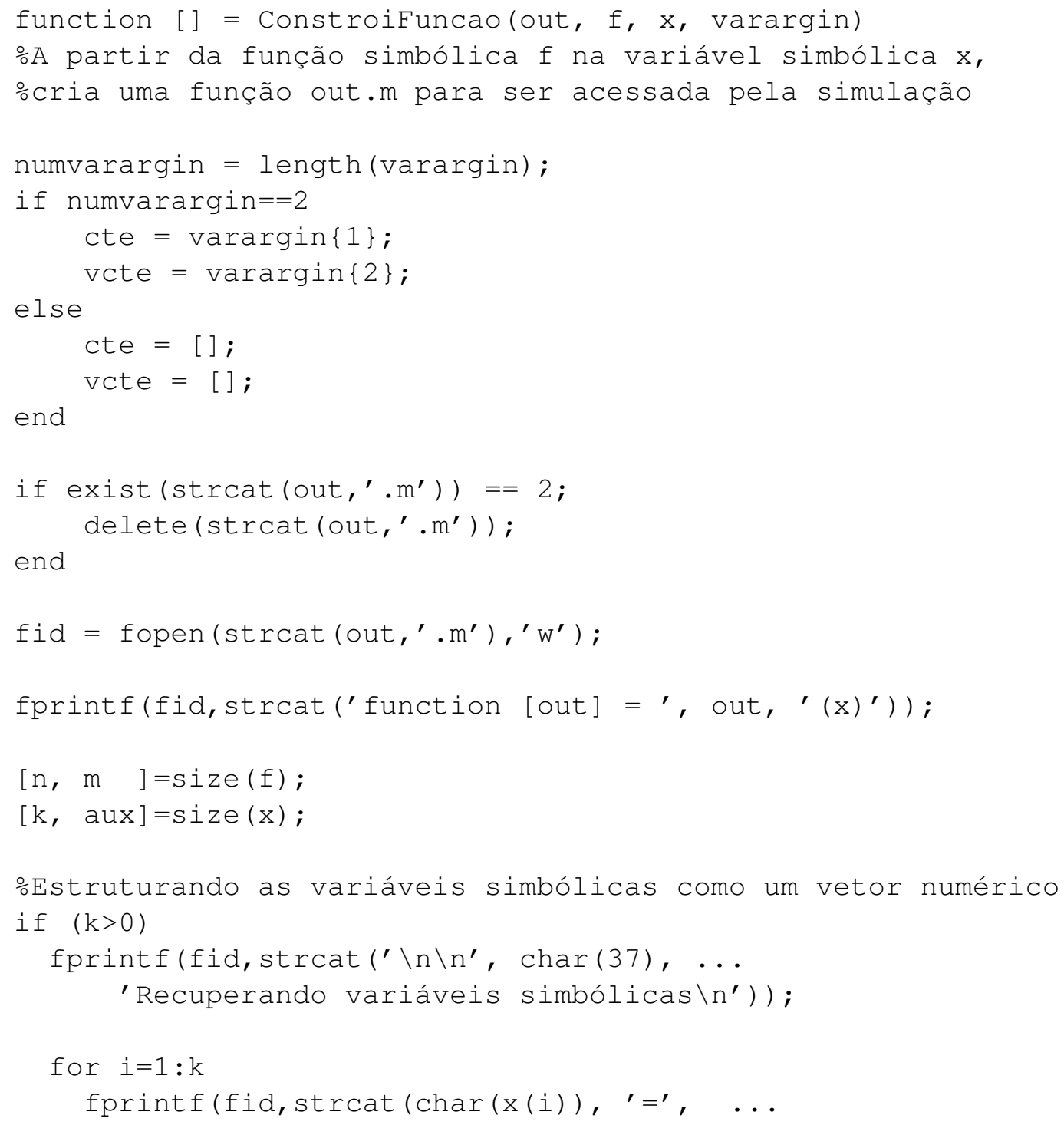


end

$$
\left.\left.{ }^{\prime} \mathrm{x}\left({ }^{\prime}, \operatorname{num} 2 \operatorname{str}\left(i,{ }^{\prime} \frac{\mathrm{o}}{\mathrm{d}} \mathrm{d}^{\prime}\right),{ }^{\prime}\right)^{\prime},{ }^{\prime}, ; \backslash \mathrm{n}^{\prime}\right)\right) \text {; }
$$

end

oAtribuindo valor às ctes

[k, aux ] =size (cte);

if $(k \sim=0)$

fprintf(fid, strcat (' $\backslash n \backslash n^{\prime}, \operatorname{char}(37), \ldots$

'Atribuindo valores às constantes $\left.\backslash n^{\prime}\right)$ );

for $i=1: k$

if $(\operatorname{vcte}(i)==180 / \mathrm{pi})$

fprintf(fid, strcat (char (cte(i)), $\quad{ }^{\prime}={ }^{\prime}, \ldots$

'180/pi', '; $\left.\left.\backslash \mathrm{n}^{\prime}\right)\right)$;

else

fprintf(fid, strcat (char (cte(i)), ${ }^{\prime}={ }^{\prime}, \ldots$

num2str(vete(i), ' $\left.\left.\left.\frac{\circ}{\circ} 20 f^{\prime}\right), \prime^{\prime} ; \backslash n^{\prime}\right)\right)$;

end

end

end

oEscrevendo função

fprintf(fid, strcat (' $\backslash n \backslash n^{\prime}, \operatorname{char}(37), \ldots$

'Escrevendo a função $\left.\backslash \mathrm{n}^{\prime}\right)$ ));

fprintf(fid, strcat ('out', ''=zeros (', ...

num2str(n), ',', num2str(m), '); $\left.\left.\backslash \mathrm{n} \backslash \mathrm{n}^{\prime}\right)\right)$;

for $i=1: n$

for $j=1: m$

if isnumeric( $f(i, j))$

fprintf(fid, strcat ('out', ' (', num2str(i), . .

$\left.\left.\left.{ }^{\prime},{ }^{\prime}, \operatorname{num} 2 \operatorname{str}(j),{ }^{\prime}\right)={ }^{\prime}, \operatorname{num} 2 \operatorname{str}\left(f(i, j),{ }^{\prime} \circ 20 f^{\prime}\right),{ }^{\prime} ; \backslash n^{\prime}\right)\right)$;

else

fprintf(fid, strcat ('out', ' (', num2str(i), ...

$\left.\left.\left.{ }^{\prime},{ }^{\prime}, \operatorname{num} 2 \operatorname{str}(j),{ }^{\prime}\right)={ }^{\prime}, \operatorname{char}(f(i, j)),{ }^{\prime} ; \backslash \mathrm{n}^{\prime}\right)\right)$;

end

end

end

status $=$ fclose $($ fid $) ;$

Arquivo: Derivacoes_Sucessivas.m

function[p, y, Yx, yp, ypx, ypu] = Derivacoes_Sucessivas(f, $h, \mathrm{x}, \mathrm{u})$ oCria funcoes atraves de derivadas sucessivas da saida

oInicializando variáveis auxiliares

$\mathrm{p}=[] ; \mathrm{Y}=[] ; \mathrm{Yx}=[] ; \quad \mathrm{yp}=[] ; \operatorname{ypx}=[] ; y p u=[]$;

oCalculando o tamanho dos vetores 


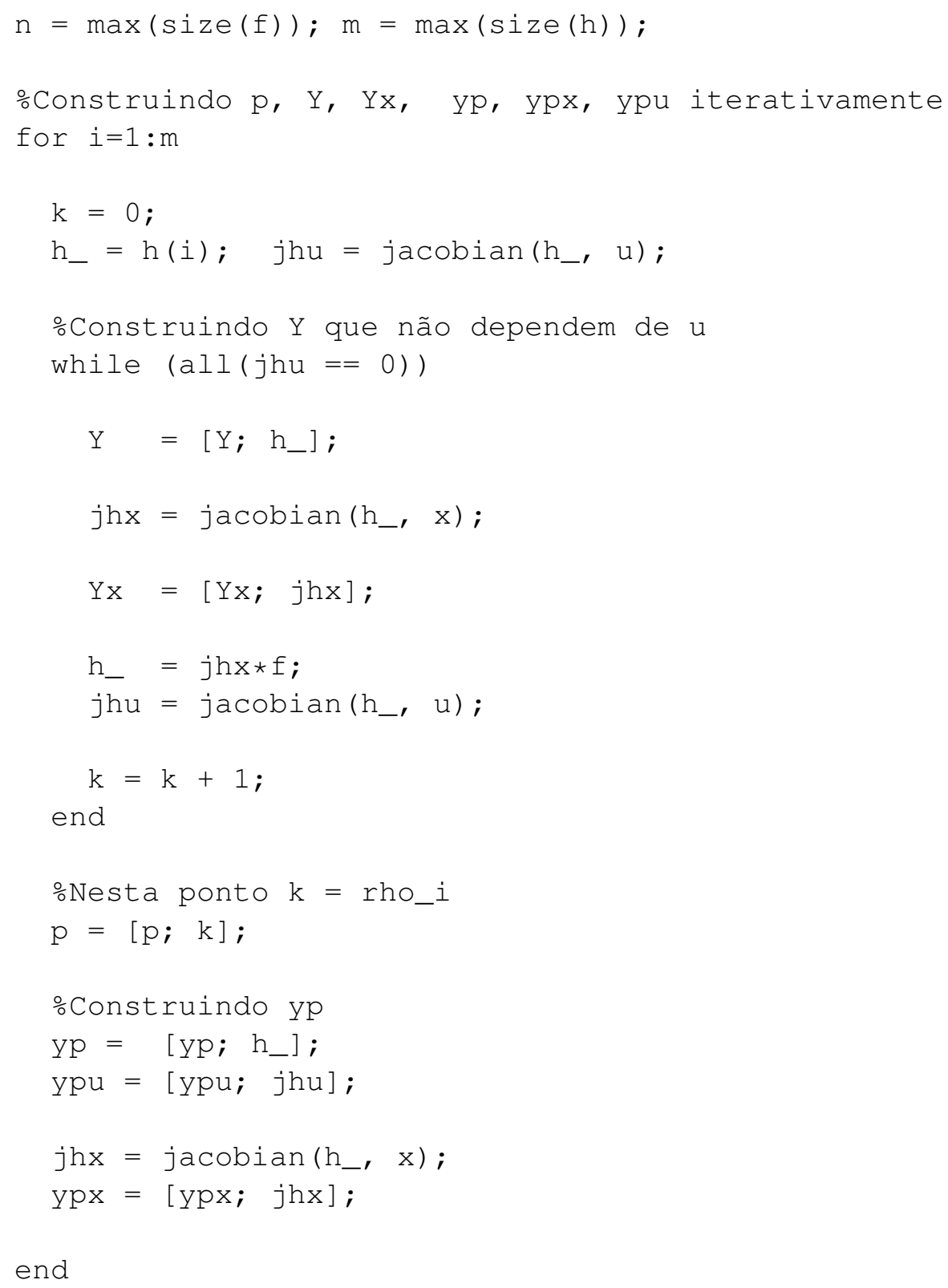

end

Arquivo: ConstroiImplementacao.m

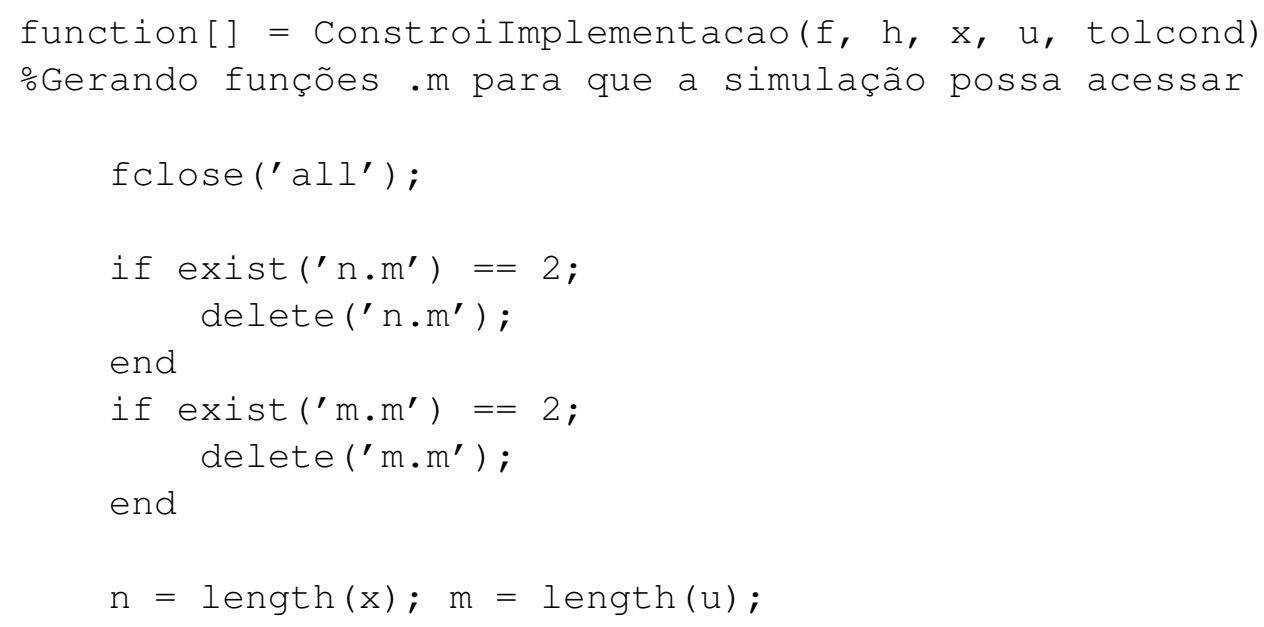


oSempre faz a simulação na generalização variante no tempo

if $\left(\operatorname{char}(x(1))=={ }^{\prime} t^{\prime}\right)$

$\mathrm{tx}=\mathrm{x}$;

$\mathrm{n}=\mathrm{n}-1$;

else

syms t;

$t x=[t ; x]$;

end

\%Obtendo a transformação de coordenadas por derivações sucessivas $[p, y, Y t x, y p, y p t x, y p u]=$ Cria_Funcoes_Simbolicas $([1 ; f], h, t x, u)$;

if isempty (Ytx)

$\mathrm{Yt}=\mathrm{YtX}(:, 1)$;

$\mathrm{YX}=\operatorname{YtX}(:, 2:(\mathrm{n}+1))$;

else

Yt $=[] ;$

$\mathrm{YX}=[] ;$

end

ypt $=\operatorname{yptx}(:, 1) ;$

$\operatorname{ypx}=\operatorname{yptx}(:, 2:(\mathrm{n}+1))$;

$t x u=[t x ; u] ;$

oConstruindo funções para a transformação e suas derivadas

Constroifuncao('Y', Y, txu);

Constroifuncao('yp', yp, txu);

Constroifuncao('Yt', Yt, txu);

Constroifuncao('YX', YX, tXu);

Constroifuncao('ypt', ypt, txu);

Constroifuncao('ypx', ypx, txu);

Constroifuncao('ypu', ypu, txu);

oConstruindo as funções de definição do problema

Constroifuncao('f', f, txu);

Constroifuncao('h', h, txu);

Constroifuncao('n', n, []); Constroifuncao('m', m, []);

Constroifuncao ('dimout', $\mathrm{n}+\mathrm{m}, \mathrm{l}]$ );

Constroifuncao('p', $\operatorname{sum}(\mathrm{p}),[])$;

Constroifuncao ('tolcond', tolcond, []);

pause (3);

global rl;

$\mathrm{r} 1$ = eye $(\mathrm{n})$; 
Arquivo: Complemento_Ortogonal.m

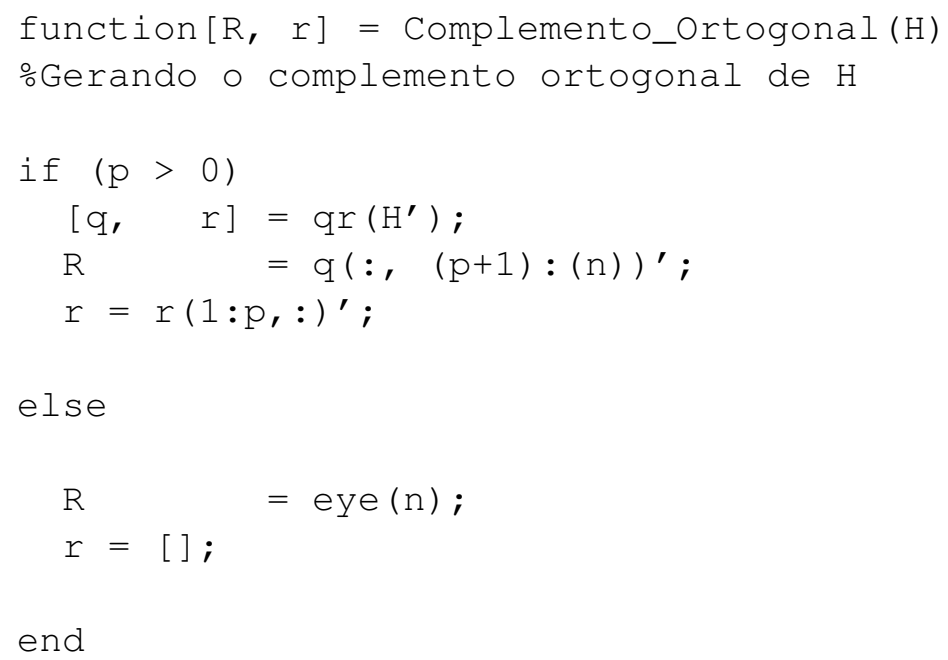

Arquivo: ParametrosBalanceamento.m

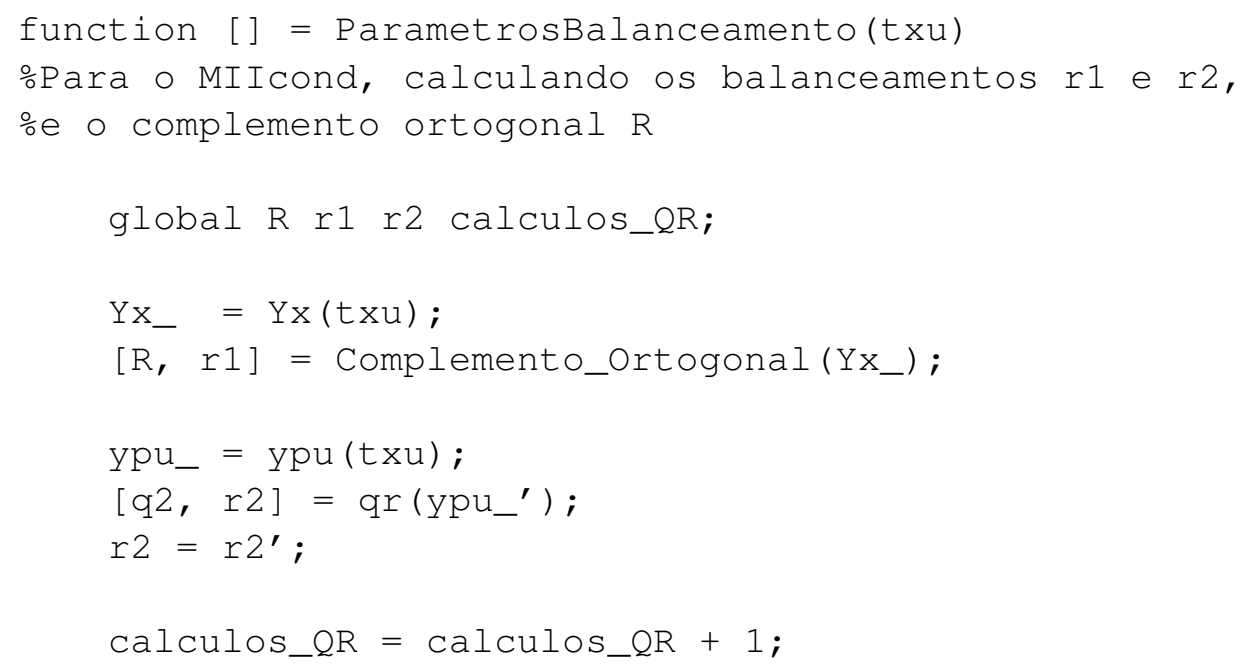

Arquivo: SimulinkAdaptado.m

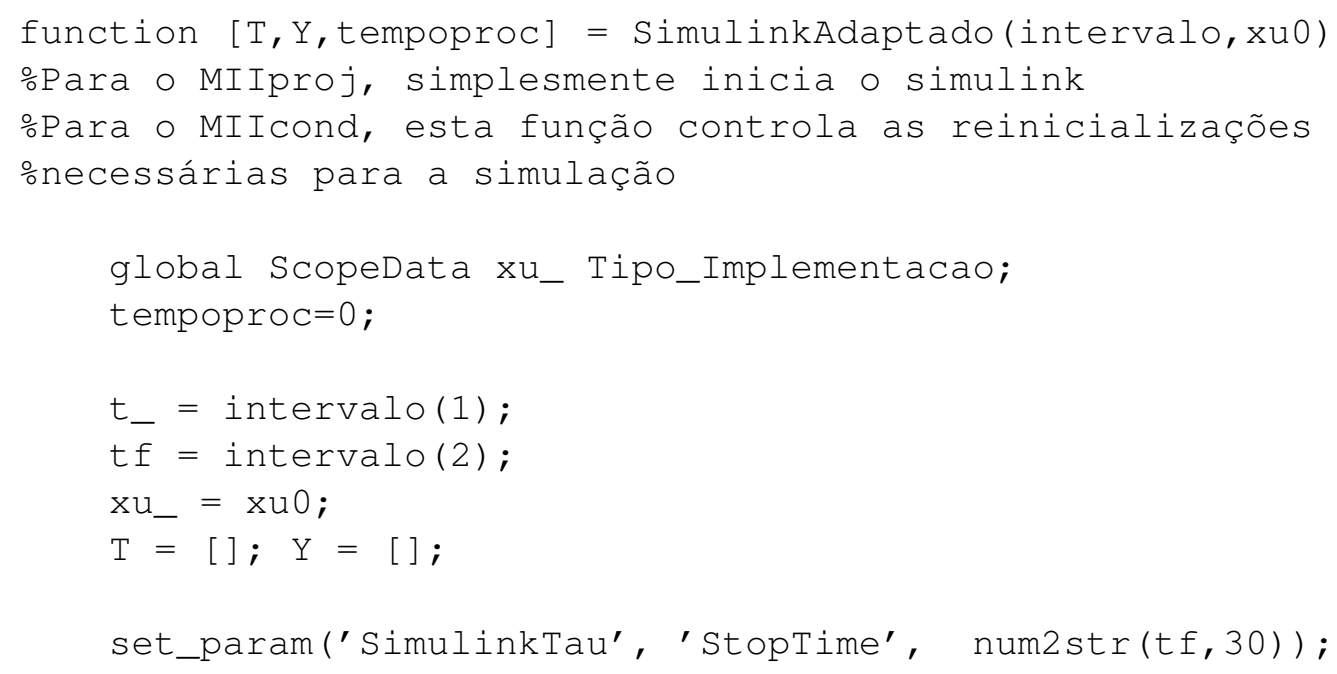


while $t_{-}<$tf

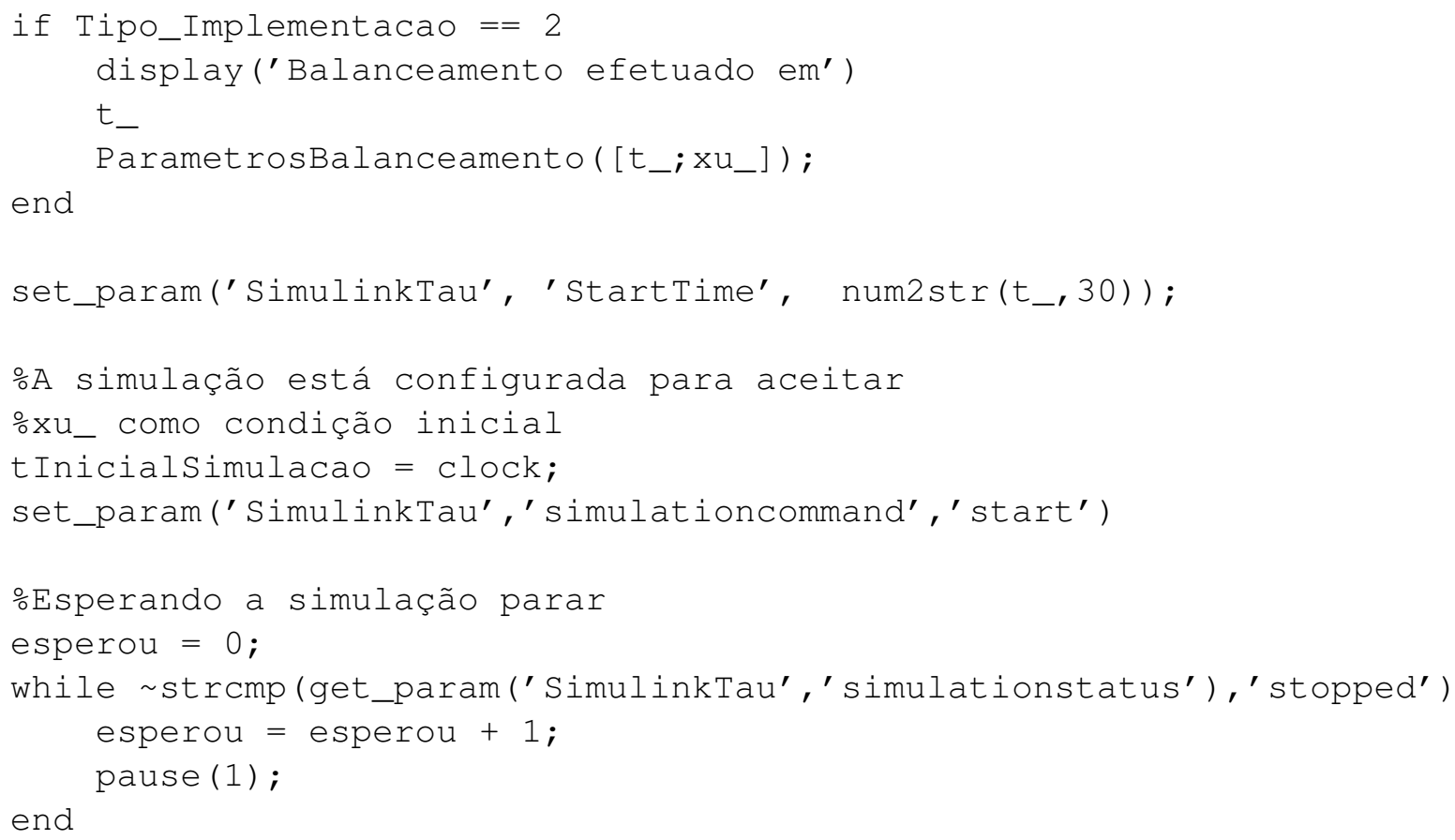

Arquivo: Avalia_tau.m

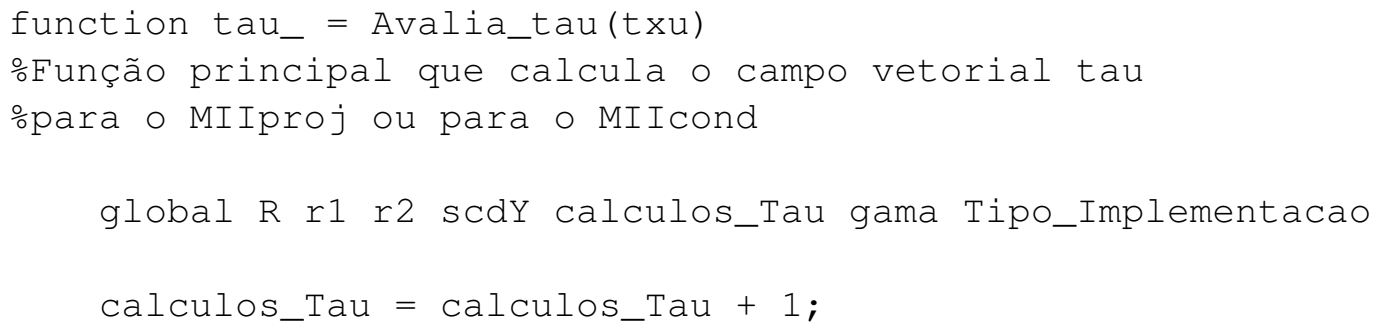


tau2 $=$ ypu_l $\left(-y_{-} x_{-}\right.$taul - gama $*$ yp_ - ypt_ $)$;

end

scdY $=\max ($ scdY, auxnorm $)$;

tau $u_{-}=[\operatorname{tau} 1 ; \operatorname{tau} 2] ;$

return

\subsection{Código-Fonte MatrizCond}

Arquivo: Problema_MatrizCond.m

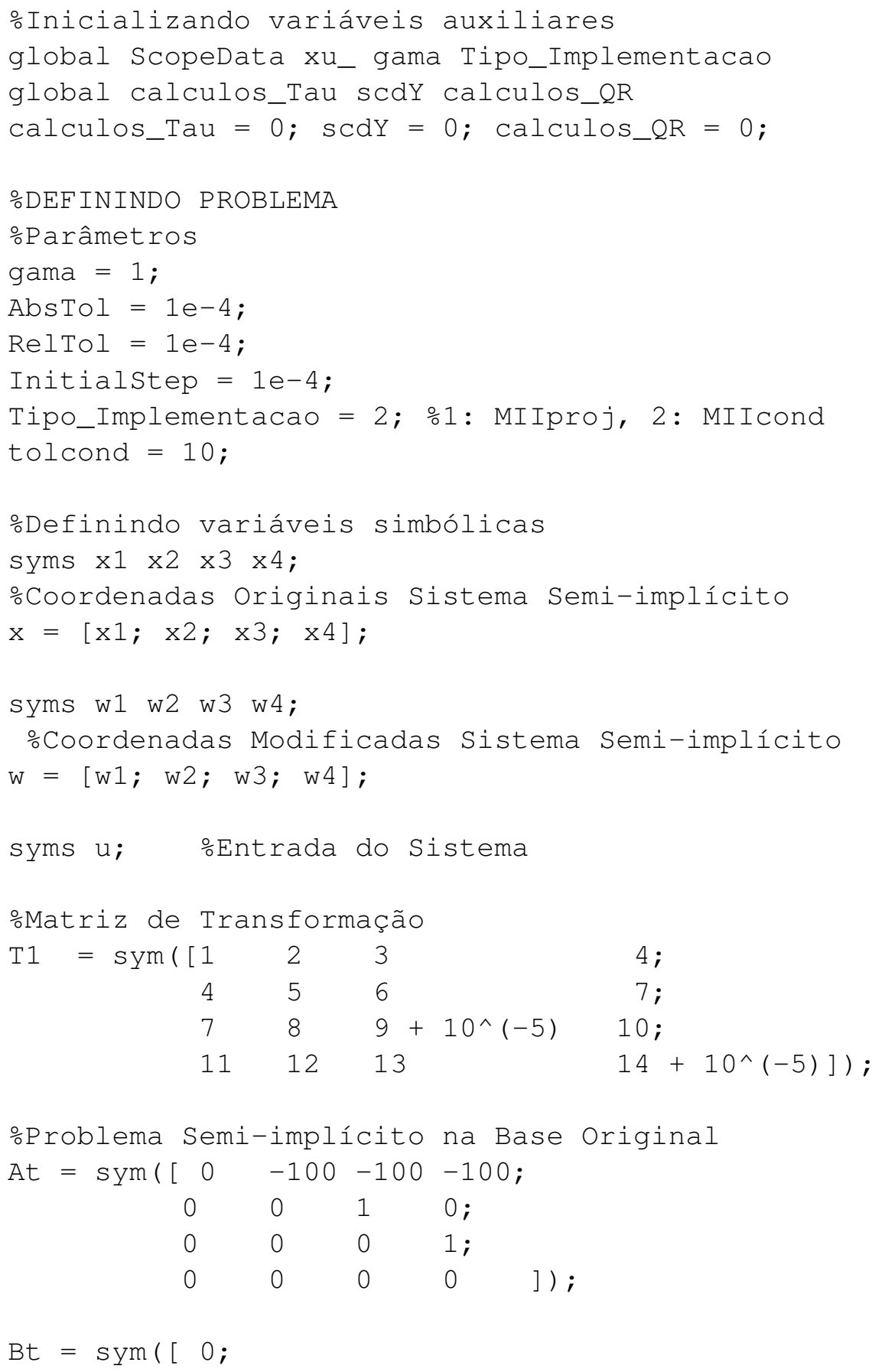




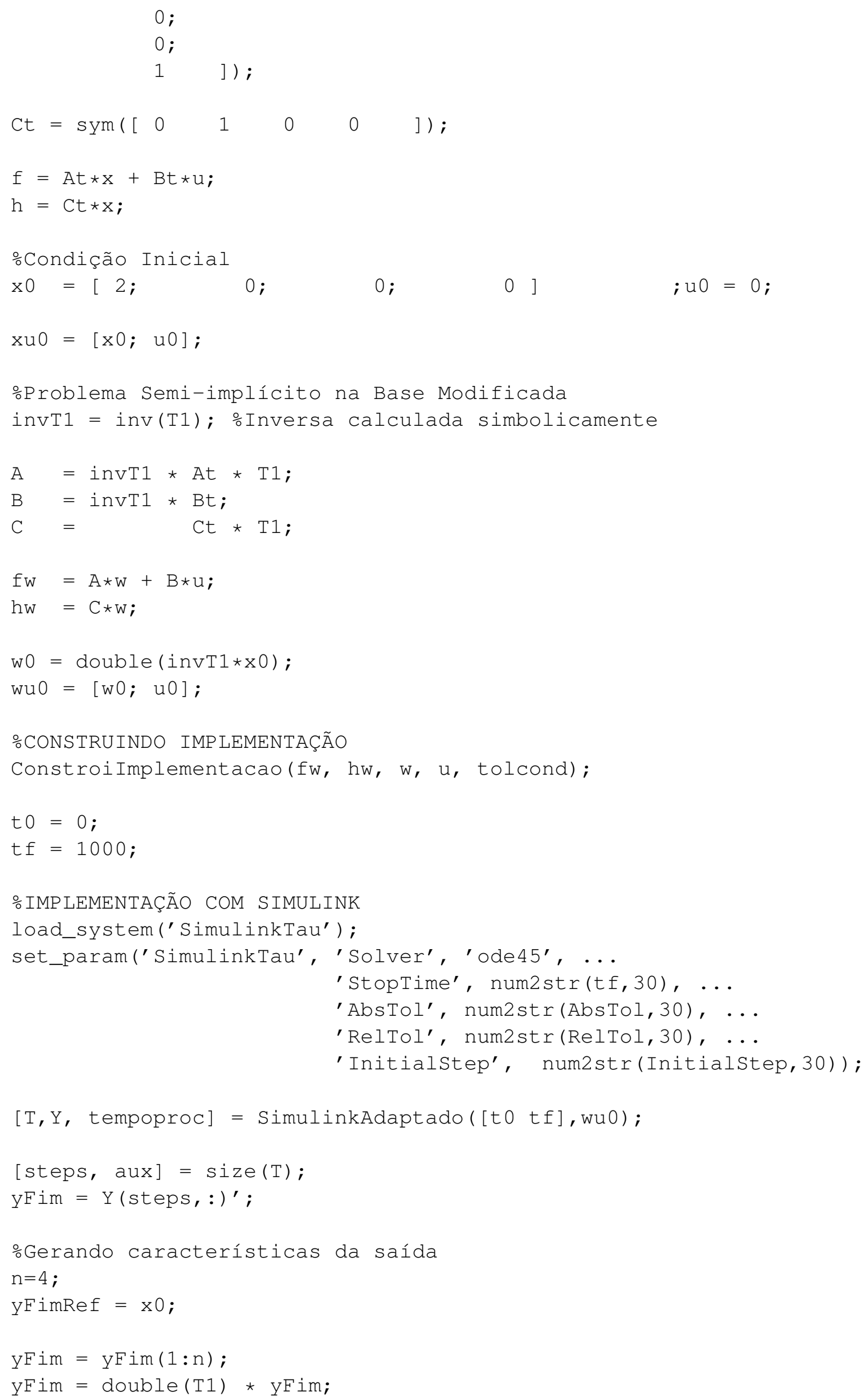


scd $=a b s(y F i m-y F i m R e f) ;$

$\mathrm{scd}=-\log 10(\max (\mathrm{scd}))$;

$\operatorname{scdY}=-\log 10(\max (\operatorname{scdY})) ;$

if Tipo_Implementacao $==1$

calculos_QR = calculos_Tau;

end

Resultados $=[$ scd scdY calculos_Tau steps calculos_QR tempoproc];

num2str(Resultados)

\subsection{Código-Fonte Pêndulo}

Arquivo: Problema_Pendulo.m

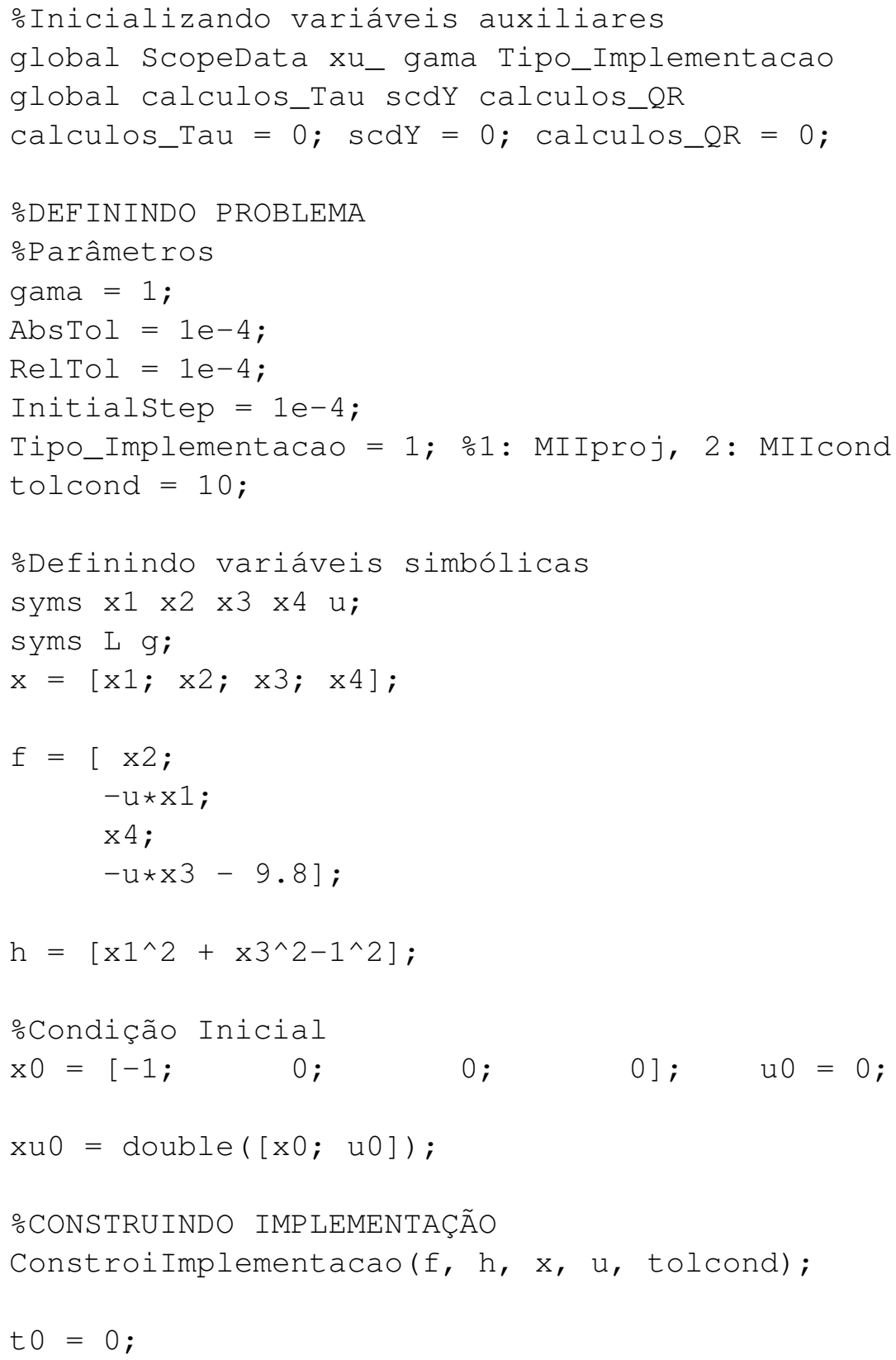




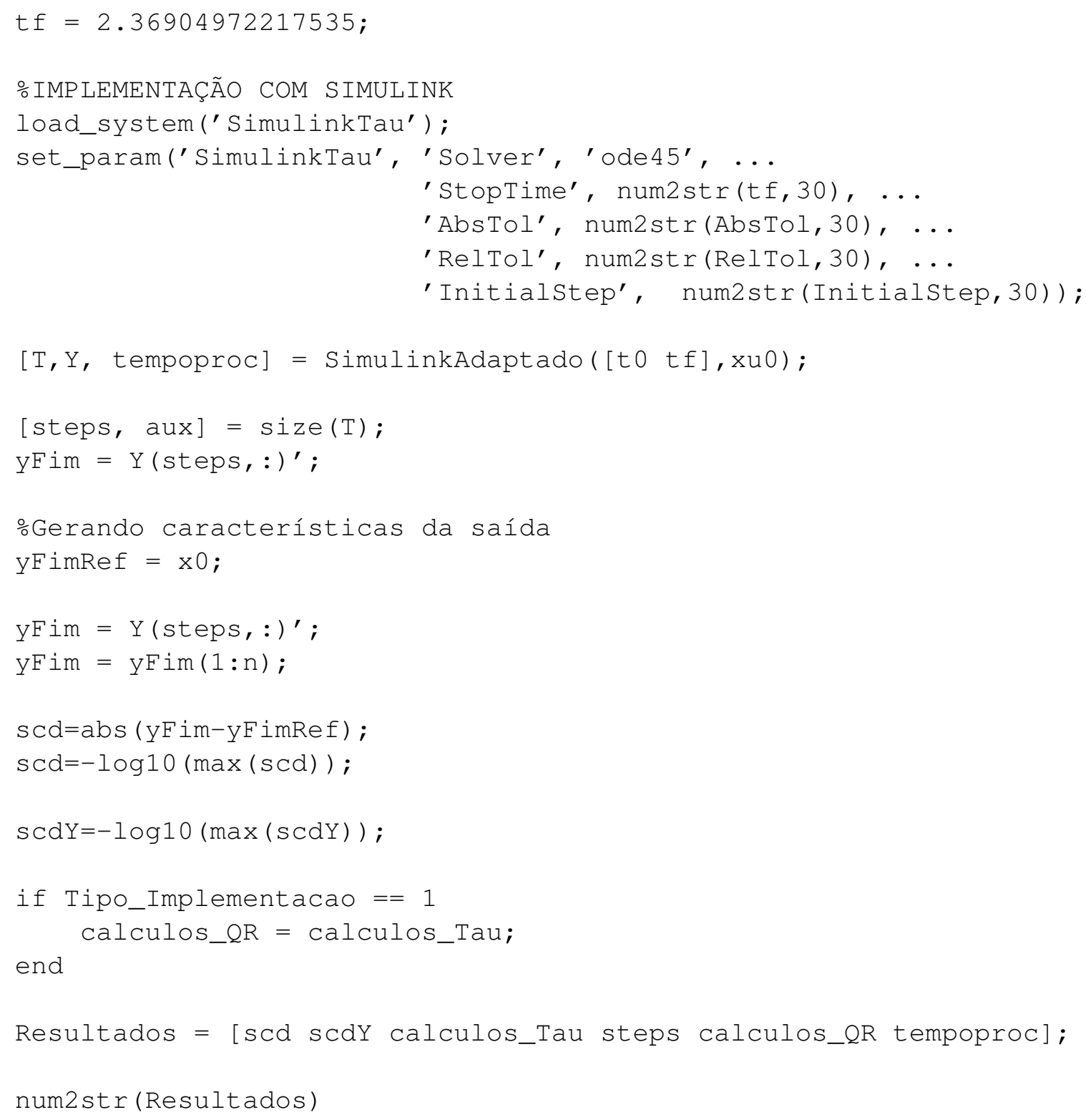

\subsection{Código-Fonte SpaceShuttle}

Arquivo: Problema_SpaceShuttle.m

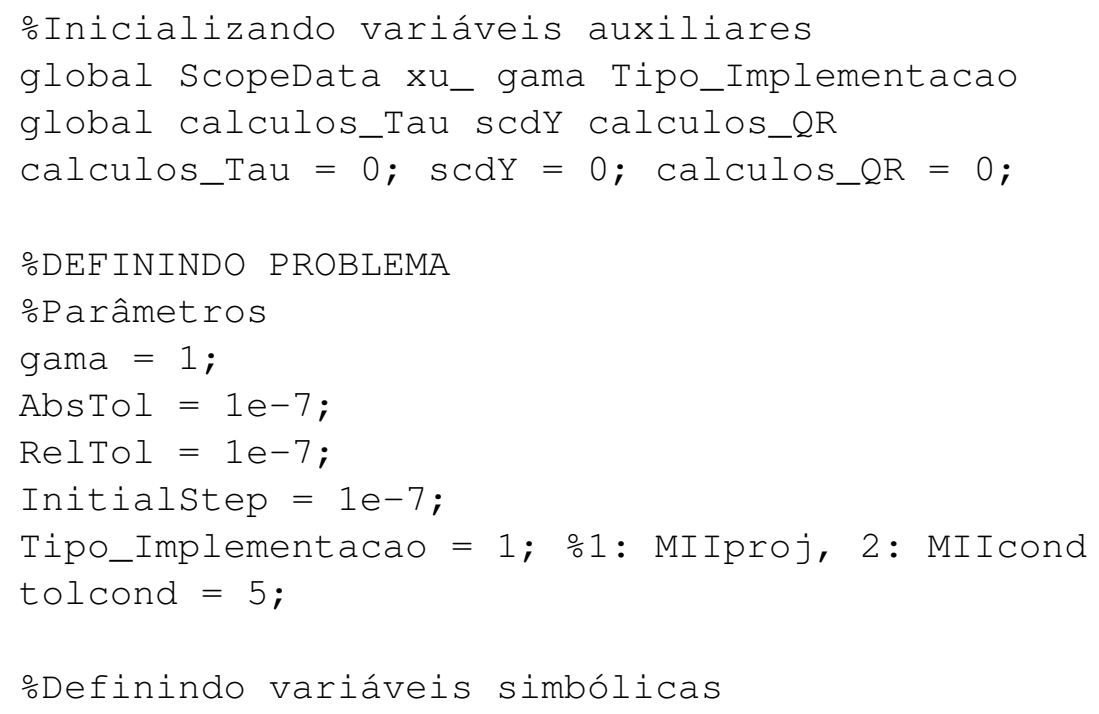


syms t $\mathrm{H}$ xi lam VR gam A alp bet

syms betp alpp betpp alppp delta

-Atribundo valores às constantes

$\mathrm{ae}=20902900$;

$\mathrm{mu}=0.14076539 \mathrm{e} 17$;

OmegaE $=0.72921159 \mathrm{e}-04$;

$\mathrm{m}=2$. 890532728 ;

$\mathrm{a}=0.002378$;

$\mathrm{b}=23800$;

$\mathrm{c}=180 / \mathrm{pi}$;

$\mathrm{S}=1$;

$\mathrm{r}=\mathrm{H}+\mathrm{ae}$;

$g=m u /(r * r)$;

$f=[V R * \sin (g a m) ;$

$\mathrm{VR} * \cos ($ gam $) * \sin (\mathrm{A}) /(\mathrm{H}+\mathrm{ae}) / \cos (\mathrm{lam}) ;$

$\mathrm{VR} * \cos ($ gam $) * \cos (\mathrm{A}) /(\mathrm{H}+\mathrm{ae})$;

$-1 / 2 \star a * \exp (-\mathrm{H} / \mathrm{b}) \star\left(1 / 25+1 / 100000 * \mathrm{c}^{\wedge} 2 * a l \mathrm{p}^{\wedge} 2\right) \star \ldots$

$\mathrm{S} * \mathrm{VR} \wedge 2 / \mathrm{m}-\mathrm{mu} /(\mathrm{H}+\mathrm{ae})^{\wedge} 2 * \sin (\mathrm{gam})-\mathrm{Omegat}^{\wedge} 2 *(\mathrm{H}+\mathrm{ae}) * \ldots$

$\cos (1 a m) *(\sin (l a m) * \cos (A) * \cos (g a m)-\cos (l a m) * \sin (g a m))$;

$1 / 200 * a * \exp (-\mathrm{H} / \mathrm{b}) * \mathrm{C} * \mathrm{al} \mathrm{p} * \mathrm{~S} * \mathrm{VR} * \cos ($ bet $) / \mathrm{m}+\cos (\mathrm{gam}) * \ldots$

$\left(\mathrm{VR}^{\wedge} 2 /(\mathrm{H}+\mathrm{ae})-\mathrm{mu} /(\mathrm{H}+\mathrm{ae})^{\wedge} 2\right) / \mathrm{VR}+2 \star$ OmegaE $\star \cos (\mathrm{lam}) \star \ldots$

$\sin (A)+O m e g a E^{\wedge} 2 *(H+a e) * \cos (l a m) *(\sin (l a m) * \cos (A) * \ldots$

$\sin ($ gam $)+\cos (1 \mathrm{am}) * \cos ($ gam $)) / \mathrm{VR}$;

$1 / 200 * a * \exp (-H / b) * C * a l p * S * V R * \sin ($ bet $) / m / \cos (g a m)+\ldots$

$\mathrm{VR} * \cos (\mathrm{gam}) \star \sin (\mathrm{A}) * \tan (\mathrm{lam}) /(\mathrm{H}+\mathrm{ae})-2 *$ OmegaE $* \ldots$

$(\cos (\operatorname{lam}) * \cos (A) * \tan (g a m)-\sin (\operatorname{lam}))+\ldots$

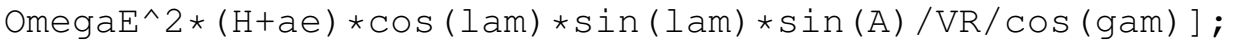

$\mathrm{x}=[\mathrm{H} ;$

xi;

lam;

$\mathrm{VR}$;

gam;

A] ;

$\mathrm{u}=[$ bet;

alp];

$\mathrm{y} 1=\left((180 / \mathrm{pi}) * \mathrm{gam}+1+9 *(\mathrm{t} / 300)^{\wedge} 2\right) ;$

$\mathrm{y}^{2}=\left((180 / \mathrm{pi}) * \mathrm{~A}-45-90 *(\mathrm{t} / 300)^{\wedge} 2\right) ;$

$\mathrm{h}=[\mathrm{y} 1 ; \mathrm{y} 2] ;$

oCondição Inicial

$\mathrm{x} 0=[100000$;

0 ;

0 ;

12000 ;

$-\mathrm{pi} / 180$; 


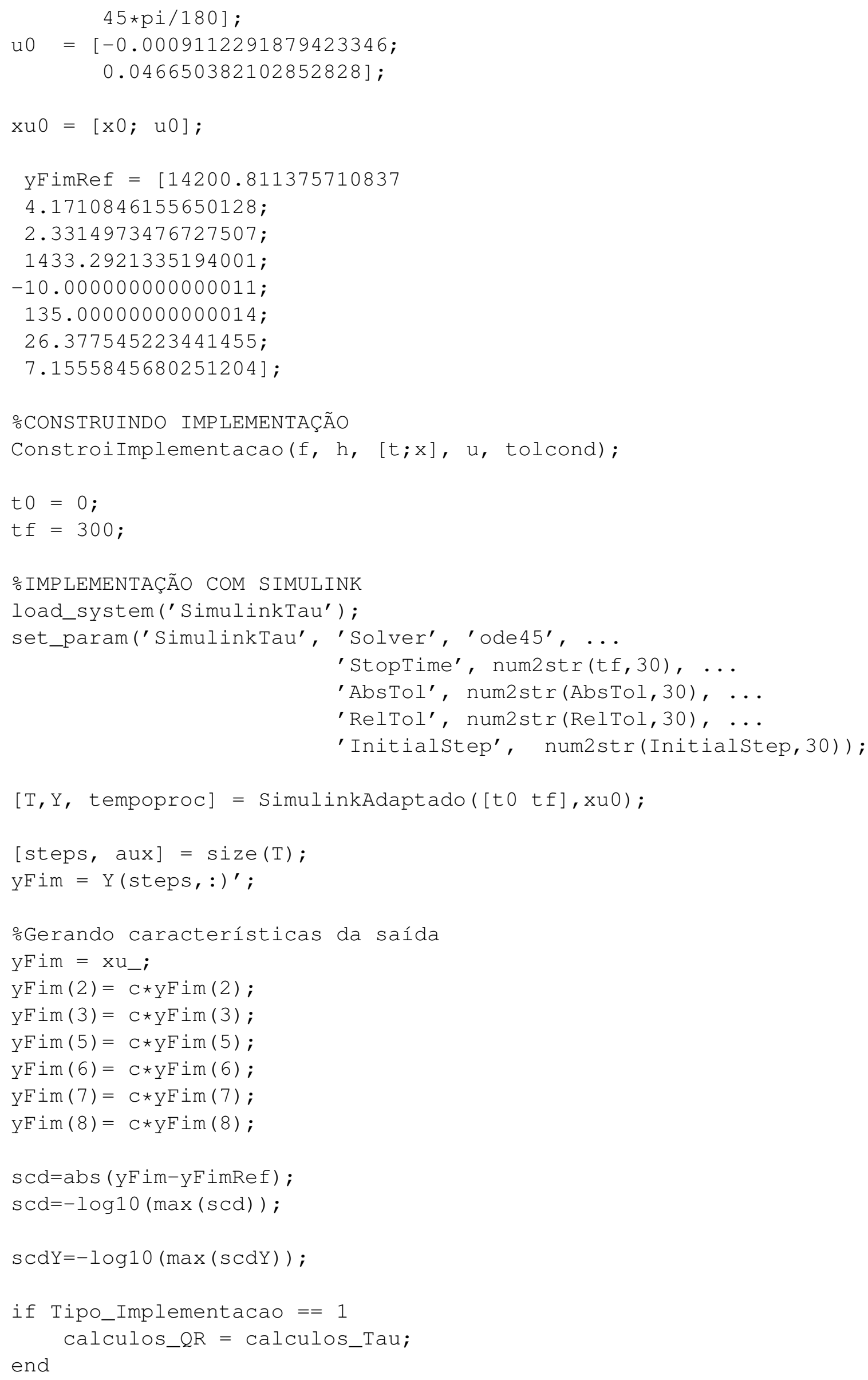


Resultados $=[$ scd scdY calculos_Tau steps calculos_QR tempoproc]; num2str (Resultados)

\subsection{Código-Fonte Chemakzo}

Arquivo: Problema_Chemakzo.m

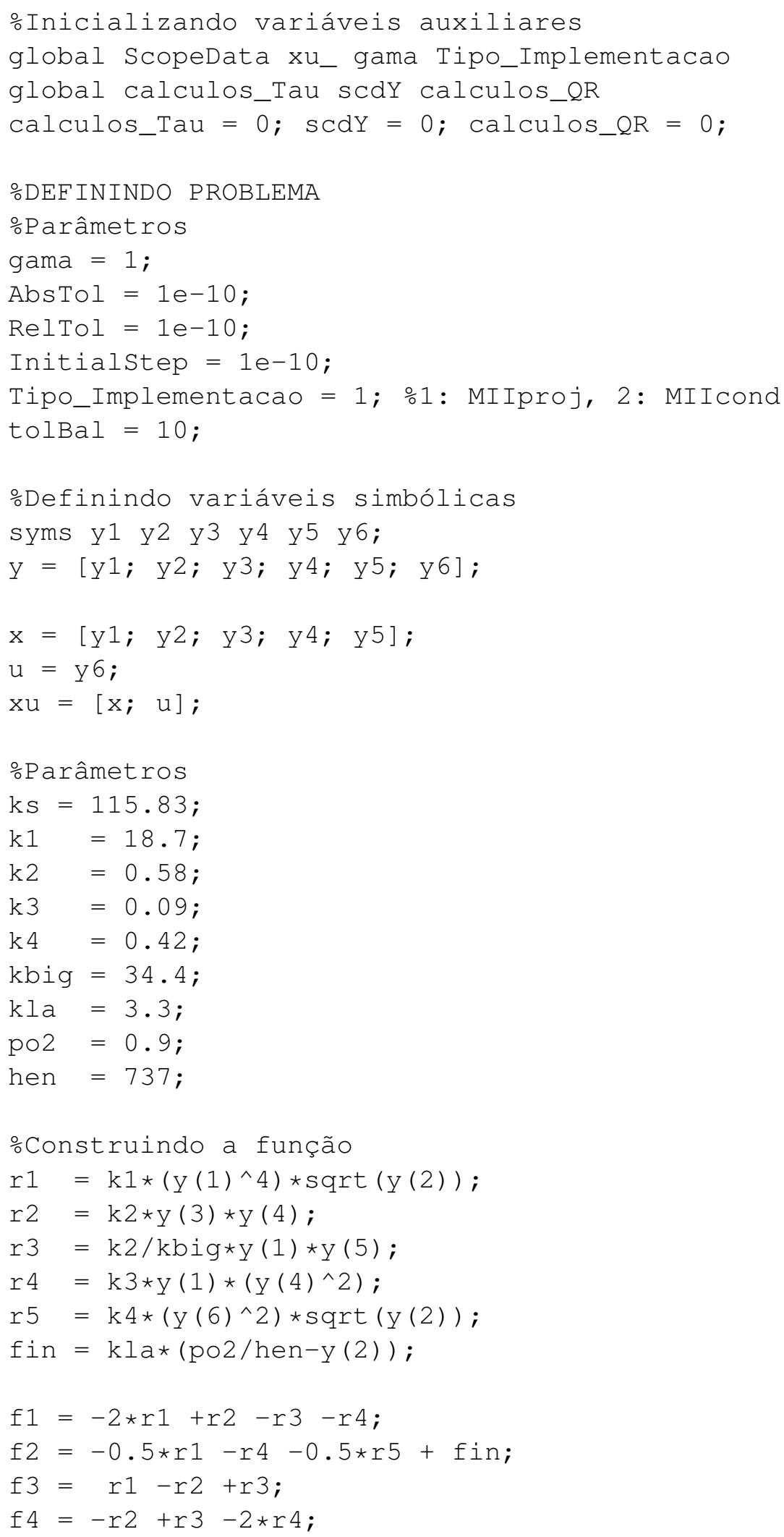




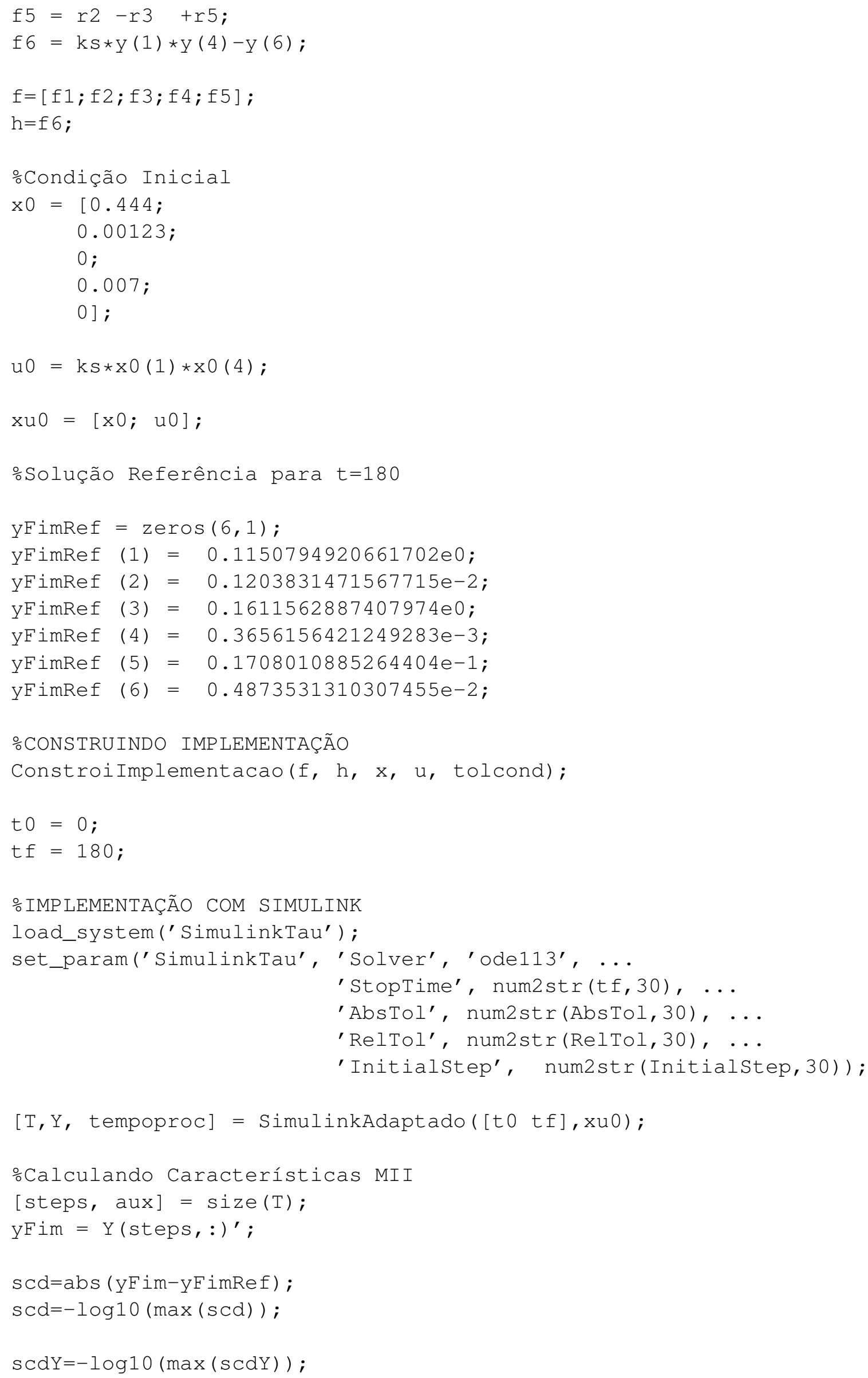


if Tipo_Implementacao $==1$

calculos_QR = calculos_Tau;

end

Resultados $=[$ scd scdY calculos_Tau steps calculos_QR tempoproc];

num2str(Resultados)

\subsection{Código-Fonte Andrews}

Arquivo: Problema_Andrews.m

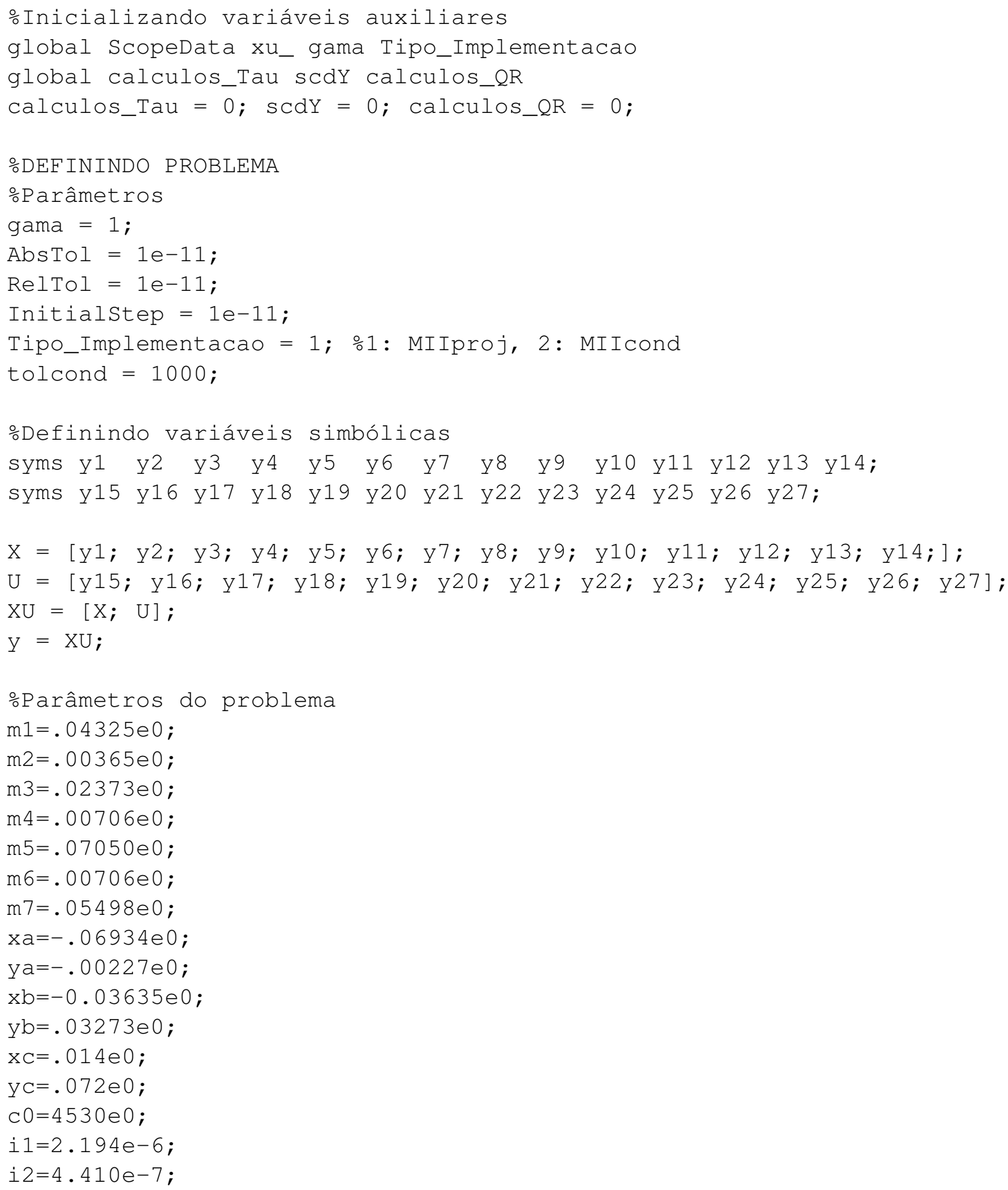




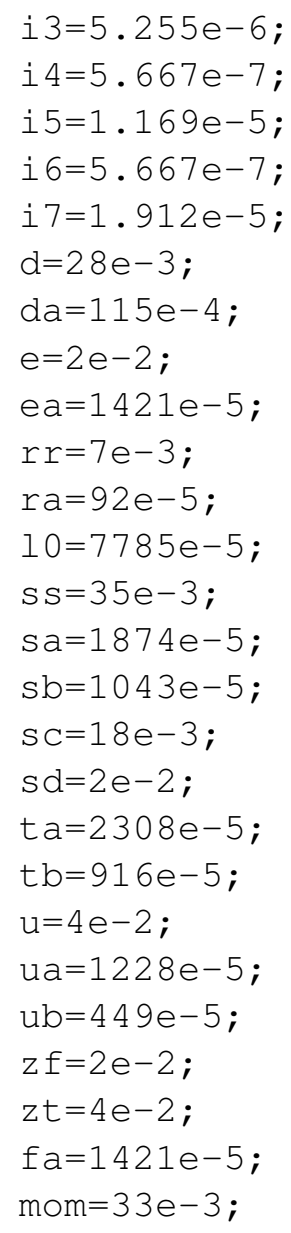




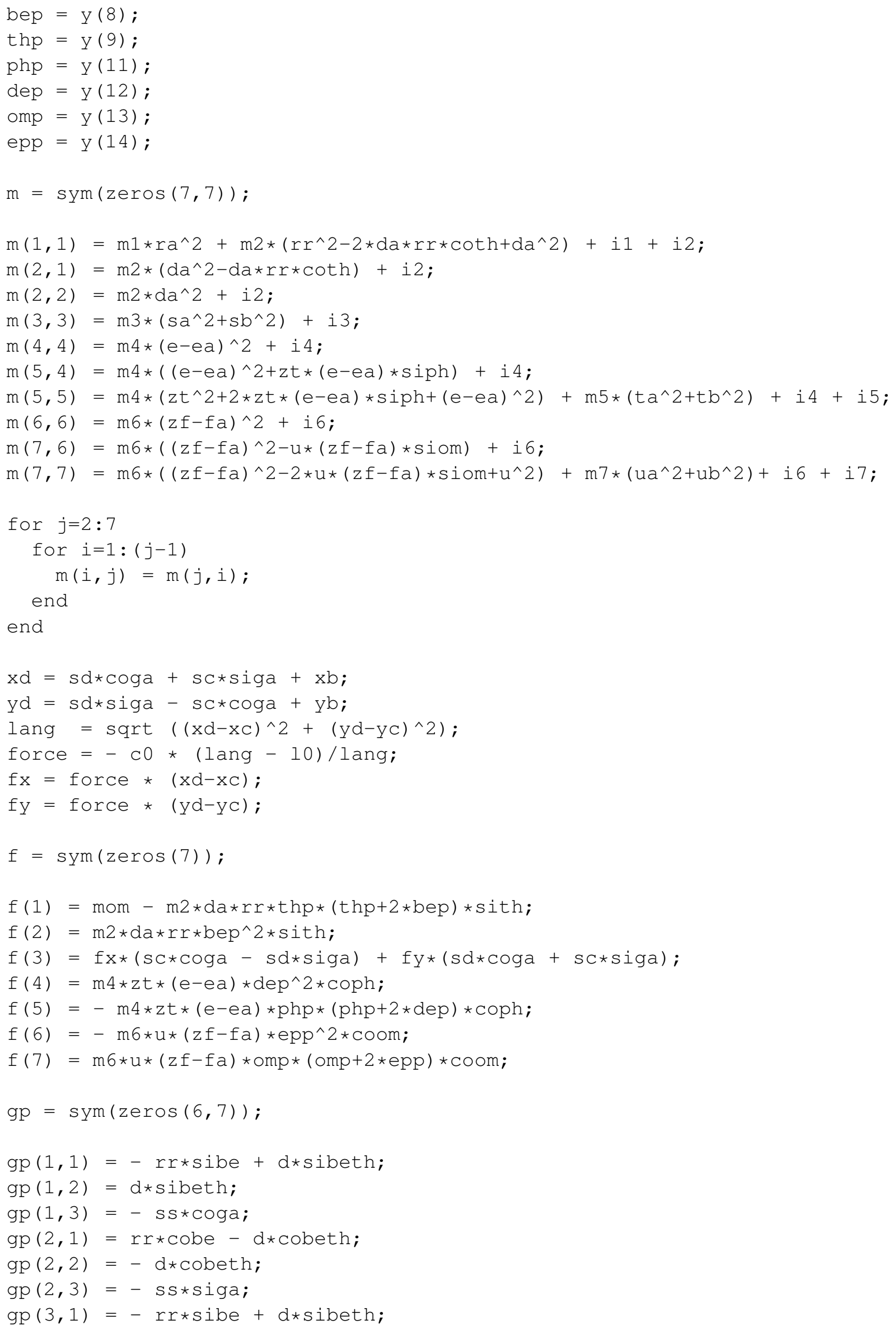




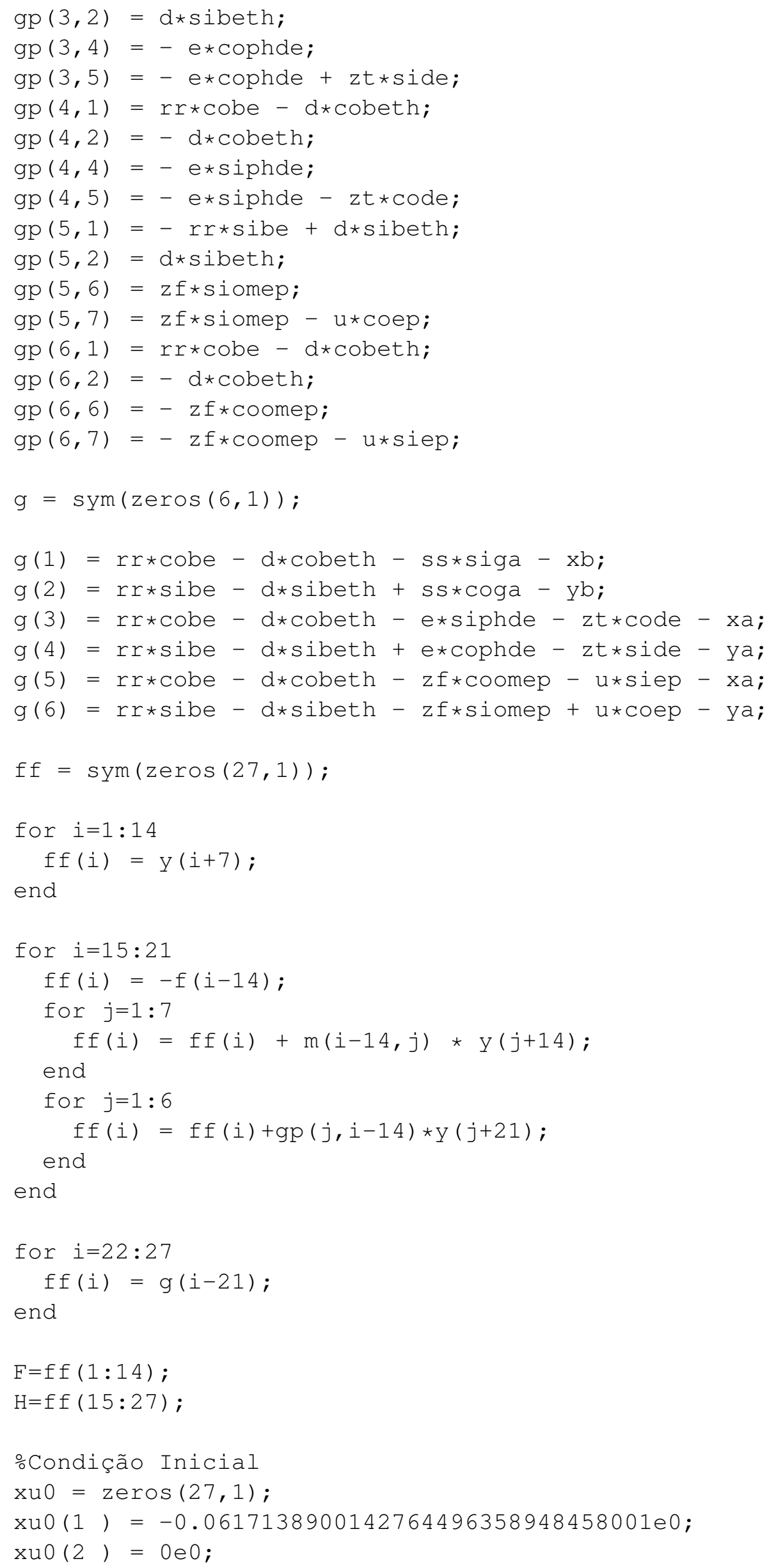


$\mathrm{xu0}(3)=0.455279819163070380255912382449 \mathrm{e} 0 ;$

$\mathrm{xu0}(4)=0.222668390165885884674473185609 \mathrm{e} 0 ;$

$\mathrm{xuO}(5)=0.487364979543842550225598953530 \mathrm{e} 0 ;$

$\operatorname{xu0}(6)=-0.222668390165885884674473185609 \mathrm{e} 0 ;$

$\mathrm{xu0}(7)=1.23054744454982119249735015568 \mathrm{e} 0 ;$

$\mathrm{xu0}(8)=0 \mathrm{e} 0$;

$\operatorname{xu0}(9)=0 e 0$;

$\operatorname{xu} 0(10)=0 e 0 ;$

$\operatorname{xuO}(11)=0 e 0 ;$

$\mathrm{xuO}(12)=0 \mathrm{e} 0$;

$\operatorname{xuO}(13)=0 e 0$;

$\mathrm{xuO}(14)=0 \mathrm{e} 0$;

$\operatorname{xu0}(15)=14222.4439199541138705911625887 e 0 ;$

$\operatorname{xu0}(16)=-10666.8329399655854029433719415 \mathrm{e} 0 ;$

$\operatorname{xu0}(17)=0 e 0$;

$\mathrm{xuO}(18)=0 \mathrm{e} 0$;

$\mathrm{xuO}(19)=0 \mathrm{e} 0$;

$\operatorname{xuO}(20)=0 e 0 ;$

$\mathrm{xu} 0(21)=0 \mathrm{e} 0$;

$\mathrm{xu0}(22)=98.5668703962410896057654982170 \mathrm{e} 0$;

$\operatorname{xu0}(23)=-6.12268834425566265503114393122 e 0$;

$\mathrm{xuO}(24)=0 \mathrm{e}$;

$\mathrm{xuO}(25)=0 \mathrm{e} 0$;

$\mathrm{xu0}(26)=0 \mathrm{e} 0$;

$\mathrm{xuO}(27)=0 \mathrm{e}$;

$\mathrm{yFimRef}=\operatorname{zeros}(27,1)$;

yFimRef $(1)=0.1581077119629904 \mathrm{e}+2$;

$\mathrm{yFimRef}(2)=-0.1575637105984298 \mathrm{e}+2$;

yFimRef $(3)=0.4082224013073101 \mathrm{e}-1$;

$\mathrm{yFimRef}(4)=-0.5347301163226948 \mathrm{e}+0$;

$\mathrm{yFimRef}(\mathrm{5})=0.5244099658805304 \mathrm{e}+0$;

$\mathrm{yFimRef}(6)=0.5347301163226948 \mathrm{e}+0$;

yFimRef $(7)=0.1048080741042263 e+1$;

yFimRef $(8)=0.1139920302151208 \mathrm{e}+4$;

$\mathrm{yFimRef}(9)=-0.1424379294994111 \mathrm{e}+4$;

$\mathrm{yFimRef}(10)=0.1103291221937134 \mathrm{e}+2$;

$\mathrm{yFimRef}(11)=0.1929337464421385 e+2$;

$\mathrm{yFimRef}(12)=0.5735699284790808 \mathrm{e}+0$;

$\mathrm{yFimRef}(13)=-0.1929337464421385 e+2$;

$\mathrm{yFimRef}(14)=0.3231791658026955 e+0$;

$y F i m R e f(15)=-0.2463176316945196 e+5$;

yFimRef $(16)=0.5185037701610329 e+5$;

yFimRef $(17)=0.3241025686413781 e+6$;

yFimRef $(18)=0.5667493645176213 e+6$;

$\mathrm{yFimRef}(19)=0.1674362929479361 \mathrm{e}+5$;

$\mathrm{yFimRef}(20)=-0.5667493645176222 \mathrm{e}+6$;

$\mathrm{yFimRef}(21)=0.9826520791458422 \mathrm{e}+4$;

yFimRef $(22)=0.1991753333731910 e+3$;

$\mathrm{yFimRef}(23)=-0.2975531228015052 e+2$;

yFimRef $(24)=0.2306654119098399 e+2$;

yFimRef $(25)=0.3145271365475927 e+2$; 


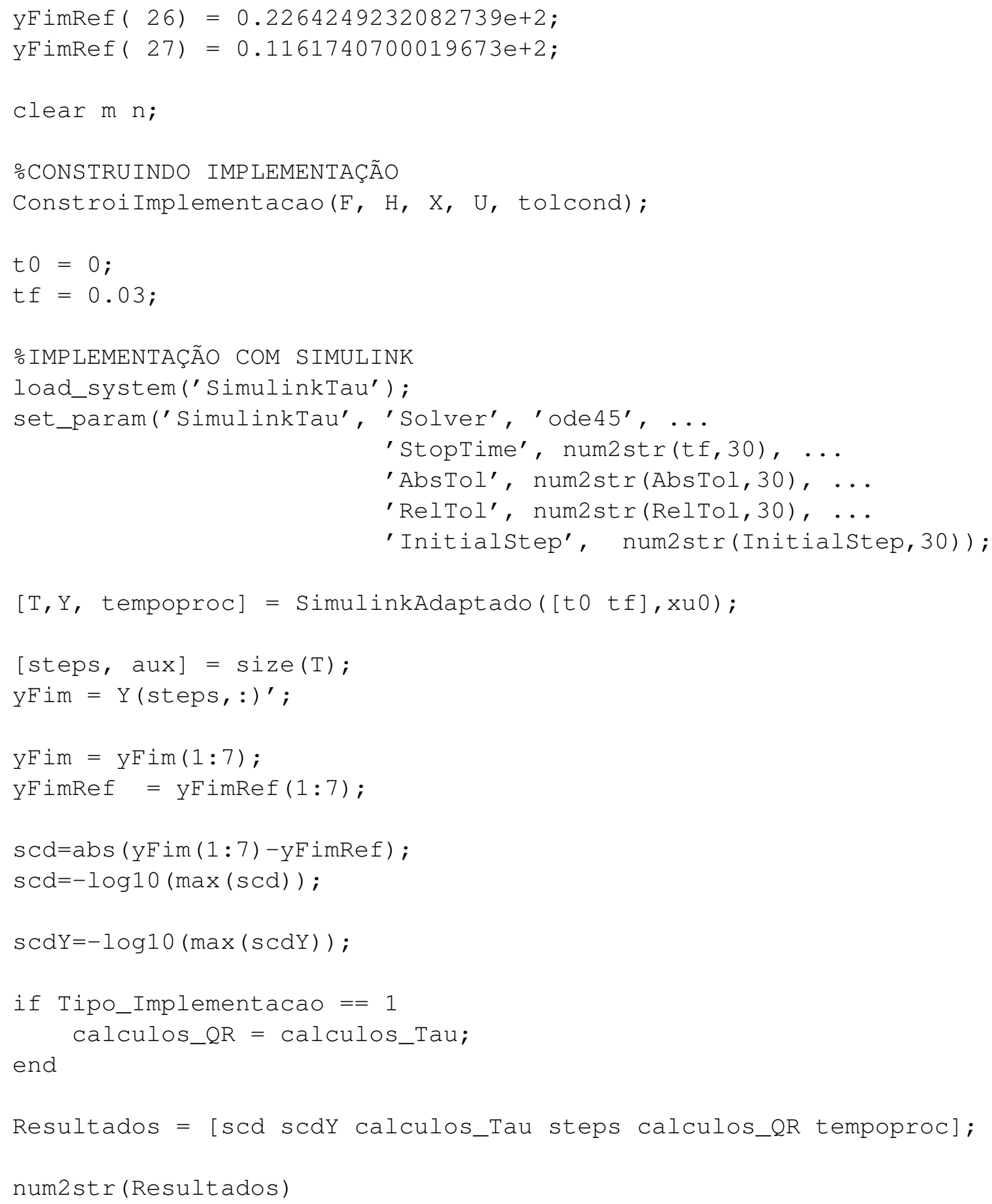

\subsection{Código-Fonte TransAmp}

Arquivo: Problema_TransAmp.m

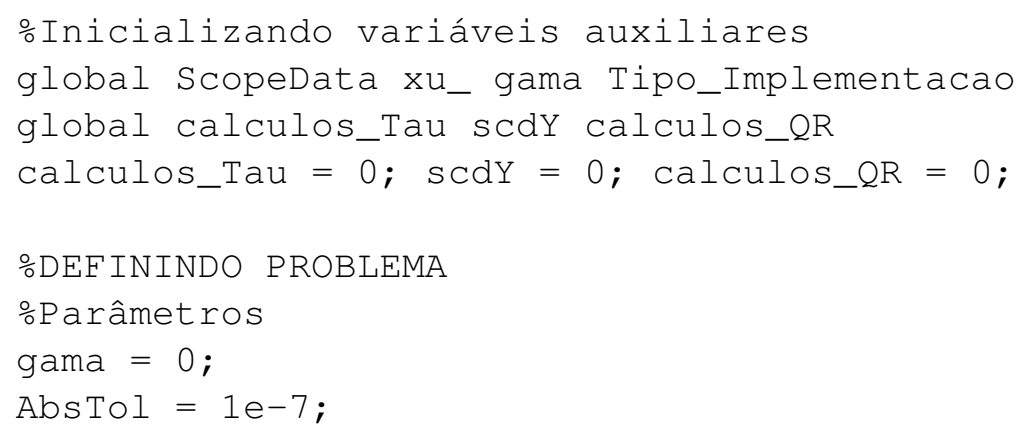




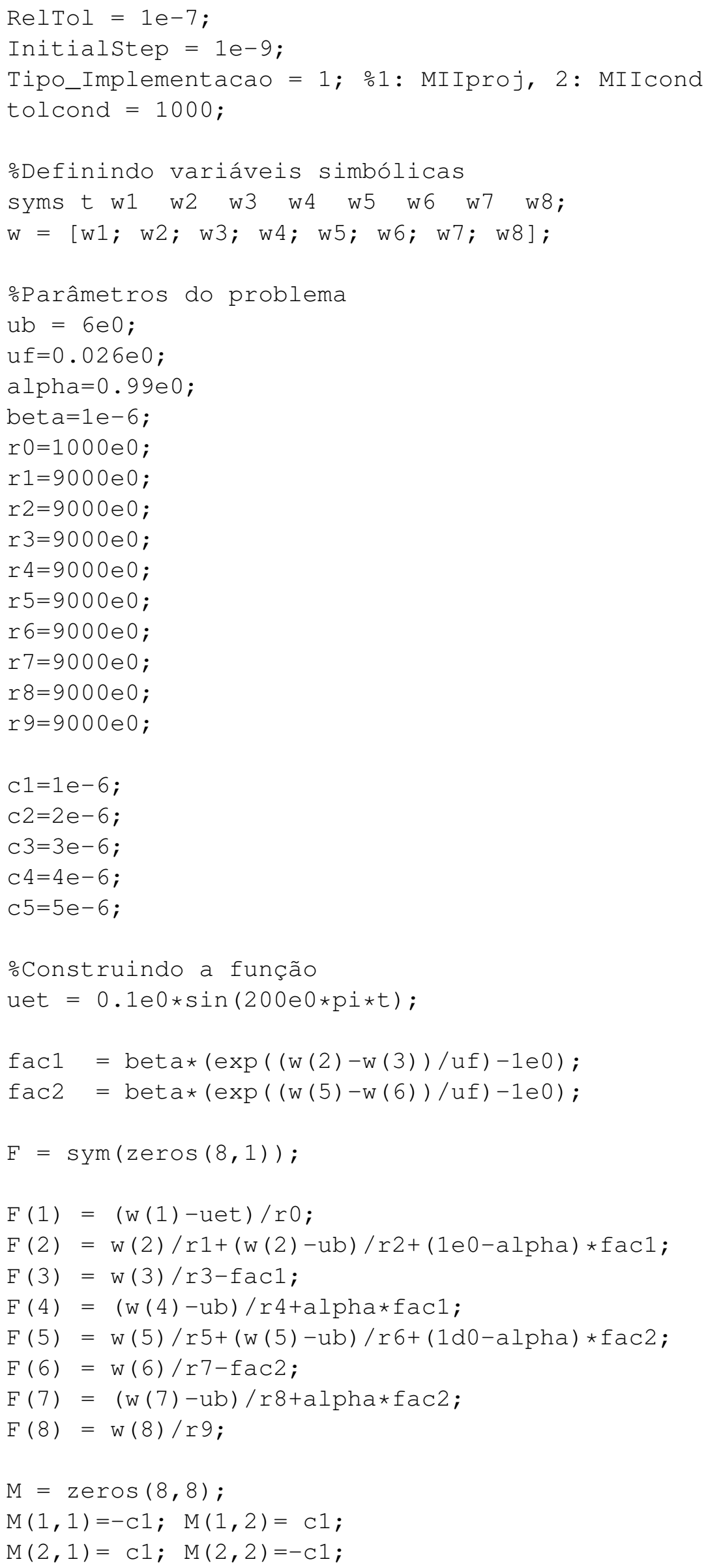




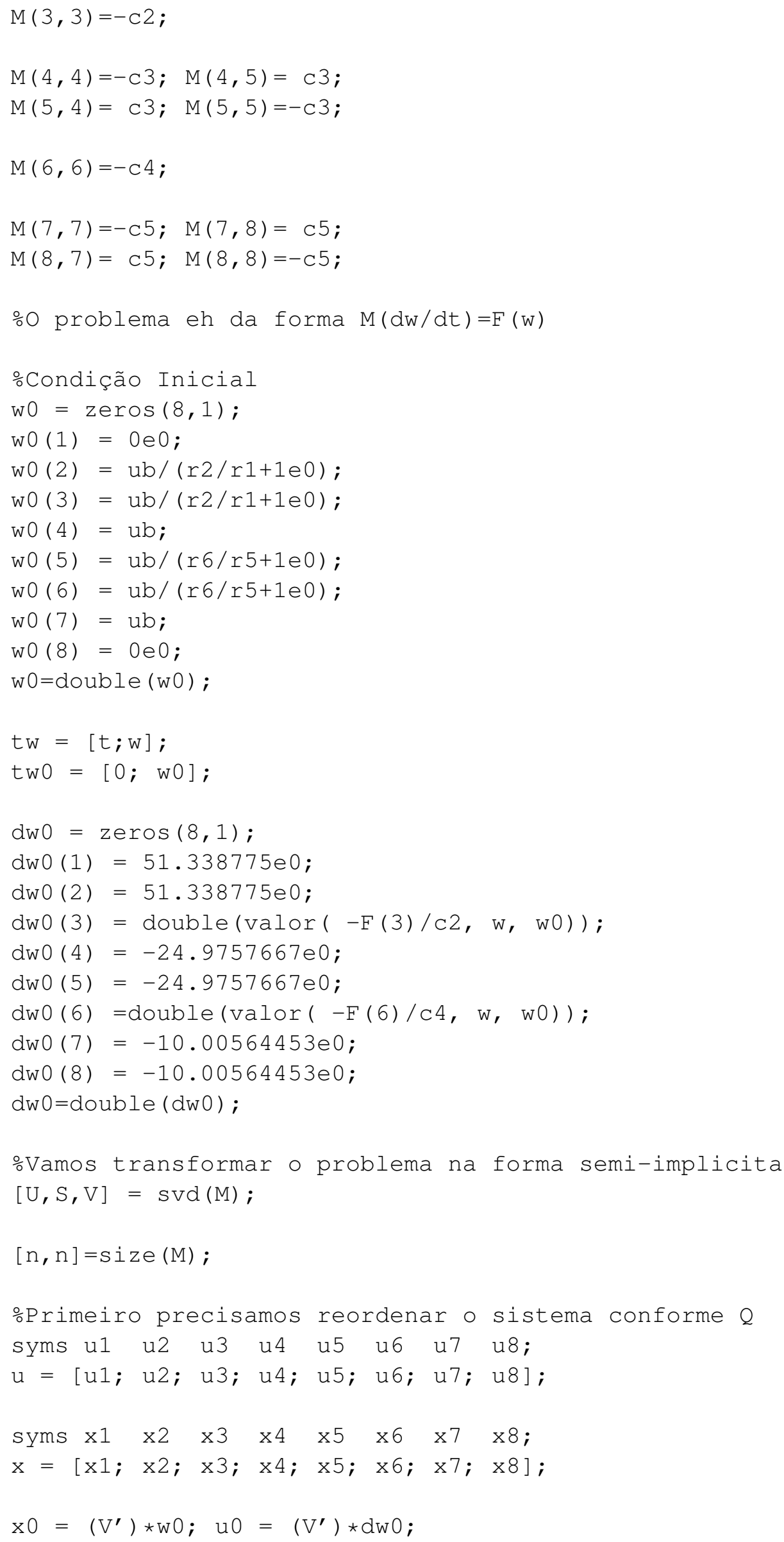


$t x=[t ; x] ; t x 0=[0 ; x 0] ;$

Ftil $=\left(U^{\prime}\right) * \operatorname{valor}(F, W, V * x)$;

$\mathrm{n}=$ length $(\mathrm{w}) ; \mathrm{r}=5$;

$\mathrm{h}=\operatorname{Ftil}((\mathrm{r}+1): \mathrm{n})$;

Ftil = Ftil $(1: r)$;

oInvertendo a matriz diagonal

$S=S(1: r, 1: r) ;$ invS = eye $(r)$;

for $i=1: r$ $\operatorname{invS}(i, i)=1 / S(i, i)$;

end

$\mathrm{u}=\mathrm{u}((\mathrm{r}+1): \mathrm{n})$;

$\mathrm{uO}=\mathrm{uO}((\mathrm{r}+1): \mathrm{n})$;

$f=[i n v S * F t i l ; u]$

$\mathrm{xu}=[\mathrm{x} ; \mathrm{u}]$;

$\mathrm{xuO}=[\mathrm{xO} ; \mathrm{uO}]$;

yFimRef $=\operatorname{zeros}(\mathrm{n}, 1)$;

yFimRef $(1)=-0.5562145012262709 e-002$;

$y F i m R e f(2)=0.3006522471903042 e+001$;

yFimRef $(3)=0.2849958788608128 e+001$;

$\mathrm{yFimRef}(4)=0.2926422536206241 \mathrm{e}+001$;

$\mathrm{yFimRef}(5)=0.2704617865010554 \mathrm{e}+001$;

$\mathrm{yFimRef}(6)=0.2761837778393145 e+001$;

$\mathrm{yFimRef}(7)=0.4770927631616772 e+001$;

yFimRef $(8)=0.1236995868091548 e+001$;

○CONSTRUINDO IMPLEMENTAÇÃO

ConstroiImplementacao(f, $h, t x, u$, tolcond);

to $=0$;

$\mathrm{tf}=0.2$;

load_system('SimulinkTau');

set_param('SimulinkTau', 'Solver', 'ode45', ...

'StopTime', num2str(tf,30), ...

'AbsTol', num2str(AbsTol,30), ...

'RelTol', num2str(RelTol,30), ...

'Initialstep', num2str(Initialstep, 30));

$[\mathrm{T}, \mathrm{Y}$, tempoproc $]=$ SimulinkAdaptado $\left(\left[\begin{array}{ll}\mathrm{t} & \mathrm{tf}\end{array}\right], \mathrm{xu} 0\right) ;$

[steps, aux] = size $(\mathrm{T})$;

$\mathrm{yFim}=\mathrm{Y}(\text { steps, : })^{\prime}$; 


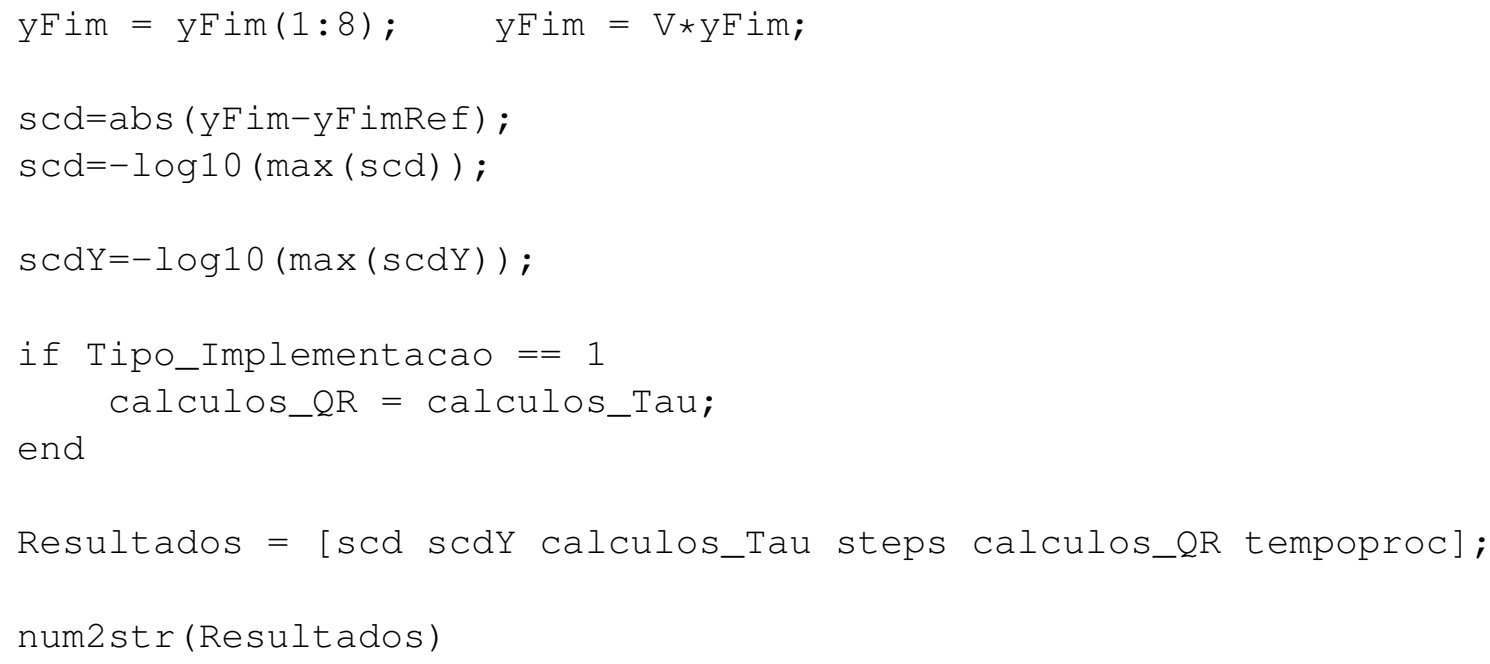

\subsection{Código-Fonte CarAxis}

Arquivo: Problema_CarAxis.m

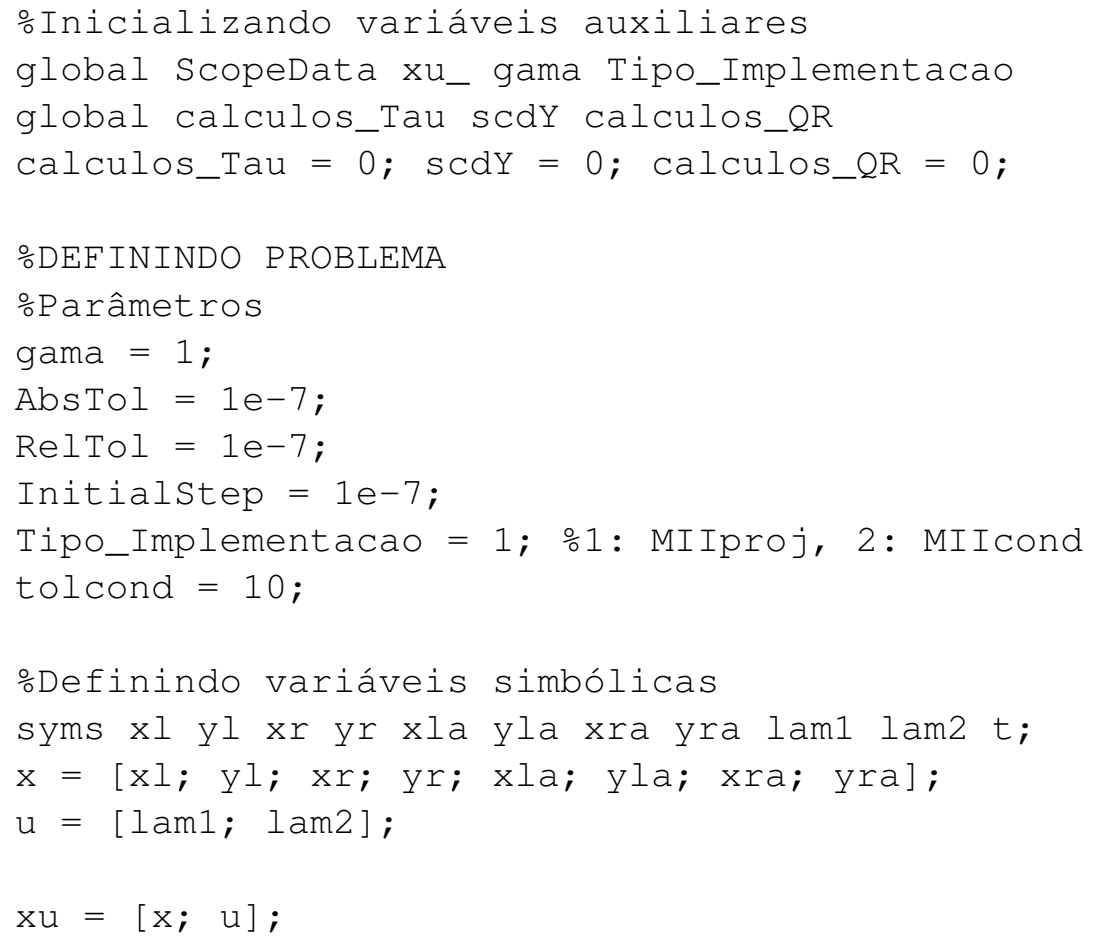




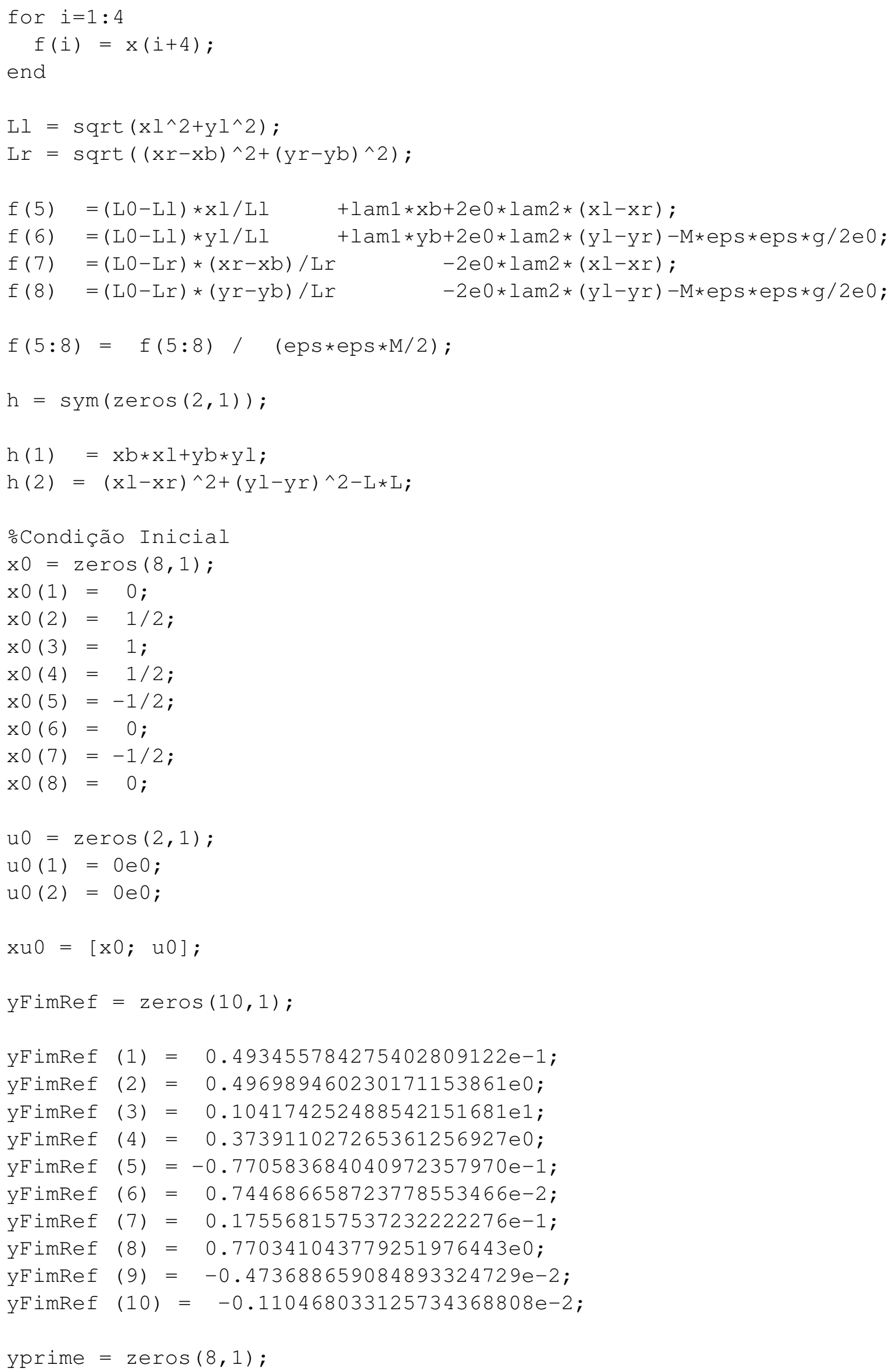




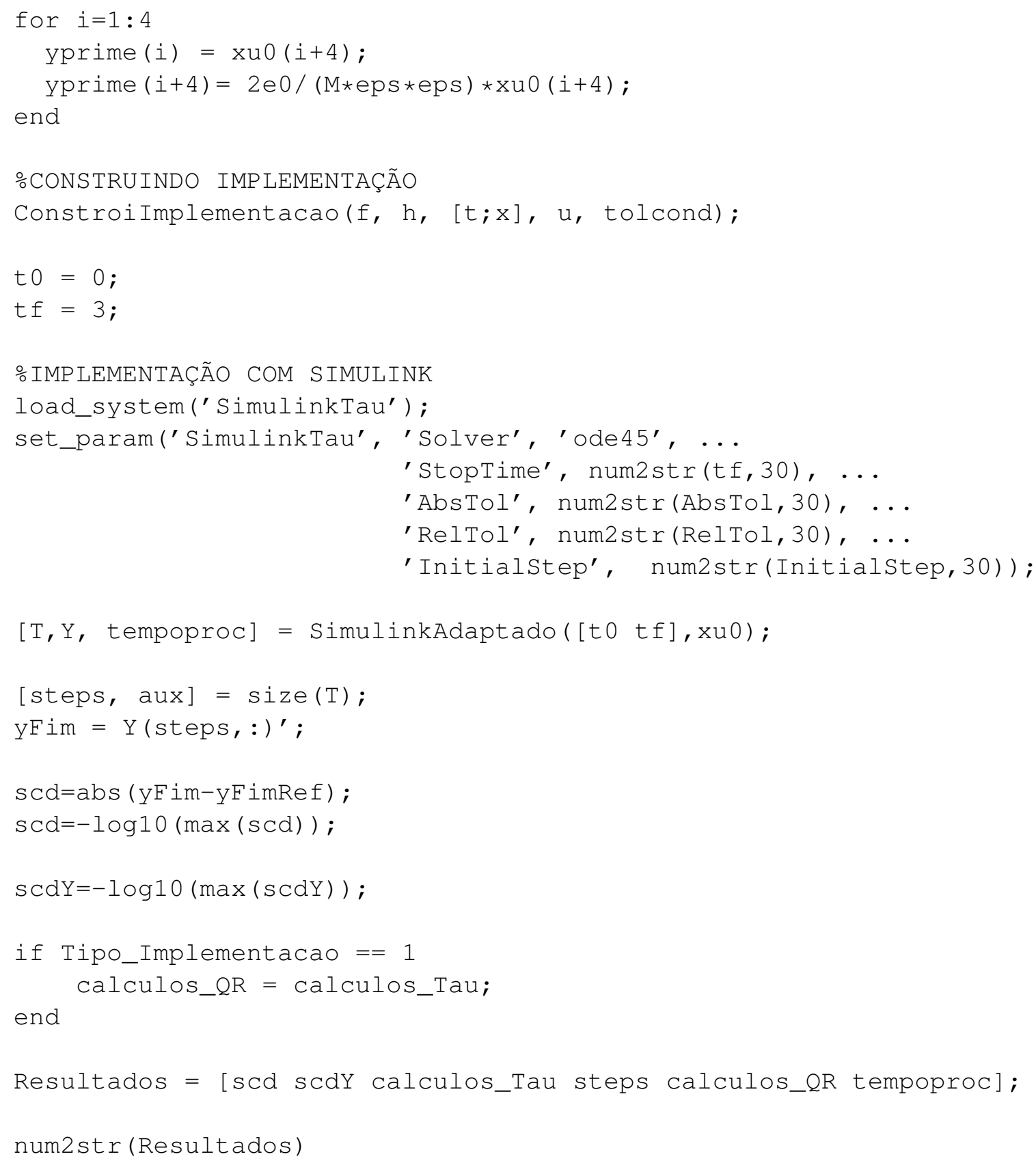




\section{Referências Bibliográficas}

[AP98] U. M. Ascher e L. R. Petzold. Computer methods for ordinary differential equations and differential-algebraic equations. Society for Industrial and Applied Mathematics (SIAM). Philadelphia, PA, 1998. 1, 48, 55

[BCP96] K. E. Brenan, S. L. Campbell, and L. R. Petzold. Numerical Solution of Initial-Value Problems in Differential-Algebraic Equations. Society for Industrial and Applied Mathematics - (SIAM). second edition, 1996. 1

[BMM06] L. Brugnano, C. Magherini, and F. Mugnai. Blended implicit methods for the numerical solution of dae problems.. J. Comput. Appl. Math,. 189:34-50, 2006. 1

[Cam82] S. L. Campbell. Singular Systems of Differential Equations. Pitman, London. 1982. 1

[Cam90] S. L. Campbell. Descriptor system in the 90's. In Proc. 29th IEEE Conf. Dec. Control. pages, 442-447, 1990. 1

[Cas03] J. Cash. Efficient numerical methods for the solution of stiff initial-value problems and differential algebraic equations. Proc. Roy. Soc. London, A. 459:797 815, 2003. 1

[Dai89] Dai Liyi. Singular Control System. Springer-Verlag. 1989. 1

[FN02] Djairo Figueiredo e A. F. Neves. Equações Diferenciais Aplicadas. 2a ed. IMPA,2002, 301p. 48,52

[Fre07] Celso B. N. Freitas. Integração numérica de sistemas não lineares semi-implícitos via teoria de controle geométrico. Trabalho de conclusão de curso, IME-USP, 2007. 7, 22

[HLR89] E. Hairer, C. Lubich, and M. Roche. The Numerical Solution of Differential-Algebraic Systems by Runge-Kutta Methods. Lecture Notes in Mathematics 1409. SpringerVerlag, 1989. 1, 48

[HW99] E. Hairer and G. Wanner. Stiff differential equations solved by radau methods. Appl. Math., 111:93-111, 1999. 1997. 1

[HW96] E. Hairer and G. Wanner.. Solving Ordinary Differential Equations II: Stiff and Differential-algebraic Problems. Springer-Verlag, second revised edition, 1996. 1, 48

[IM98] F. Iavernaro and F. Mazzia. Solving ordinary differential equations by generalized adams methods: properties and implementation techniques. Appl. Num. Math. 28:107126, 1998. 1

[KD99] A. Kumar e P. Daoutidis. Control of nonlinear differential algebraic equation systems. Chapman \& Hall/CRC, Boca Raton, FL, 1999. 1

[MM08] Francesca Mazzia e Cecilia Magherini. Test Set for Initial Value Problem Solvers, release 2.4. 2008, February. Department of Mathematics, University of Bari and INdAM, Research Unit of Bari. Disponível em http://www.dm.uniba.it/ testset. 1, $2,45,46,47,48,58,59,68$ 
[NS90] H. Nijmeijer e A. J. Van der Shaft. Nonlinear dynamical control. New York: SpringerVerlag, 1990. 7, 10, 14

[Pon62] L. Pontrjagin. Ordinary Differential Equations.. Addison Wesley, 1962. 29

[Sch94] S. Schneider. Intégration de systèmes d'équations différentielles raides et différentielles-algébriques par des méthodes de collocations et méthodes générales linéaires. PhD thesis, Université de Genève, 1994. 1, 48

[SDS05] Paulo S. P. da Silva, Emmanuel Delaleau e Iderval S. de Souza. On geometric control and numeric integration of DAE's. In: IFAC World Congress 05, 2005, Praga, 2005, v.1, p-16. $7,22,48$

[SLV97] J. J. B. de Swart, W. M. Lioen, and W. A. van der Veen. Specification of PSIDE. CWI. 1997. 1

[Sot79] J. Sotomayor Lições de Equações Diferenciais Ordinárias. Projeto Euclides, 1979. $10,18,21$

[Sou06] Iderval S. de Souza. Geometria do desacoplamento e integração numérica de equações diferenciais não lineares implícitas. Dissertação de Mestrado, Escola Politécnica, 2006. 7,22

[Sto98] W. J. H. de Stortelder. Parameter Estimation in Nonlinear Dynamical Systems. PhD thesis, University of Amsterdam, March 12, 1998. 1, 48

[Str80] G. Strang. Linear algebra and its applications. 2nd ed. New York: Academic. Press, 1980. 33, 36, 39

[SW02] Paulo S. P. da Silva e Carlos J. Watanabe. Some Geometric Properties of DifferentialAlgebraic Equation. Relatório interno, disponível em http://www.lac.usp.br/. 7 